\title{
Round-Trip Oxidative Addition, Ligand Metathesis, and Reductive Elimination in a PIII/PV Synthetic Cycle
}

\author{
Soohyun Lim and Alexander T. Radosevich* \\ Department of Chemistry, Massachusetts Institute of Technology, Cambridge, MA 02139, United States \\ Correspondence to: radosevich@mit.edu
}

\section{Contents}

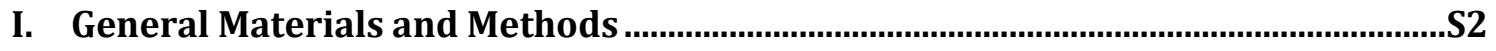

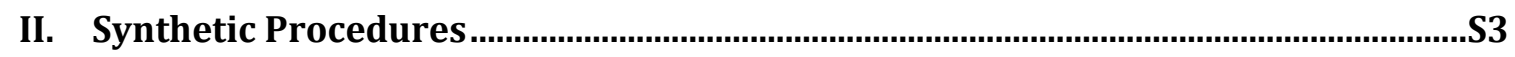

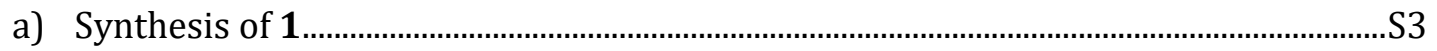

b) Synthesis of 1•[F][C $\left.\mathrm{C}_{5} \mathrm{~F}_{4} \mathrm{E}\right]$ via C-F Oxidative Addition ..................................................S

c) Preparation of Organoaluminum Reagents ......................................................................S6

d) Synthesis of 1•[H] $\left[\mathrm{C}_{5} \mathrm{~F}_{4} \mathrm{E}\right]$ via Ligand Metathesis ............................................................ 6

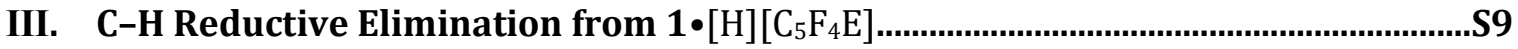

a) General Procedure for C-H Reductive Elimination ...........................................................

b) Hydrodefluorination Product Assignment ...........................................................................S12

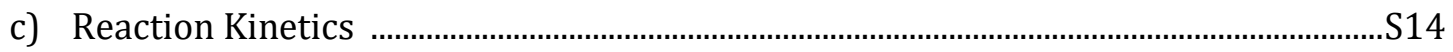

d) One-Pot Ligand Metathesis and Reductive Elimination .................................................. 15

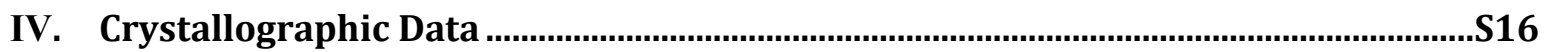

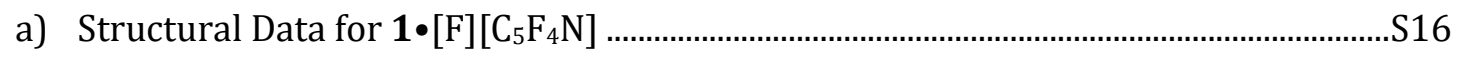

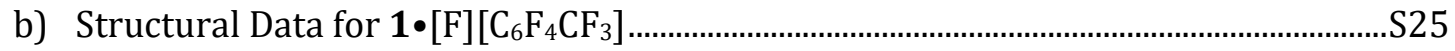

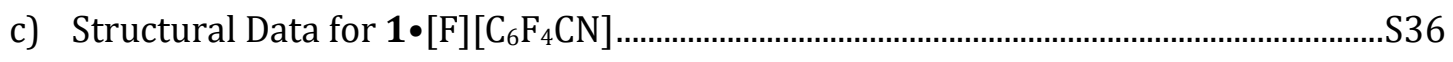

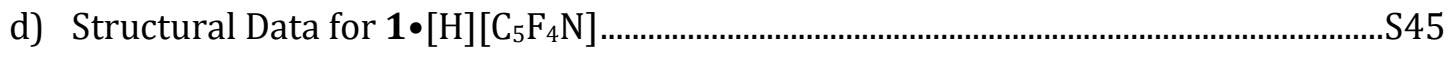

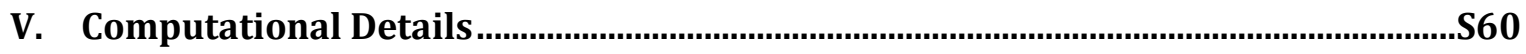

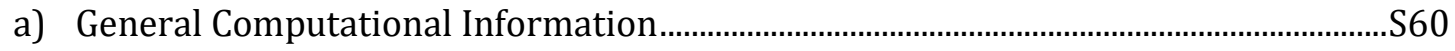

b) Reaction Mechanism for Reductive Elimination...............................................................S60

c) Energies and Cartesian Coordinates of Stationary Points ..............................................S64

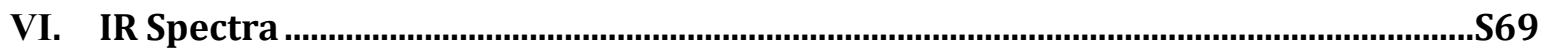

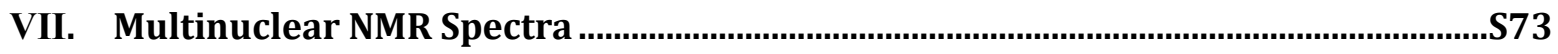

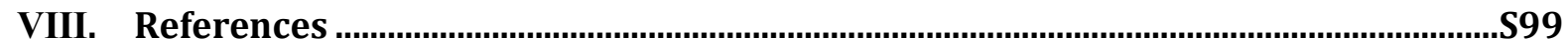




\section{General Materials and Methods}

All reagents were purchased from commercial vendors (Sigma-Aldrich, Alfa Aesar, Acros, TCI, Oakwood Chemical, or Combi-Blocks) and used without further purification. Toluene, pentane, diethyl ether, dichloromethane, and tetrahydrofuran were purified and collected under argon using a Glass Contour Solvent Purification System, and further dried with $3 \AA$ or $4 \AA$ molecular sieves. Anhydrous benzene was obtained from a Sigma-Aldrich (sure-seal ${ }^{\circledR}$ bottle) and dried with $3 \AA$ molecular sieves. All glassware was oven-dried at $120{ }^{\circ} \mathrm{C}$ prior to use. ${ }^{1} \mathrm{H},{ }^{13} \mathrm{C}\left\{{ }^{1} \mathrm{H}\right\},{ }^{19} \mathrm{~F},{ }^{19} \mathrm{~F}\left\{{ }^{1} \mathrm{H}\right\},{ }^{31} \mathrm{P}$, and ${ }^{31} \mathrm{P}\left\{{ }^{1} \mathrm{H}\right\}$ spectra were recorded with either a Bruker Advance Neo 400, a Bruker Advance Neo 500, or a JEOL ECZ 500 spectrometers and processed using MestReNova. ${ }^{1}{ }^{1} \mathrm{H}$ NMR chemical shifts are given in ppm with respect to a solvent residual peak $\left(\mathrm{CDCl}_{3}, \delta 7.26 \mathrm{ppm}\right.$; toluene- $d_{8}, \delta 2.08 \mathrm{ppm} ; \mathrm{C}_{6} \mathrm{D}_{6}, \delta$ $7.16 \mathrm{ppm}) .{ }^{13} \mathrm{C}\left\{{ }^{1} \mathrm{H}\right\} \mathrm{NMR}$ shifts are given in ppm with respect to a solvent residual peak $\left(\mathrm{CDCl}_{3}, \delta 77.16\right.$ ppm). ${ }^{19} \mathrm{~F}$ and ${ }^{19} \mathrm{~F}\left\{{ }^{1} \mathrm{H}\right\}$ NMR shifts are given in ppm with respect to $\mathrm{CFCl}_{3}(\delta 0.0 \mathrm{ppm})$ as an external standard. ${ }^{31} \mathrm{P}$ and ${ }^{31} \mathrm{P}\left\{{ }^{1} \mathrm{H}\right\}$ NMR shifts are given in ppm with respect to $85 \% \mathrm{H}_{3} \mathrm{PO}_{4}(\delta 0.0 \mathrm{ppm})$ as an external standard. Multiplicities are described as s = singlet, $\mathrm{d}=$ doublet, $\mathrm{dd}=$ doublet of doublets, $\mathrm{ddd}=$ doublet of doublets of doublets, $\mathrm{dt}=$ doublet of triplets, $\mathrm{dm}=$ doublet of multiplets, $\mathrm{t}=$ triplet, $\mathrm{td}=$ triplet of doublets, $\mathrm{tm}=$ triplet of multiplets, $\mathrm{q}=$ quartet, $\mathrm{m}=$ multiplet. Coupling constants are reported in Hertz (Hz). FTIR spectra were collected with a Bruker Alpha II FTIR spectrometer with a Diamond Crystal ATR accessory. High-resolution mass spectra were obtained using a JEOL AccuTOF 4G LC-plus equipped with an ionSense DART (Direct Analysis in Real Time) source. X-ray diffraction data of $1 \cdot[\mathrm{F}]\left[\mathrm{C}_{5} \mathrm{~F}_{4} \mathrm{~N}\right]$ was collected on a Bruker SMART APEX CCD area detector system equipped with a graphite monochromator and a MoK $\alpha$ fine-focus sealed tube $(\lambda=0.71073 \AA)$. The other X-ray diffraction data were collected using $0.24797 \AA\left(\mathbf{1} \cdot[\mathrm{F}]\left[\mathrm{C}_{6} \mathrm{~F}_{4} \mathrm{CF}_{3}\right]\right)$ or $0.41328 \AA\left(\mathbf{1} \cdot[\mathrm{F}]\left[\mathrm{C}_{6} \mathrm{~F}_{4} \mathrm{CN}\right]\right.$ and 1•[H] $\left[\mathrm{C}_{5} \mathrm{~F}_{4} \mathrm{~N}\right]$ ) radiation at temperature of $15 \mathrm{~K}$ (Oxford Diffraction Helijet) on a vertical mounted Bruker D8 three-circle platform goniometer equipped with an Apex II CCD at ChemMatCARS located at Advances Photon Source (APS), Argonne National Laboratory (ANL). Raw data integration and reduction were performed with $\mathrm{SAINT}^{2}$ and $\mathrm{SADABS}^{3}$ programs. Structures were solved using SHELXT $^{4}$ and refined using least-squares methods on $\mathrm{F}^{2} \mathrm{using}$ the SHELXL software package. All nonhydrogen atoms were refined with anisotropic displacement parameters. The hydrogen atom bound to the phosphorus atom of $\mathbf{1} \cdot[\mathrm{H}]\left[\mathrm{C}_{5} \mathrm{~F}_{4} \mathrm{~N}\right]$ was located in Fourier maps and was refined isotropically without constraints. All other hydrogen atoms were fixed in their ideal geometries and refined isotropically. Graphical representation was done using the XP program. 


\section{Synthetic Procedures}

\section{a) Synthesis of 1}

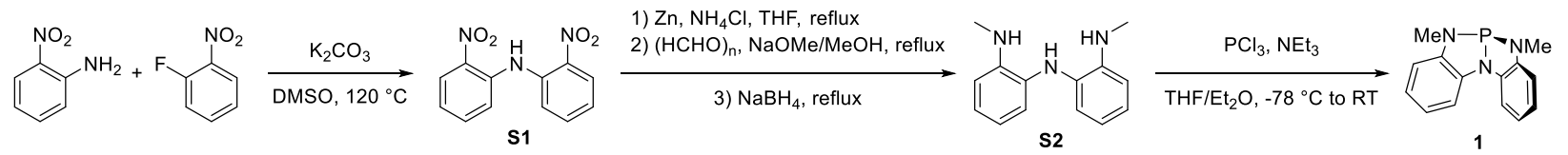

Bis(2-nitrophenyl)amine (S1): Synthesized according to a modified literature procedure. ${ }^{5}$ To a $1 \mathrm{~L}$ round bottom flask containing a stir bar 1-Fluoro-2-nitrobenzene (12.0 mL, $114 \mathrm{mmol}, 1$ equiv), 2nitroaniline (15.7 g, $114 \mathrm{mmol}, 1.0$ equiv), potassium carbonate (18.9 g, $137 \mathrm{mmol}, 1.2 \mathrm{equiv}$ ) and dimethyl sulfoxide $(300 \mathrm{~mL})$ were added. The reaction mixture was stirred at $120{ }^{\circ} \mathrm{C}$ for $48 \mathrm{~h}$. Water $(150 \mathrm{~mL})$ was then added and the mixture was extracted with dichloromethane $(3 \times 250 \mathrm{~mL})$. The combined organic layer was washed with a saturated aqueous solution of sodium chloride $44 \times 100$ $\mathrm{mL}, 15 \%$ ), dried over anhydrous sodium sulfate and concentrated in vacuo to afford an orange solid (27.4 g, $106 \mathrm{mmol}, 92.9 \%)$. The product was used in the next step without further purification. ${ }^{1} \mathrm{H}$ $\operatorname{NMR}\left(500 \mathrm{MHz}, \mathrm{CDCl}_{3}\right.$ ): $\delta 11.01(\mathrm{~s}, 1 \mathrm{H}), 8.21$ (dd, $\left.J=8.5,1.6 \mathrm{~Hz}, 2 \mathrm{H}\right), 7.59$ (dd, J = 8.5, $1.4 \mathrm{~Hz}, 2 \mathrm{H}$ ), 7.53 $(\mathrm{ddd}, J=8.5,7.0,1.6 \mathrm{~Hz}, 2 \mathrm{H}), 7.10(\mathrm{ddd}, J=8.5,7.0,1.4 \mathrm{~Hz}, 2 \mathrm{H}) \mathrm{ppm}$.

Bis(2-methylaminophenyl)amine (S2): Synthesized according to modified literature procedures.6,7 To a $500 \mathrm{~mL}$ round bottom flask containing a stir bar were added $\mathbf{S 1}$ (7.50 g, $28.9 \mathrm{mmol}, 1$ equiv), zinc dust (26.5 g, $405 \mathrm{mmol}, 14.0$ equiv), and ammonium chloride (20.3 g, $380 \mathrm{mmol}, 13.1 \mathrm{equiv}$ ). The flask was equipped with a reflux condenser and connected to Schlenk line. The atmosphere was exchanged for nitrogen via three evacuation/backfill cycles. Tetrahydrofuran (300 mL) was charged via a syringe. The suspension was heated to reflux and stirred overnight until the orange color disappeared. The reaction mixture was cooled down to ambient temperature, filtered over celite, and the filtrate was dried in vacuo. To the dried filtrate were added a stir bar, paraformaldehyde $(8.57 \mathrm{~g}$, $285 \mathrm{mmol}, 9.86$ equiv) and methanol (300 mL). The suspension was cooled down in an ice/water bath, and a solution of sodium methoxide ( $25 \%$ in methanol, $20 \mathrm{~mL}$ ) was added slowly. The reaction mixture was then stirred under reflux for 1 hour. The reaction mixture was cooled to ambient temperature and placed in an ice/water bath. Sodium borohydride (11.9 g, $314 \mathrm{mmol}, 10.8$ equiv) was added in small portions, and the mixture was again stirred under reflux for 1 hour. After being cooled to ambient temperature, an aqueous solution of sodium hydroxide (1 M, $150 \mathrm{~mL}$ ) was added to precipitate a solid. The flask was kept in a freezer overnight. The next day, the solid was filtered, washed with water, and dried under Schlenk line vacuum at $40{ }^{\circ} \mathrm{C}$ overnight to yield the product as a purple solid (4.86 g, $21.4 \mathrm{mmol}, 73.9 \%$ ). ${ }^{1} \mathrm{H} \mathrm{NMR}\left(500 \mathrm{MHz}, \mathrm{CDCl}_{3}\right.$ ): $\delta 7.03$ (ddd, $J=8.4,6.4,2.4 \mathrm{~Hz}$, $2 \mathrm{H}), 6.74-6.67(\mathrm{~m}, 6 \mathrm{H}), 4.86(\mathrm{~s}, 1 \mathrm{H}), 3.74(\mathrm{~s}, 2 \mathrm{H}), 2.87(\mathrm{~s}, 6 \mathrm{H}) \mathrm{ppm}$. 
1: Synthesized according to a literature procedure. ${ }^{7}$ To a $250 \mathrm{~mL}$ round bottom flask containing a stir bar was added S2 (4.86 g, $21.4 \mathrm{mmol}, 1$ equiv). The flask was connected to Schlenk line and the atmosphere was exchanged for nitrogen via three evacuation/backfill cycles. Tetrahydrofuran (30 $\mathrm{mL})$ and diethyl ether $(90 \mathrm{~mL})$ were charged via syringes. To the solution cooled in a dry ice/acetone bath, phosphorus trichloride (1.87 mL, $21.4 \mathrm{mmol}, 1.0$ equiv) and triethylamine $(9.1 \mathrm{~mL}, 65 \mathrm{mmol}$, 3.1 equiv) were added dropwise via syringes. The reaction mixture was stirred at $-78{ }^{\circ} \mathrm{C}$ for 1 hour and warmed to room temperature for additional stirring for 4 hours. The volatiles were removed in vacuo and the resultant solid was brought into a nitrogen filled glove box. Pentane (150 $\mathrm{mL})$ was added to dissolve the product, and the mixture was filtered through celite. The filtrate was then dried in vacuo. The resultant solid was dissolved in minimal diethyl ether and stored in a freezer overnight to precipitate the product as colorless crystals $(2.5 \mathrm{~g}, 9.8 \mathrm{mmol}, 46 \%) .{ }^{1} \mathrm{H}$ NMR $\left(400 \mathrm{MHz}, \mathrm{CDCl}_{3}\right): \delta$ $7.43(\mathrm{dd}, J=7.8,1.3 \mathrm{~Hz}, 2 \mathrm{H}), 7.02(\mathrm{td}, J=7.6,1.3 \mathrm{~Hz}, 2 \mathrm{H}), 6.84(\mathrm{td}, J=7.6,1.3 \mathrm{~Hz}, 2 \mathrm{H}), 6.61(\mathrm{~d}, J=7.7$ $\mathrm{Hz}, 2 \mathrm{H}), 3.09$ (d, J = 8.4 Hz, 6H) ppm. ${ }^{31} \mathrm{P}\left\{{ }^{1} \mathrm{H}\right\} \mathrm{NMR}\left(162 \mathrm{MHz}, \mathrm{CDCl}_{3}\right): \delta 159.82$ (s) ppm.

\section{b) Synthesis of $1 \cdot[\mathrm{F}]\left[\mathrm{C}_{5} \mathrm{~F}_{4} \mathrm{E}\right]$ via C-F Oxidative Addition}
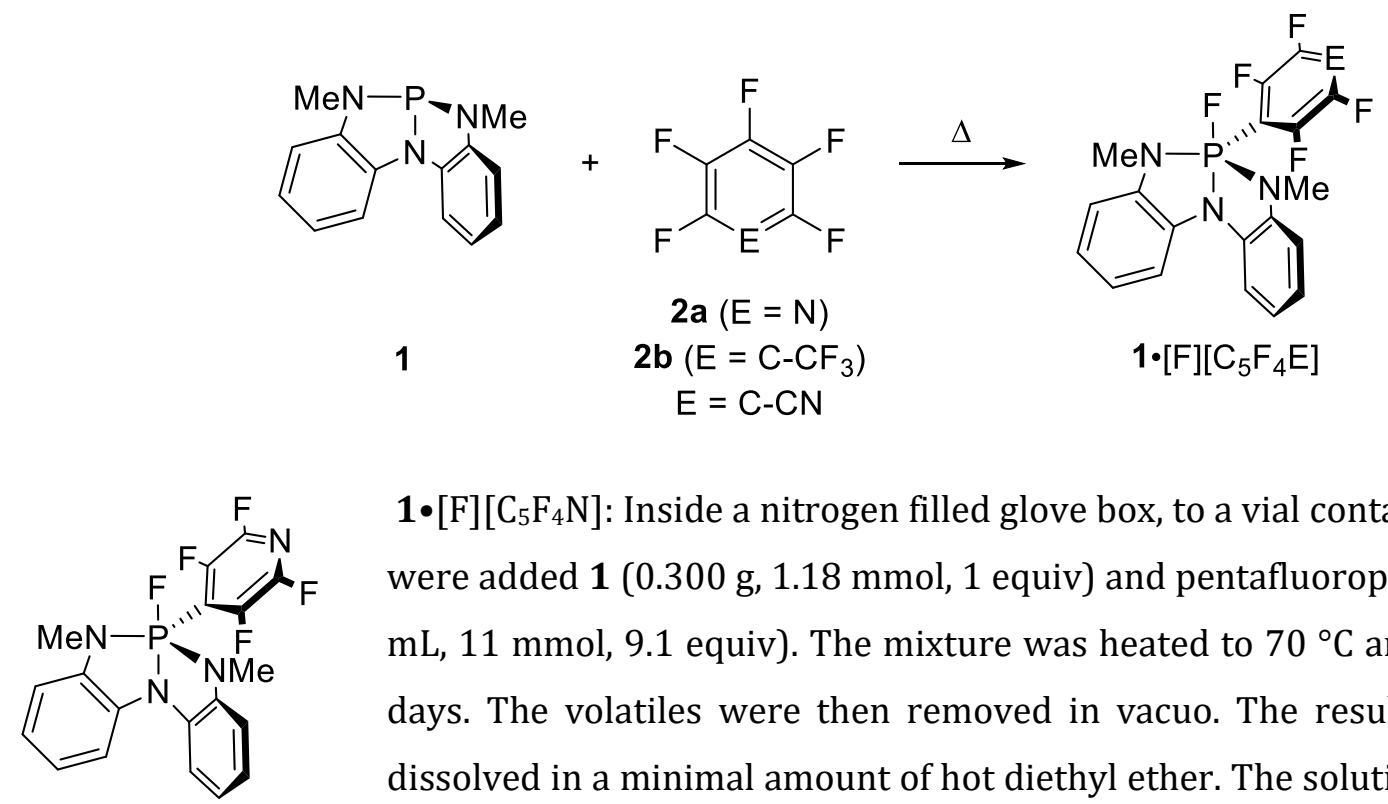

1• $[\mathrm{F}]\left[\mathrm{C}_{5} \mathrm{~F}_{4} \mathrm{~N}\right]$ : Inside a nitrogen filled glove box, to a vial containing a stir bar were added 1 ( $0.300 \mathrm{~g}, 1.18 \mathrm{mmol}, 1$ equiv) and pentafluoropyridine (2a, 2.0 $\mathrm{mL}, 11 \mathrm{mmol}, 9.1$ equiv). The mixture was heated to $70^{\circ} \mathrm{C}$ and stirred for 2 days. The volatiles were then removed in vacuo. The resultant solid was dissolved in a minimal amount of hot diethyl ether. The solution was filtered through a syringe filter and cooled in a freezer to afford the product as green crystals $(0.373 \mathrm{~g}, 0.879$ mmol, 74.8\%). ${ }^{1} \mathrm{H}$ NMR (500 MHz, $\left.\mathrm{CDCl}_{3}\right): \delta 7.40(\mathrm{~d}, J=7.7 \mathrm{~Hz}, 2 \mathrm{H}), 7.05(\mathrm{t}, J=7.7 \mathrm{~Hz}, 2 \mathrm{H}), 7.00(\mathrm{t}, J=$ $7.7 \mathrm{~Hz}, 2 \mathrm{H}), 6.91$ (d, $J=7.7 \mathrm{~Hz}, 2 \mathrm{H}), 3.42$ (d, $J=10.4 \mathrm{~Hz}, 6 \mathrm{H}) \mathrm{ppm} .{ }^{13} \mathrm{C}\left\{{ }^{1} \mathrm{H}\right\} \mathrm{NMR}\left(126 \mathrm{MHz}, \mathrm{CDCl}_{3}\right): \delta$ 144.8-138.7 (m), 134.0 (dd, $J=23.0,2.8 \mathrm{~Hz}), 132.9$ (d, $J=12.9 \mathrm{~Hz}), 121.8(\mathrm{~s}), 120.8(\mathrm{~s}), 111.6(\mathrm{~d}, J=$ $8.7 \mathrm{~Hz}$ ), $110.2(\mathrm{dd}, J=12.0,2.8 \mathrm{~Hz}), 33.6(\mathrm{dm}, J=15.8 \mathrm{~Hz}) \mathrm{ppm} .{ }^{19} \mathrm{~F} \mathrm{NMR}\left(471 \mathrm{MHz}, \mathrm{CDCl}_{3}\right): \delta-34.67$ $\left(\mathrm{dt},{ }^{1} \mathrm{~J}_{\mathrm{F}-\mathrm{P}}=765.7 \mathrm{~Hz},{ }^{4} J_{\mathrm{F}-\mathrm{F}}=10.2 \mathrm{~Hz}\right),-90.51(\mathrm{~m}),-138.05(\mathrm{~m})$ ppm. ${ }^{31} \mathrm{P}$ NMR $\left(203 \mathrm{MHz}, \mathrm{CDCl}_{3}\right): \delta-61.04$ 
$\left(\mathrm{dm},{ }^{1} J_{\mathrm{P}-\mathrm{F}}=768.8 \mathrm{~Hz}\right)$ ppm. HRMS (DART) calculated for $\mathrm{C}_{19} \mathrm{H}_{15} \mathrm{~F}_{5} \mathrm{~N}_{4} \mathrm{P}[\mathrm{M}+\mathrm{H}]+425.09544$, found 425.09747.

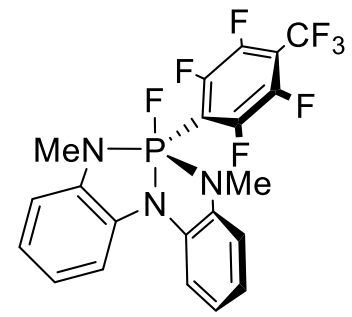

1• $[\mathrm{F}]\left[\mathrm{C}_{6} \mathrm{~F}_{4} \mathrm{CF}_{3}\right]$ : Inside a nitrogen filled glove box, to a vial containing a stir bar were added 1 ( $0.300 \mathrm{~g}, 1.18 \mathrm{mmol}, 1$ equiv) and octafluorotoluene ( $2 \mathbf{b}$, $2.0 \mathrm{~mL}, 14 \mathrm{mmol}, 12$ equiv). The mixture was heated to $90^{\circ} \mathrm{C}$ and stirred for 14 days to give precipitation. The remaining solution was decanted, and the precipitation was dried in vacuo. The resultant solid was dissolved in a minimal amount of hot diethyl ether. The solution was filtered through a syringe filter and cooled in a freezer to afford the product as green crystals $(0.368 \mathrm{~g}, 0.749 \mathrm{mmol}, 63.7 \%) .{ }^{1} \mathrm{H}$ NMR (500 MHz, $\left.\mathrm{CDCl}_{3}\right): \delta 7.39(\mathrm{~d}, J=7.7 \mathrm{~Hz}, 2 \mathrm{H}), 7.04(\mathrm{t}, J=7.7 \mathrm{~Hz}, 2 \mathrm{H}), 6.99(\mathrm{t}, J=7.7 \mathrm{~Hz}, 2 \mathrm{H}), 6.90(\mathrm{~d}, J=7.6 \mathrm{~Hz}, 2 \mathrm{H})$, $3.41(\mathrm{~d}, J=10.3 \mathrm{~Hz}, 6 \mathrm{H})$ ppm. ${ }^{13} \mathrm{C}\left\{{ }^{1} \mathrm{H}\right\}$ NMR $\left(126 \mathrm{MHz}, \mathrm{CDCl}_{3}\right): \delta 146.1-142.7(\mathrm{~m}), 134.2$ (dd, $J=23.0$, $2.9 \mathrm{~Hz}$ ), 133.0 (d, J = 12.9 Hz), 121.7 (s), 120.6 (s), 111.6 (d, J = 8.7 Hz), 110.1 (dd, $J=11.9,2.8 \mathrm{~Hz}$ ), $33.6(\mathrm{dm}, J=15.9 \mathrm{~Hz})$ ppm. ${ }^{19} \mathrm{~F}$ NMR $\left(471 \mathrm{MHz}, \mathrm{CDCl}_{3}\right): \delta-34.10\left(\mathrm{dt},{ }^{1} J_{\mathrm{F}-\mathrm{P}}=768.3 \mathrm{~Hz},{ }^{4} J_{\mathrm{F}-\mathrm{F}}=13.2 \mathrm{~Hz}\right)$, $-56.70(\mathrm{~m}),-134.45(\mathrm{~m}),-139.63(\mathrm{~m})$ ppm. ${ }^{31} \mathrm{P}$ NMR $\left(203 \mathrm{MHz}, \mathrm{CDCl}_{3}\right): \delta-60.10\left(\mathrm{dm},{ }^{1} \mathrm{P}_{\mathrm{P}-\mathrm{F}}=769.4 \mathrm{~Hz}\right)$ ppm. HRMS (DART) calculated for $\mathrm{C}_{21} \mathrm{H}_{15} \mathrm{~F}_{8} \mathrm{~N}_{3} \mathrm{P}[\mathrm{M}+\mathrm{H}]+492.08756$, found 492.09286 .

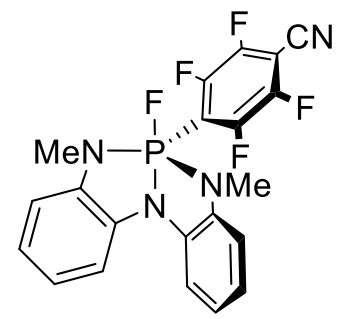

1• $[\mathrm{F}]\left[\mathrm{C}_{6} \mathrm{~F}_{4} \mathrm{CN}\right]$ : Inside a nitrogen filled glove box, to a vial containing a stir bar were added 1 ( $0.300 \mathrm{~g}, 1.18 \mathrm{mmol}, 1$ equiv) and pentafluorobenzonitrile (1.5 $\mathrm{mL}, 12 \mathrm{mmol}, 10$ equiv). The mixture was heated to $50{ }^{\circ} \mathrm{C}$ and stirred for 5 days. The volatiles were then removed in vacuo. The resultant solid was dissolved in a minimal amount of hot diethyl ether. The solution was filtered through a syringe filter and cooled in a freezer to afford the product as green crystals $(0.301 \mathrm{~g}, 0.671$ mmol, 57.1\%). ${ }^{1} \mathrm{H}$ NMR (500 MHz, $\left.\mathrm{CDCl}_{3}\right): \delta 7.38(\mathrm{~d}, J=7.7 \mathrm{~Hz}, 2 \mathrm{H}), 7.04(\mathrm{t}, J=7.6 \mathrm{~Hz}, 2 \mathrm{H}), 6.99(\mathrm{t}, J=$ $7.6 \mathrm{~Hz}, 2 \mathrm{H}), 6.89$ (d, $J=7.7 \mathrm{~Hz}, 2 \mathrm{H}), 3.40$ (d, $J=10.4 \mathrm{~Hz}, 6 \mathrm{H}) \mathrm{ppm} .{ }^{13} \mathrm{C}\left\{{ }^{1} \mathrm{H}\right\}$ NMR $\left(126 \mathrm{MHz}, \mathrm{CDCl}_{3}\right): \delta$ 148.1-143.6 (m), 134.0 (dd, $J=22.8,3.0 \mathrm{~Hz}), 132.9$ (d, $J=13.0 \mathrm{~Hz}), 121.8$ (s), 120.8 (s), 111.6 (d, $J=$ $8.7 \mathrm{~Hz}), 110.2(\mathrm{dd}, J=12.0,2.8 \mathrm{~Hz}), 107.4(\mathrm{t}, J=3.8 \mathrm{~Hz}), 33.6(\mathrm{dm}, J=15.7 \mathrm{~Hz}) \mathrm{ppm} .{ }^{19} \mathrm{~F}$ NMR $(471$ $\left.\mathrm{MHz}, \mathrm{CDCl}_{3}\right): \delta-33.91\left(\mathrm{dt},{ }^{1} J_{\mathrm{F}-\mathrm{P}}=769.6 \mathrm{~Hz},{ }^{4} J_{\mathrm{F}-\mathrm{F}}=12.0 \mathrm{~Hz}\right),-131.45(\mathrm{~m}),-133.45(\mathrm{~m}) \mathrm{ppm} .{ }^{31} \mathrm{P}$ NMR $\left(203 \mathrm{MHz}, \mathrm{CDCl}_{3}\right): \delta-60.20\left(\mathrm{dm},{ }^{1} J_{\mathrm{P}-\mathrm{F}}=769.1 \mathrm{~Hz}\right) \mathrm{ppm}$. HRMS (DART) calculated for $\mathrm{C}_{21} \mathrm{H}_{15} \mathrm{~F}_{5} \mathrm{~N}_{4} \mathrm{P}$ $[\mathrm{M}+\mathrm{H}]+449.09544$, found 449.09698 .

\footnotetext{
* Reactions can also be performed with lesser amount (>2 eq.) of perfluoroarene and longer reaction time in tetrahydrofuran.
} 


\section{c) Preparation of Organoaluminum Reagents}

A diisobutylaluminum hydride (DIBAL-H) solution (1.0 M in toluene) was purchased from Sigma Aldrich and used as received.

Diisobutylaluminum deuteride (DIBAL-D): Synthesized according to a modified literature procedure. ${ }^{8}$ Inside a nitrogen filled glove box, to a $25 \mathrm{~mL}$ Schlenk flask containing a stir bar were added lithium deuteride (0.179 g, $20.0 \mathrm{mmol}, 2.07$ equiv) and diethyl ether (10 mL). Diisobutylaluminum chloride (1.88 mL, $9.63 \mathrm{mmol}, 1$ equiv) was then added dropwise via a syringe with vigorous stirring. The flask was sealed, taken out of the glove box, connected to a Schlenk line, and quickly equipped with a reflux condenser with both the flask and the condenser under strong nitrogen stream. The reaction mixture was heated to reflux and stirred for 48 hours. The flask was brought into the glove box again, and the reaction mixture was filtered through celite. The filtrate was concentrated in vacuo to give a viscous liquid, which was then refiltered through celite. The product was used for a reaction with 1 $[\mathrm{F}]\left[\mathrm{C}_{5} \mathrm{~F}_{4} \mathrm{~N}\right]$ without further purification. ${ }^{1} \mathrm{H}$ NMR $\left(500 \mathrm{MHz}\right.$, toluene- $\left.\mathrm{d}_{8}\right): \delta 3.25(\mathrm{q}, J=7.1 \mathrm{~Hz}, 4 \mathrm{H}$, coordinating $\left.\mathrm{Et}_{2} \mathrm{O}\right), 2.21(\mathrm{~m}, 2 \mathrm{H}), 1.29(\mathrm{~d}, J=6.5 \mathrm{~Hz}, 12 \mathrm{H}), 1.02\left(\mathrm{t}, J=7.0 \mathrm{~Hz}, 6 \mathrm{H}\right.$, coordinating Et $\mathrm{O}_{2}$ ), $0.42(\mathrm{~d}, J=7.0 \mathrm{~Hz}, 4 \mathrm{H}) \mathrm{ppm}$.

Tris(pentafluorophenyl)aluminum: Synthesized according to modified literature procedures.9,10 Inside a nitrogen filled glove box, to a vial containing a stir bar were added tris(pentafluorophenyl)borane (0.388 g, $0.758 \mathrm{mmol}, 1$ equiv) and heptane (7.6 mL). A solution of triethylaluminum (1.0 M in hexane, $0.76 \mathrm{~mL}, 0.76 \mathrm{mmol}, 1.0$ equiv) was charged slowly. The resulting suspension was stirred at room temperature for 48 hours until it got thicker with white solids. The solid was filtered, washed with heptane, and dried in vacuo. The product was obtained as white powder (0.340 g, $0.644 \mathrm{mmol}, 84.9 \%$ ). ${ }^{19} \mathrm{~F}$ NMR (376 MHz, $\mathrm{C}_{6} \mathrm{D}_{6}$ ): $\delta-122.99$ (dd, $J=27.5,11.1 \mathrm{~Hz}$ ), $150.60(\mathrm{t}, J=20.2 \mathrm{~Hz}),-160.47(\mathrm{~m}) \mathrm{ppm}$.

\section{d) Synthesis of $1 \cdot[H]\left[C_{5} F_{4} E\right]$ via Ligand Metathesis}
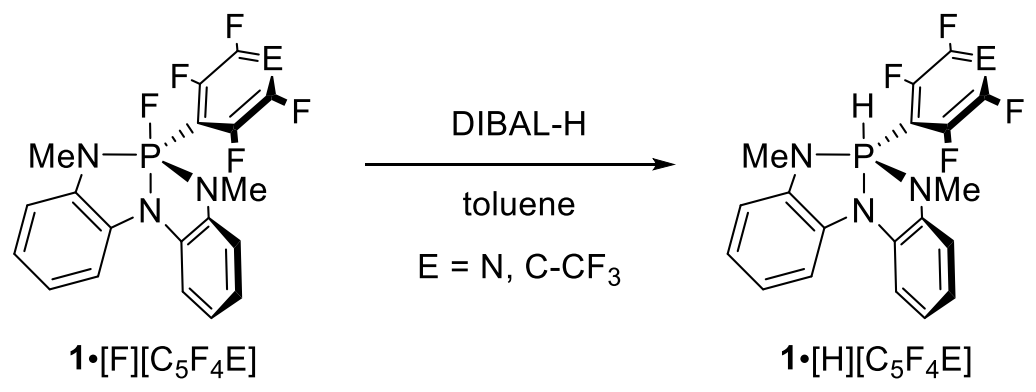
SAFETY NOTE: There have been reports of explosion from large-scale reactions ( $>1$ mol) between alane $\left(\mathrm{AlH}_{3}\right)$ and fluorinated compounds. ${ }^{11}$ Although DIBAL-H is less reactive than alane, all possible precautions should be taken when performing the following reactions.

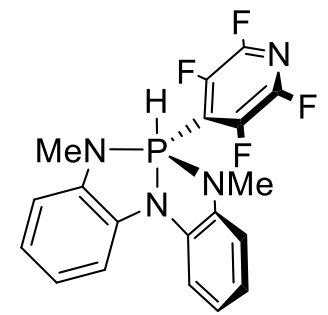

1• $[\mathrm{H}]\left[\mathrm{C}_{5} \mathrm{~F}_{4} \mathrm{~N}\right]$ : Inside a nitrogen filled glove box, to a vial containing a stir bar were added $\mathbf{1} \cdot[\mathrm{F}]\left[\mathrm{C}_{5} \mathrm{~F}_{4} \mathrm{~N}\right](0.435 \mathrm{~g}, 1.03 \mathrm{mmol}, 1$ equiv) and toluene $(2.0 \mathrm{~mL})$. To this solution was added a solution of DIBAL-H (1.0 M in toluene, $1.5 \mathrm{~mL}, 1.5$ mmol, 1.5 equiv). The reaction mixture was stirred at room temperature for 1 hour. The solvent was then removed in vacuo, and the resultant solid was washed with pentane and dried again. A minimal amount of hot diethyl ether was added to completely dissolve the solid. The solution was filtered through a syringe filter into another vial and cooled in a freezer to afford the product as pale-yellow crystals $(0.238 \mathrm{~g}, 0.586 \mathrm{mmol}, 57.1 \%) .{ }^{1} \mathrm{H}$ $\operatorname{NMR}\left(500 \mathrm{MHz}, \mathrm{CDCl}_{3}\right): \delta 7.46(\mathrm{~d}, J=7.8 \mathrm{~Hz}, 2 \mathrm{H}), 7.16\left(\mathrm{~d},{ }^{1} \mathrm{~J}_{\mathrm{H}-\mathrm{P}}=542.4 \mathrm{~Hz}, 1 \mathrm{H}\right), 7.03-6.96(\mathrm{~m}, 4 \mathrm{H}), 6.82$ $(\mathrm{d}, J=7.2 \mathrm{~Hz}, 2 \mathrm{H}), 3.17(\mathrm{~d}, J=17.6 \mathrm{~Hz}, 6 \mathrm{H}) \mathrm{ppm} .{ }^{13} \mathrm{C}\left\{{ }^{1} \mathrm{H}\right\}$ NMR $\left(126 \mathrm{MHz}, \mathrm{CDCl}_{3}\right): \delta 144.8-139.9(\mathrm{~m})$, $132.2(\mathrm{~d}, J=13.7 \mathrm{~Hz}), 131.7$ (d, $J=15.9 \mathrm{~Hz}), 121.1$ (s), 120.5 (d, $J=1.7 \mathrm{~Hz}), 111.0$ (d, J = 10.4 Hz), 109.6 (d, $J=7.8 \mathrm{~Hz}$ ), $29.2\left(\mathrm{dm}, J=16.8 \mathrm{~Hz}\right.$ ) ppm. ${ }^{19} \mathrm{~F} \mathrm{NMR} \mathrm{(471} \mathrm{MHz,} \mathrm{CDCl}_{3}$ ): $\delta-91.00(\mathrm{~m}),-137.97$ (m) ppm. ${ }^{31} \mathrm{P}$ NMR $\left(203 \mathrm{MHz}, \mathrm{CDCl}_{3}\right): \delta-62.67\left(\mathrm{dm},{ }^{1} \mathrm{~J}_{\mathrm{P}-\mathrm{H}}=541.7 \mathrm{~Hz}\right) \mathrm{ppm} .{ }^{31} \mathrm{P}\left\{{ }^{1} \mathrm{H}\right\} \mathrm{NMR}\left(203 \mathrm{MHz}, \mathrm{CDCl}_{3}\right): \delta-$ 62.67 (m) ppm. HRMS (DART) calculated for $\mathrm{C}_{19} \mathrm{H}_{16} \mathrm{~F}_{4} \mathrm{~N}_{4} \mathrm{P}[\mathrm{M}+\mathrm{H}]+407.10486$, found 407.10617.

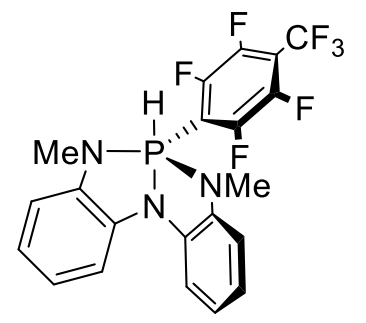

1•[H] $\left[\mathrm{C}_{6} \mathrm{~F}_{4} \mathrm{CF}_{3}\right]$ : Inside a nitrogen filled glove box, to a vial containing a stir bar were added $1 \cdot[\mathrm{F}]\left[\mathrm{C}_{6} \mathrm{~F}_{4} \mathrm{CF}_{3}\right]$ (0.202 g, $0.411 \mathrm{mmol}, 1$ equiv) and toluene (1.0 mL). To this solution was added a solution of DIBAL-H (1.0 M in toluene, $0.62 \mathrm{~mL}, 0.62 \mathrm{mmol}, 1.5$ equiv). The reaction mixture was stirred at room temperature for 1 hour. The solvent was then removed in vacuo, and the resultant solid was washed with pentane and dried again. A minimal amount of hot diethyl ether was added to completely dissolve the solid. The solution was filtered through a syringe filter into another vial and cooled in a freezer to afford the product as pale-green crystals (0.103 g, $0.218 \mathrm{mmol}, 52.9 \%)$. ${ }^{1} \mathrm{H} \mathrm{NMR}\left(500 \mathrm{MHz}, \mathrm{CDCl}_{3}\right): \delta 7.46(\mathrm{~d}, J=7.4 \mathrm{~Hz}, 2 \mathrm{H}), 7.16\left(\mathrm{~d},{ }^{1} \mathrm{~J}_{\mathrm{H}-\mathrm{P}}=541.9 \mathrm{~Hz}, 1 \mathrm{H}\right), 7.03-6.96(\mathrm{~m}, 4 \mathrm{H})$, $6.82(\mathrm{~d}, J=7.4 \mathrm{~Hz}, 2 \mathrm{H}), 3.17(\mathrm{~d}, J=17.7 \mathrm{~Hz}, 6 \mathrm{H}) \mathrm{ppm} .{ }^{13} \mathrm{C}\left\{{ }^{1} \mathrm{H}\right\}$ NMR $\left(126 \mathrm{MHz}, \mathrm{CDCl}_{3}\right): \delta$ 147.2-142.7 (m), 132.4 (d, J = $13.8 \mathrm{~Hz}), 131.8$ (d, $J=15.8 \mathrm{~Hz}), 121.0$ (s), 120.3 (s), 110.9 (d, $J=10.3 \mathrm{~Hz}$ ), 109.5 (d, $J=7.6 \mathrm{~Hz}), 29.3(\mathrm{dm}, J=16.6 \mathrm{~Hz}) \mathrm{ppm} .{ }^{19} \mathrm{~F} \mathrm{NMR}\left(471 \mathrm{MHz}, \mathrm{CDCl}_{3}\right): \delta-56.76(\mathrm{~m}),-134.81(\mathrm{~m}),-139.90$ (m) ppm. ${ }^{31} \mathrm{P}$ NMR $\left(203 \mathrm{MHz}, \mathrm{CDCl}_{3}\right): \delta-62.22\left(\mathrm{dm},{ }^{1} \mathrm{P}_{\mathrm{P}-\mathrm{H}}=541.6 \mathrm{~Hz}\right) \mathrm{ppm} .{ }^{31} \mathrm{P}\left\{{ }^{1} \mathrm{H}\right\} \mathrm{NMR}(203 \mathrm{MHz}$, $\mathrm{CDCl}_{3}$ ): $\delta$-62.22 (m) ppm. HRMS (DART) calculated for $\mathrm{C}_{21} \mathrm{H}_{16} \mathrm{~F}_{7} \mathrm{~N}_{3} \mathrm{P}[\mathrm{M}+\mathrm{H}]^{+}$474.09701, found 474.10516. 


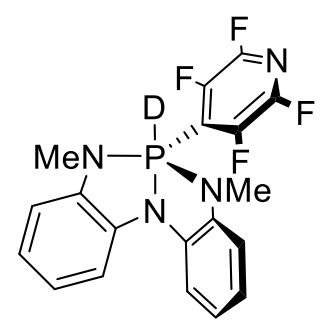

$\mathbf{1} \cdot[\mathrm{D}]\left[\mathrm{C}_{5} \mathrm{~F}_{4} \mathrm{~N}\right]$ : Inside a nitrogen filled glove box, to a vial was added $\mathbf{1} \cdot[\mathrm{F}]\left[\mathrm{C}_{5} \mathrm{~F}_{4} \mathrm{~N}\right]$ ( $0.068 \mathrm{~g}, 0.16 \mathrm{mmol}, 1$ equiv). A solution of DIBAL-D in toluene- $\mathrm{d}_{8}$ was added sequentially until full conversion was achieved. The solvent was then removed in vacuo, and the resultant solid was washed with pentane and dried again. A minimal amount of hot diethyl ether was added to completely dissolve the solid.

The solution was filtered through a syringe filter into another vial and cooled in a freezer to afford the product as pale-yellow crystals $(0.035 \mathrm{~g}, 0.086 \mathrm{mmol}, 54 \%) .{ }^{1} \mathrm{H}$ NMR (500 MHz, $\left.\mathrm{CDCl}_{3}\right): \delta 7.46(\mathrm{~d}$, $J=7.7 \mathrm{~Hz}, 2 \mathrm{H}), 7.03-6.96(\mathrm{~m}, 4 \mathrm{H}), 6.82(\mathrm{~d}, J=7.4 \mathrm{~Hz}, 2 \mathrm{H}), 3.17(\mathrm{~d}, J=17.6 \mathrm{~Hz}, 6 \mathrm{H}) \mathrm{ppm} .{ }^{13} \mathrm{C}\left\{{ }^{1} \mathrm{H}\right\} \mathrm{NMR}$ (126 MHz, $\mathrm{CDCl}_{3}$ ): $\delta$ 144.7-139.9 (m), 132.2 (d, J = 13.9 Hz), 131.7 (d, J = 15.8 Hz), 121.1 (s), 120.5 (d, $J=1.7 \mathrm{~Hz}), 111.0(\mathrm{~d}, J=10.5 \mathrm{~Hz}), 109.6(\mathrm{~d}, J=7.8 \mathrm{~Hz}), 29.2(\mathrm{dm}, J=16.5 \mathrm{~Hz}) \mathrm{ppm} .{ }^{19} \mathrm{~F} \mathrm{NMR}(471 \mathrm{MHz}$, $\left.\mathrm{CDCl}_{3}\right): \delta-91.02(\mathrm{~m}),-137.97$ ppm. ${ }^{31} \mathrm{P}$ NMR $\left(203 \mathrm{MHz}, \mathrm{CDCl}_{3}\right): \delta-63.22\left(\mathrm{tm},{ }^{1} \mathrm{~J}_{\mathrm{P}-\mathrm{D}}=82.1 \mathrm{~Hz}\right) \mathrm{ppm}$. HRMS (DART) calculated for $\mathrm{C}_{19} \mathrm{H}_{15} \mathrm{DF}_{4} \mathrm{~N}_{4} \mathrm{P}[\mathrm{M}+\mathrm{H}]+408.11114$, found 408.11490 .

* Attempts for the ligand metathesis from $\mathbf{1} \cdot[\mathrm{F}]\left[\mathrm{C}_{6} \mathrm{~F}_{4} \mathrm{CN}\right]$ resulted in multiple products as the nitrile group was also reactive to DIBAL-H. 


\section{C-H Reductive Elimination from $1 \cdot[H]\left[C_{5} F_{4} E\right]$}

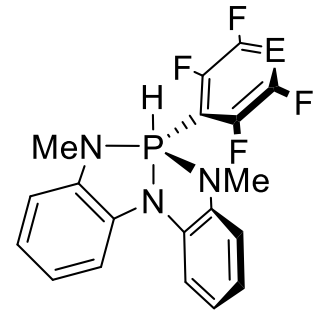

$1 \cdot[\mathrm{H}]\left[\mathrm{C}_{5} \mathrm{~F}_{4} \mathrm{E}\right]$

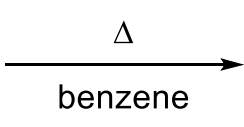

a) General Procedure for $\mathrm{C}-\mathrm{H}$ Reductive Elimination

A sealed J-Young NMR tube containing a solution of $\mathbf{1} \cdot[\mathrm{H} / \mathrm{D}]\left[\mathrm{C}_{5} \mathrm{~F}_{4} \mathrm{E}\right]\left(\mathrm{E}=\mathrm{N}, \mathrm{C}-\mathrm{CF}_{3}\right)$ in $\mathrm{C}_{6} \mathrm{D}_{6}(5-100$ $\mathrm{mM}$ ) was heated in an oil bath whose temperature was set to $140-180^{\circ} \mathrm{C}$. Products were analyzed by ${ }^{31} \mathrm{P}$ NMR (1) and ${ }^{19} \mathrm{~F}$ NMR (3a, 3b) spectra.

3a: ${ }^{1} \mathrm{H}$ NMR (500 MHz, $\mathrm{C}_{6} \mathrm{D}_{6}$ ): $\delta 5.98(\mathrm{~m}) \mathrm{ppm} .{ }^{19} \mathrm{~F}$ NMR (376 MHz, $\mathrm{C}_{6} \mathrm{D}_{6}$ ): $\delta-91.42(\mathrm{~m}, 2,6-\mathrm{F}),-139.96$ $(\mathrm{m}, 3,5-\mathrm{F}) \mathrm{ppm}$.

3a- $d:{ }^{19}$ F NMR (376 MHz, $\left.\mathrm{C}_{6} \mathrm{D}_{6}\right): \delta-91.25(\mathrm{~m}, 2,6-\mathrm{F}),-140.23(\mathrm{~m}, 3,5-\mathrm{F}) \mathrm{ppm}$.

3b: ${ }^{1} \mathrm{H}$ NMR (500 MHz, $\left.\mathrm{C}_{6} \mathrm{D}_{6}\right): \delta 5.92(\mathrm{~m})$ ppm. ${ }^{19} \mathrm{~F}$ NMR $\left(376 \mathrm{MHz}, \mathrm{C}_{6} \mathrm{D}_{6}\right): \delta-57.20(\mathrm{t}, J=21.8 \mathrm{~Hz}, \alpha-\mathrm{F})$, $-137.62(\mathrm{~m}, 3,5-\mathrm{F}),-142.19(\mathrm{~m}, 2,6-\mathrm{F}) \mathrm{ppm}$. 


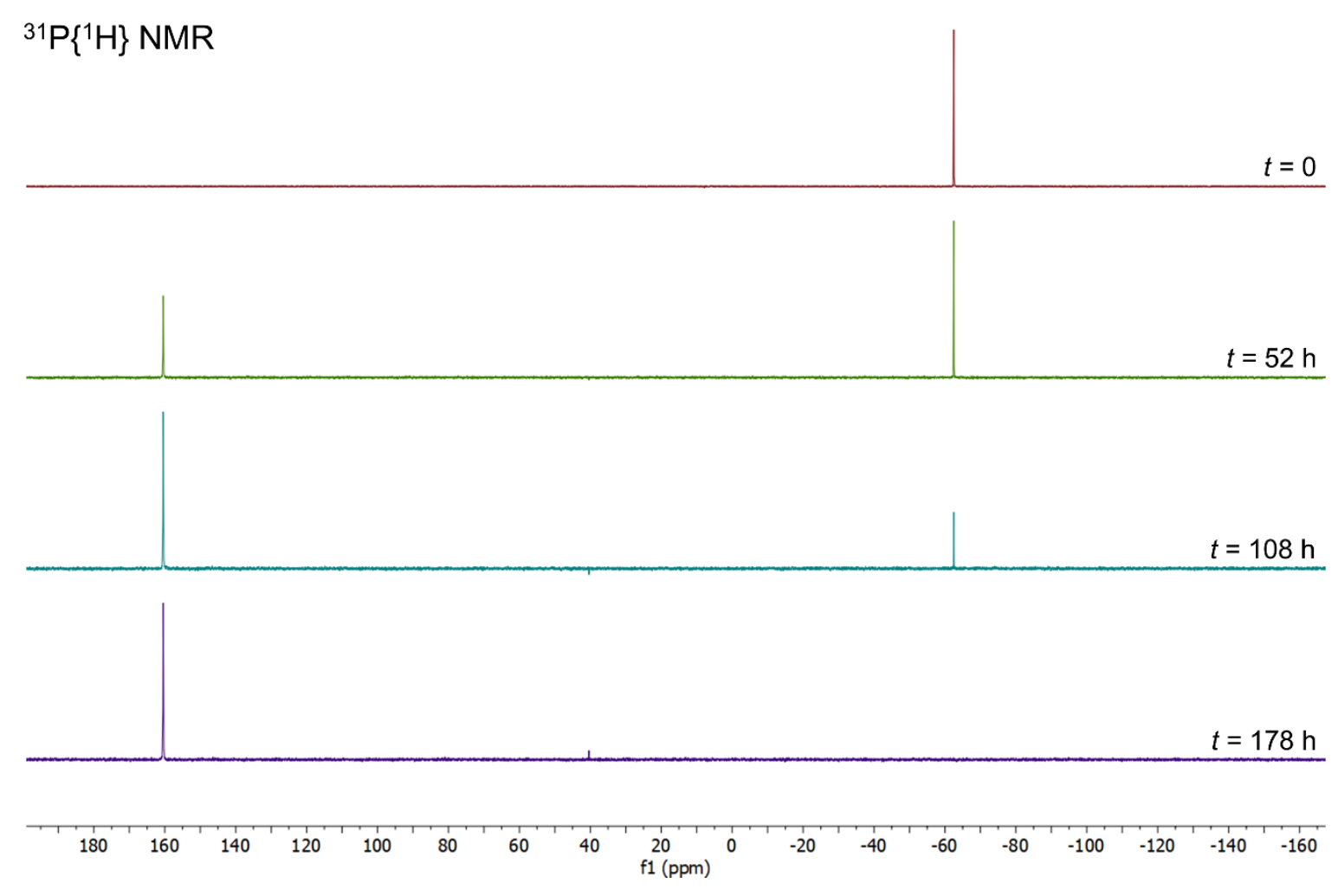

${ }^{19} \mathrm{~F}$ NMR $\mid$

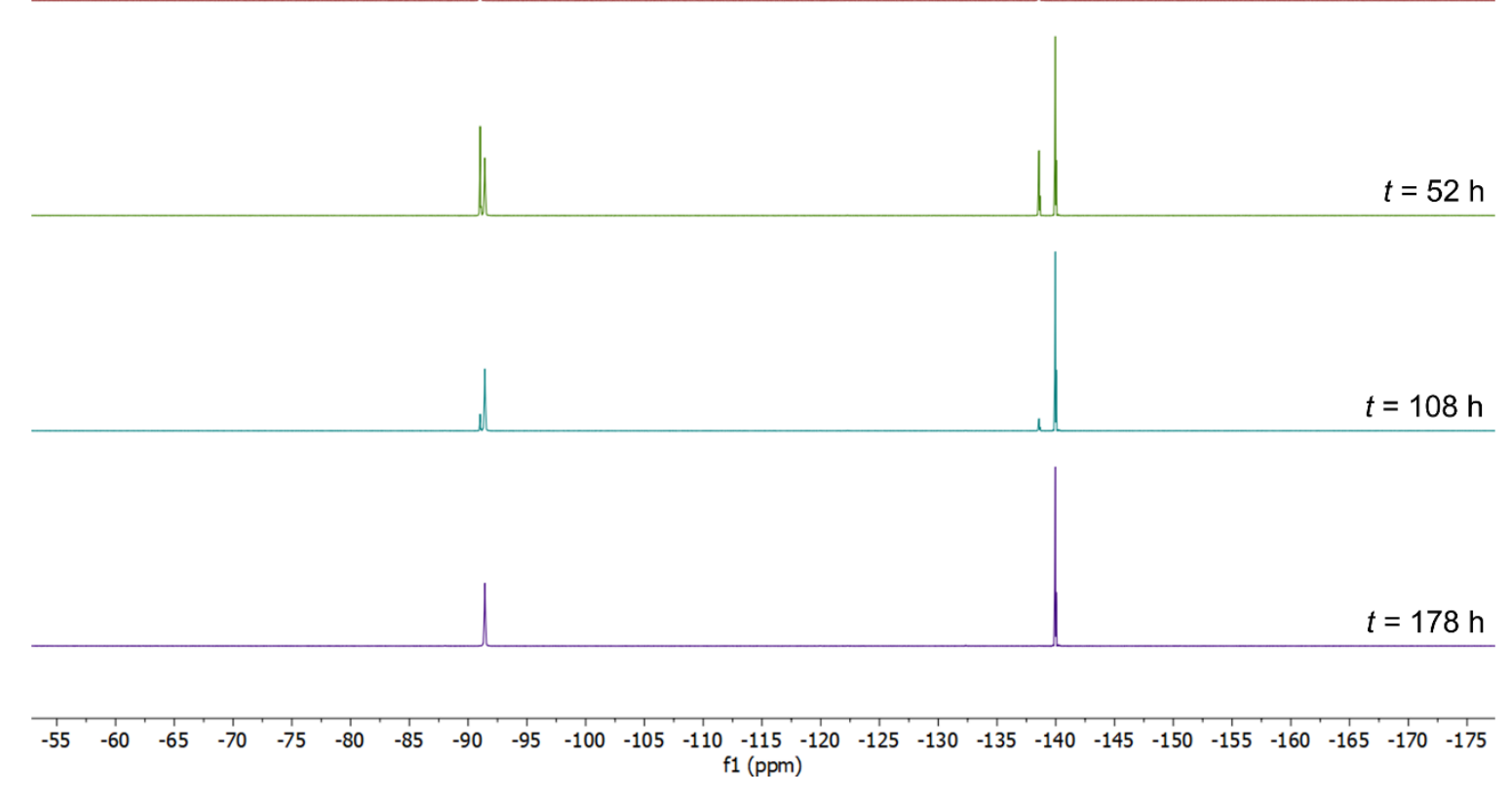

Figure S1. Time-stacked (top) ${ }^{31} \mathrm{P}\left\{{ }^{1} \mathrm{H}\right\}$ NMR $(203 \mathrm{MHz})$ and (bottom) ${ }^{19} \mathrm{~F}$ NMR spectra (471 MHz) of a solution of $1 \cdot[\mathrm{H}]\left[\mathrm{C}_{5} \mathrm{~F}_{4} \mathrm{~N}\right]$ in $\mathrm{C}_{6} \mathrm{D}_{6}$ heated in a $160^{\circ} \mathrm{C}$ oil bath for $t=0,52,108$, and $178 \mathrm{~h}$. Chemical shifts are relative to $85 \% \mathrm{H}_{3} \mathrm{PO}_{4}\left({ }^{31} \mathrm{P}\right)$ and $\mathrm{CFCl}_{3}\left({ }^{19} \mathrm{~F}\right)$. 

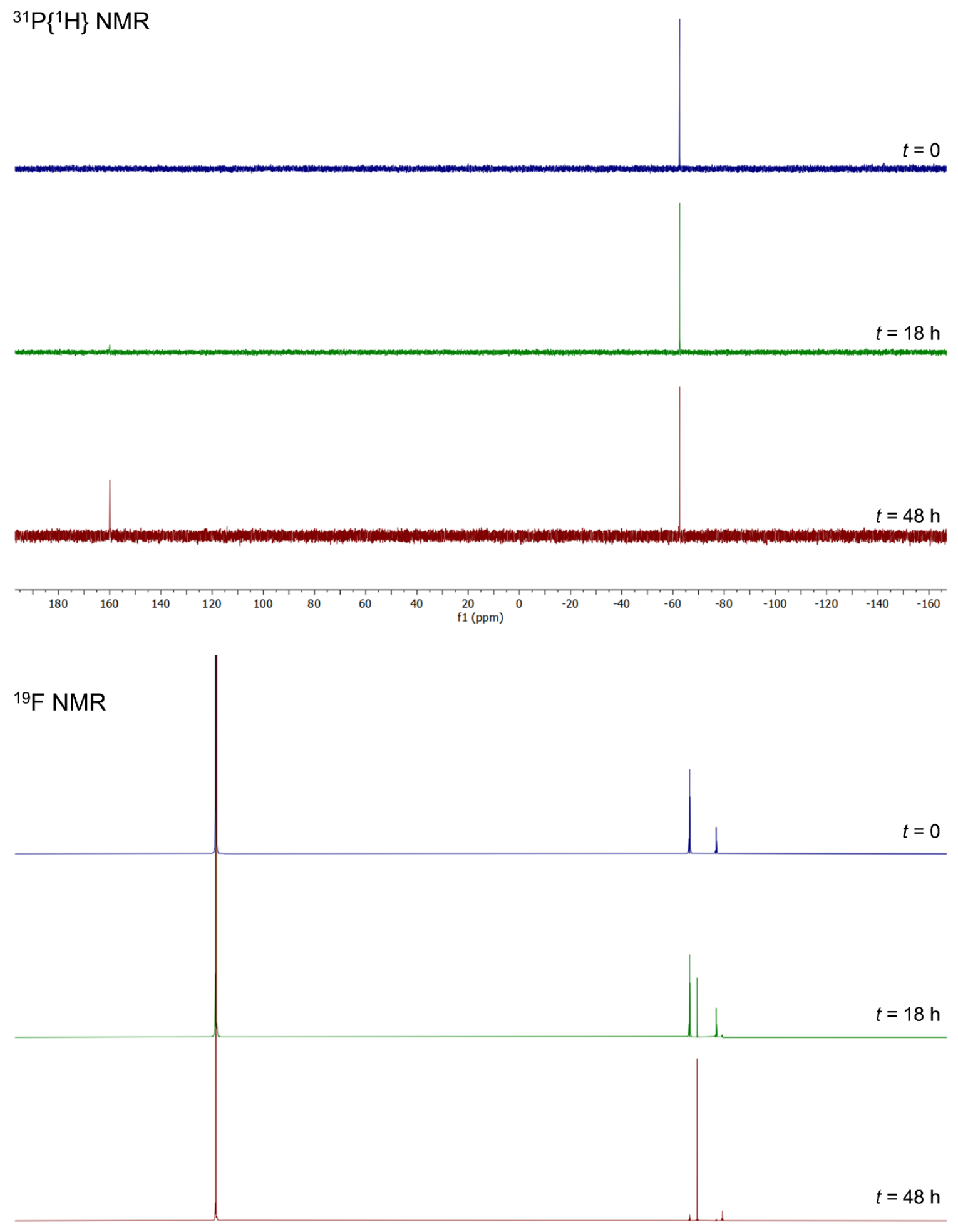

\begin{tabular}{rlllllllllllllllllllllllllllllllll}
\hline-25 & -30 & -35 & -40 & -45 & -50 & -55 & -60 & -65 & -70 & -75 & -80 & -85 & -90 & -95 & -100 & -105 & -110 & -115 & -120 & -125 & -130 & -135 & -140 & -145 & -150 & -155 & -160 & -165 & -170 & -175
\end{tabular}

Figure S2. Time-stacked (top) ${ }^{31} \mathrm{P}\left\{{ }^{1} \mathrm{H}\right\}$ NMR $(203 \mathrm{MHz})$ and (bottom) ${ }^{19} \mathrm{~F}$ NMR (471 MHz) spectra of a solution of $1 \cdot[\mathrm{H}]\left[\mathrm{C}_{6} \mathrm{~F}_{4} \mathrm{CF}_{3}\right]$ in $\mathrm{C}_{6} \mathrm{D}_{6}$ heated in a $160^{\circ} \mathrm{C}$ oil bath for $t=0,18$, and $48 \mathrm{~h}$. Chemical shifts are relative to $85 \% \mathrm{H}_{3} \mathrm{PO}_{4}\left({ }^{31} \mathrm{P}\right)$ and $\mathrm{CFCl}_{3}\left({ }^{19} \mathrm{~F}\right)$. 


\section{b) Hydrodefluorination Product Assignment}

The assignment of the hydrodefluorination products $\mathbf{3 a}$ and $\mathbf{3 b}$ was done by comparison with the NMR spectrum of a commercial product purchased from Sigma Aldrich (3a, Figure S3) or spin simulation using MestReNova (3a- $d$ and 3b, Figure S4, S5).

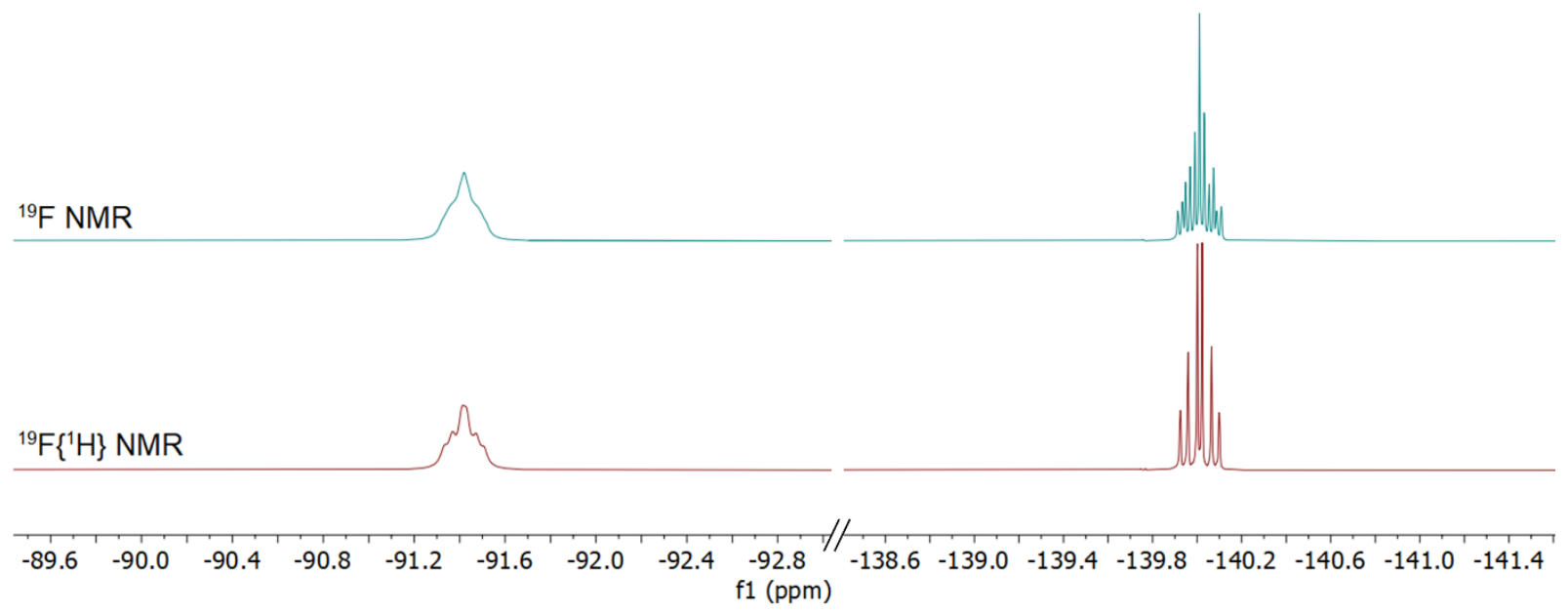

Figure S3. ${ }^{19} \mathrm{~F}$ and ${ }^{19} \mathrm{~F}\left\{{ }^{1} \mathrm{H}\right\}$ NMR (376 MHz) spectra of $3 \mathbf{a}$ in $\mathrm{C}_{6} \mathrm{D}_{6}$. Chemical shifts are relative to $\mathrm{CFCl}_{3}$.

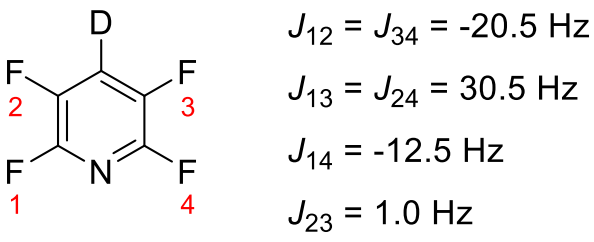

$3 a-d$

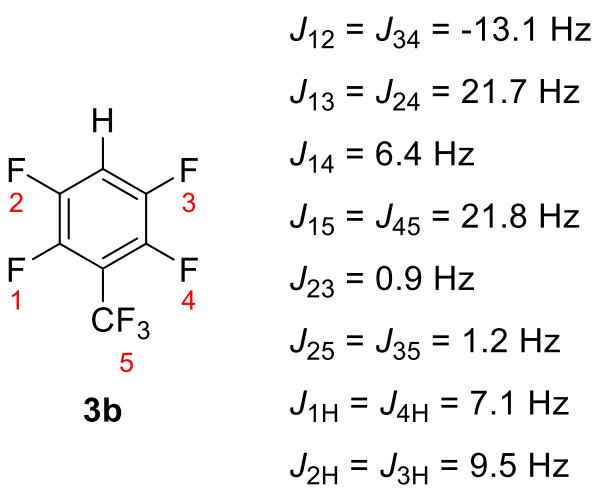

$J_{13}=J_{24}=21.7 \mathrm{~Hz}$

$J_{14}=6.4 \mathrm{~Hz}$

$J_{15}=J_{45}=21.8 \mathrm{~Hz}$

$23=0.9 \mathrm{~Hz}$

$J_{25}=J_{35}=1.2 \mathrm{~Hz}$

$J_{2 \mathrm{H}}=J_{3 \mathrm{H}}=9.5 \mathrm{~Hz}$

Figure S4. Simulated NMR coupling constants in $\mathbf{3 a -} d$ and $\mathbf{3 b}$. ${ }^{12}$ 
(a) ${ }^{19}$ F NMR

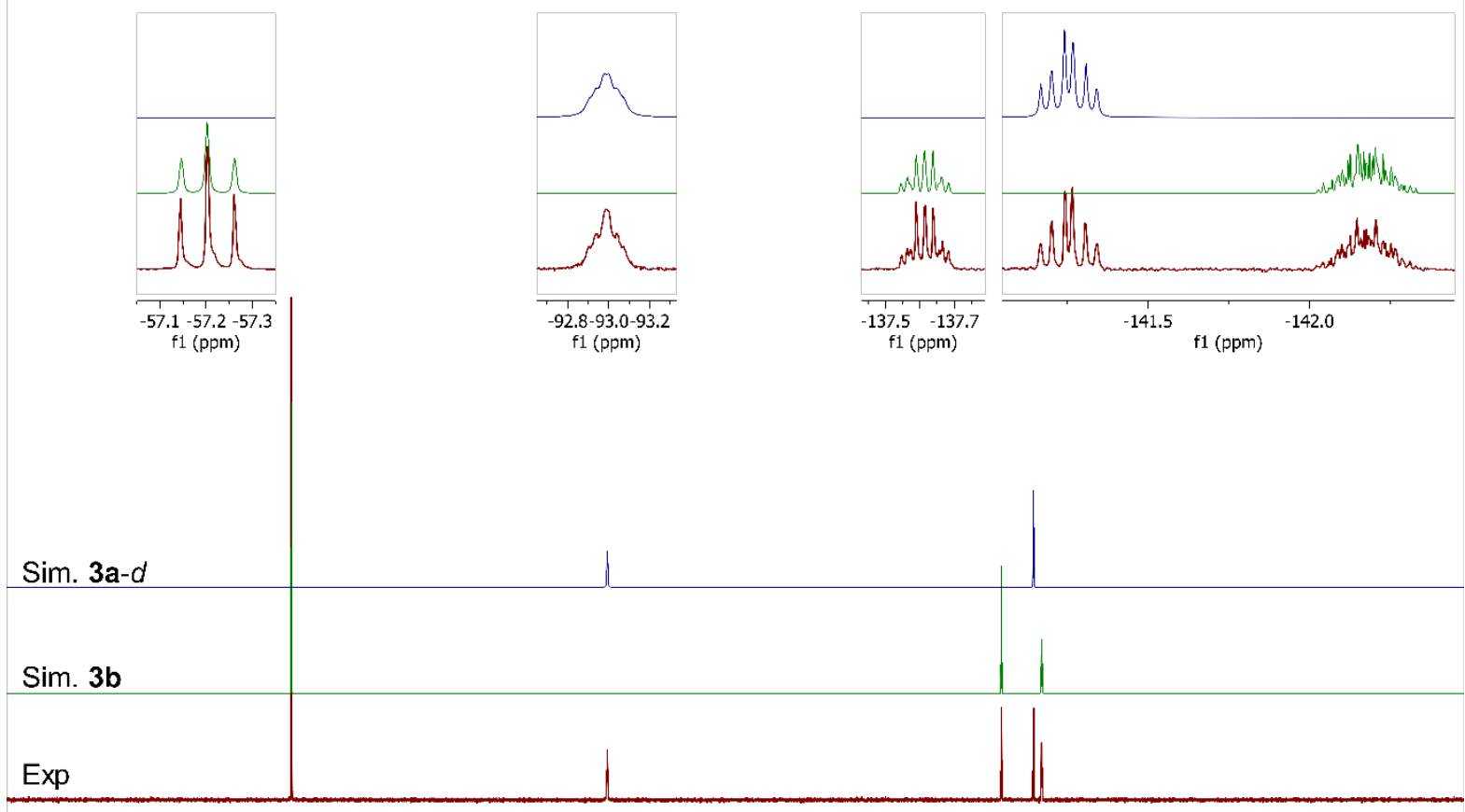

\begin{tabular}{lll}
\hline-50 & -100 & -150 \\
$f 1(\mathrm{ppm})$ & -150
\end{tabular}

(b) ${ }^{19} \mathrm{~F}\left\{{ }^{1} \mathrm{H}\right\} \mathrm{NMR}$

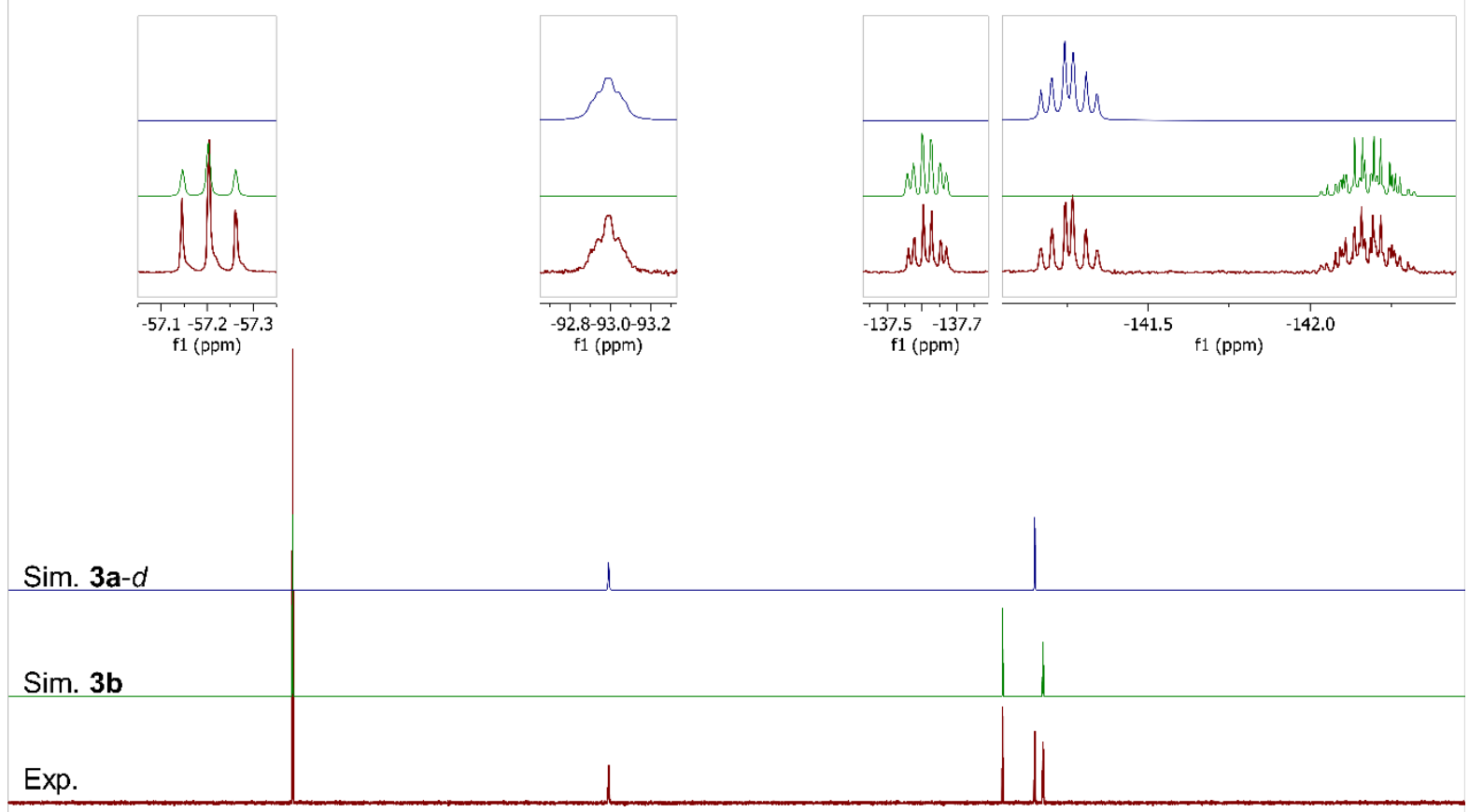

$-50$

$-100 \mathrm{f} 1(\mathrm{ppm})$

Figure S5. (a) ${ }^{19} \mathrm{~F}$ and (b) ${ }^{19} \mathrm{~F}\left\{{ }^{1} \mathrm{H}\right\}(376 \mathrm{MHz})$ NMR spectra after heating a solution of $\mathbf{3 a}-d$ and $\mathbf{3 b}$ in $\mathrm{C}_{6} \mathrm{D}_{6}$ in a $170{ }^{\circ} \mathrm{C}$ oil bath, stacked with simulated spectra of $\mathbf{3 a}-d$ and $\mathbf{3 b}$. Chemical shifts are relative to $\mathrm{CFCl}_{3}$. 


\section{c) Reaction Kinetics}

Reaction kinetics was measured at $160{ }^{\circ} \mathrm{C}$ in a sealed J-Young NMR tube enclosed in a stainless-steel tube that was connected to a remote-reading thermocouple probe and immersed in an insulated oil bath to ensure homogeneous temperature control. Concentrations of reactants and products were monitored by quantitative ${ }^{1} \mathrm{H}$ NMR spectroscopy using durene as an internal standard. Rate constants were extracted from the plot of $\ln \left(\left[\mathbf{1} \cdot[\mathrm{H} / \mathrm{D}]\left[\mathrm{C}_{5} \mathrm{~F}_{4} \mathrm{~N}\right]\right]\right)$ vs. time.

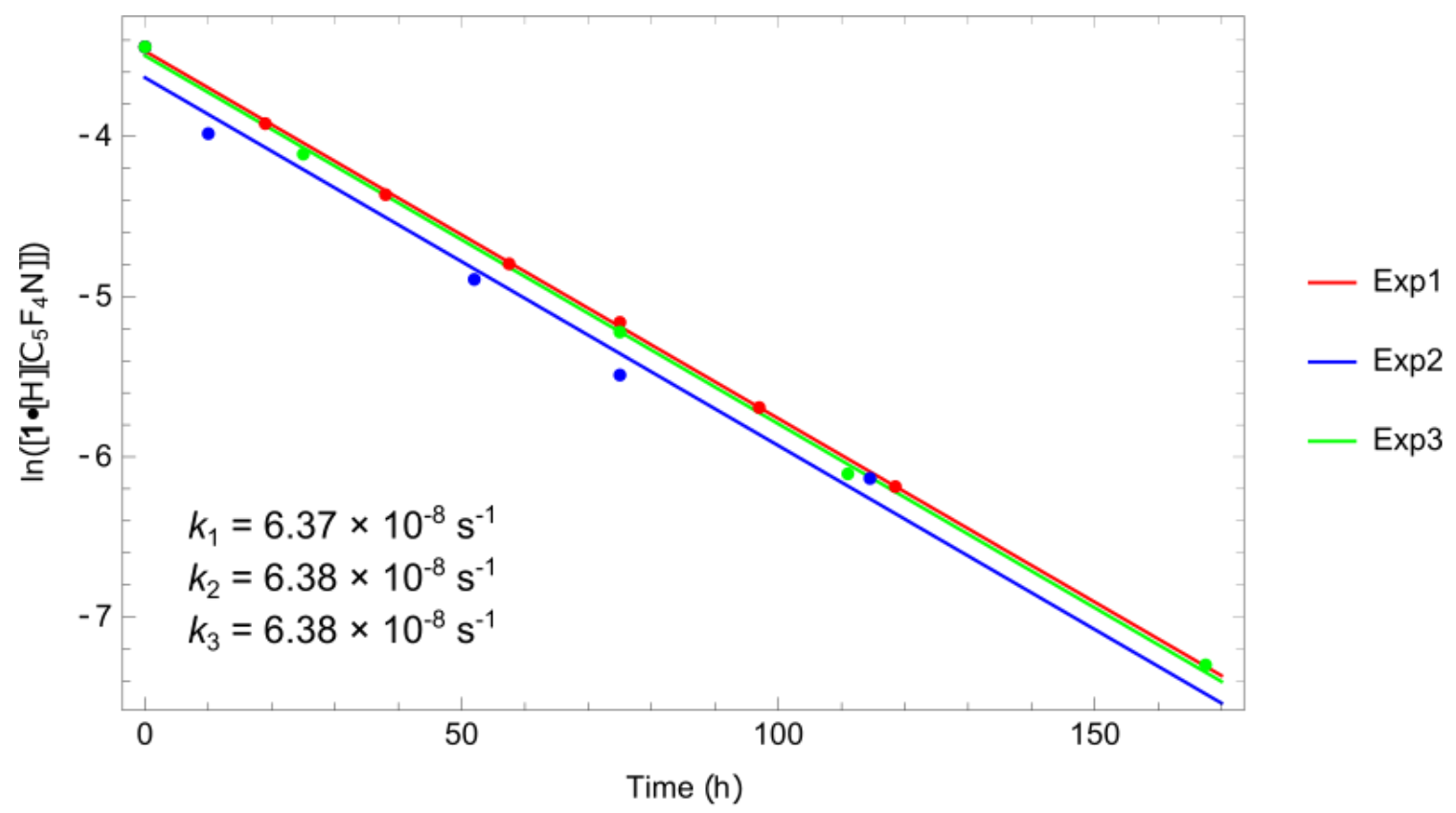

Figure S6. First-order decay of $1 \cdot[\mathrm{H}]\left[\mathrm{C}_{5} \mathrm{~F}_{4} \mathrm{~N}\right]$ at $160^{\circ} \mathrm{C}$ and its rate constants.

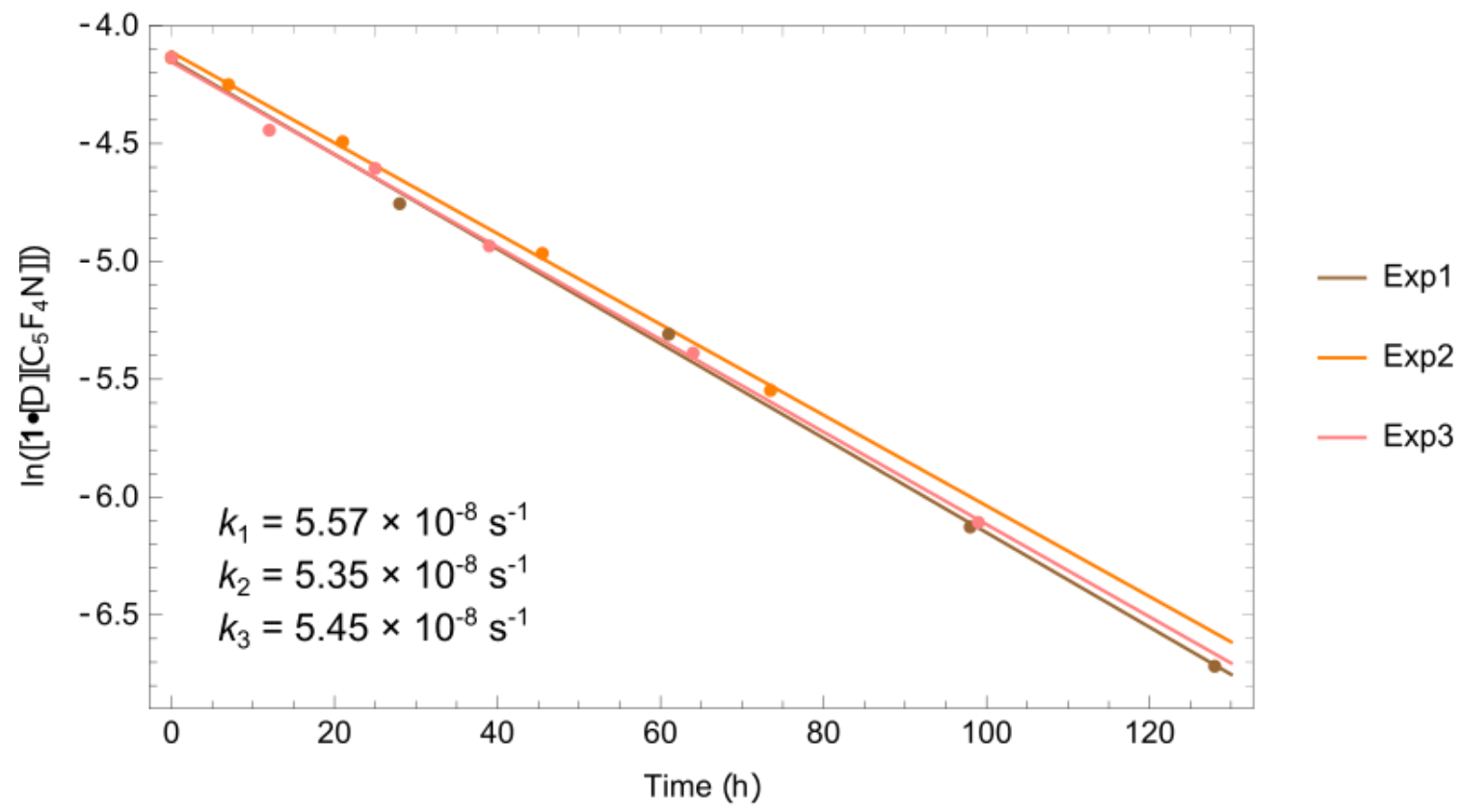

Figure S7. First-order decay of $\mathbf{1} \cdot[\mathrm{D}]\left[\mathrm{C}_{5} \mathrm{~F}_{4} \mathrm{~N}\right]$ at $160^{\circ} \mathrm{C}$ and its rate constants. 


\section{d) One-Pot Ligand Metathesis and Reductive Elimination}

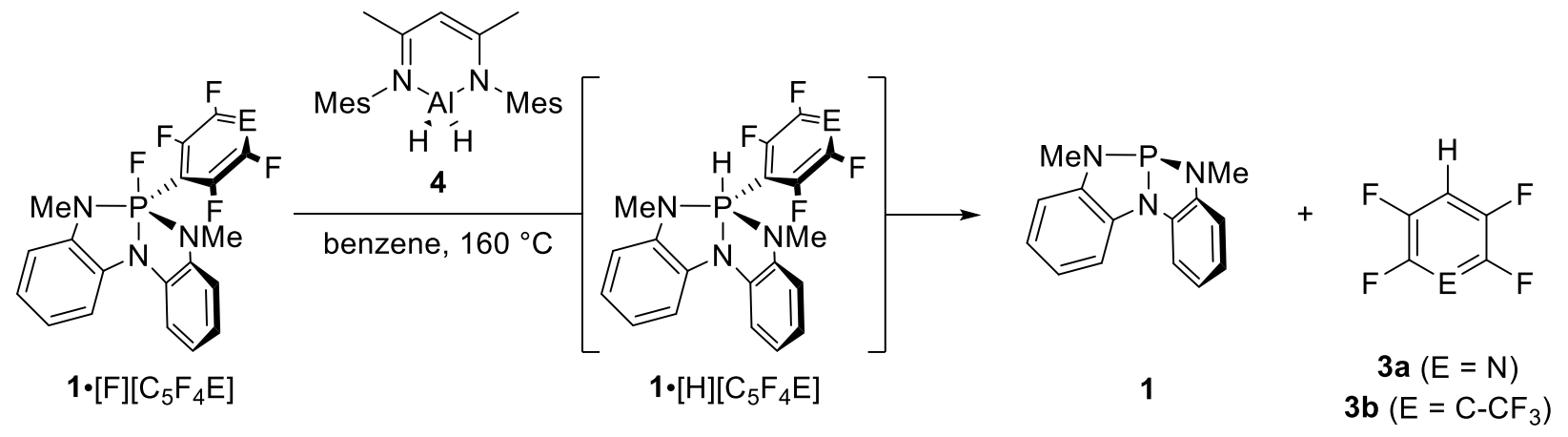

Diketiminate-stabilized aluminum dihydride 4 that is thermally stable at $160{ }^{\circ} \mathrm{C}$ was prepared according to a literature procedure. ${ }^{13}$ In $_{6} \mathrm{D}_{6}$, compound 4 promoted exchange of fluoride for hydride with heating above $120^{\circ} \mathrm{C}$. To demonstrate one-pot ligand metathesis and reductive elimination, a solution of $1 \cdot[\mathrm{F}]\left[\mathrm{C}_{5} \mathrm{~F}_{4} \mathrm{E}\right]\left(\mathrm{E}=\mathrm{N}, \mathrm{C}-\mathrm{CF}_{3}\right)$ and 4 (1.5 equiv) in $\mathrm{C}_{6} \mathrm{D}_{6}(0.020 \mathrm{M})$ was heated to $160{ }^{\circ} \mathrm{C}$ in a sealed J-Young NMR tube. As shown in Figure S8, the formation of both $\mathbf{1} \cdot[\mathrm{H}]\left[\mathrm{C}_{5} \mathrm{~F}_{4} \mathrm{E}\right]$ and $\mathrm{H}-\mathrm{C}_{5} \mathrm{~F}_{4} \mathrm{E}$ was observed by NMR. Considering that $\mathbf{1 \bullet}[\mathrm{F}]\left[\mathrm{C}_{5} \mathrm{~F}_{4} \mathrm{E}\right]$ alone does not undergo the extrusion of $\mathrm{F}-$ $\mathrm{C}_{5} \mathrm{~F}_{4} \mathrm{E}$ at $160{ }^{\circ} \mathrm{C}$, the observed result indicates the compatibility of ligand metathesis and reductive elimination using this thermally stable aluminum hydride.

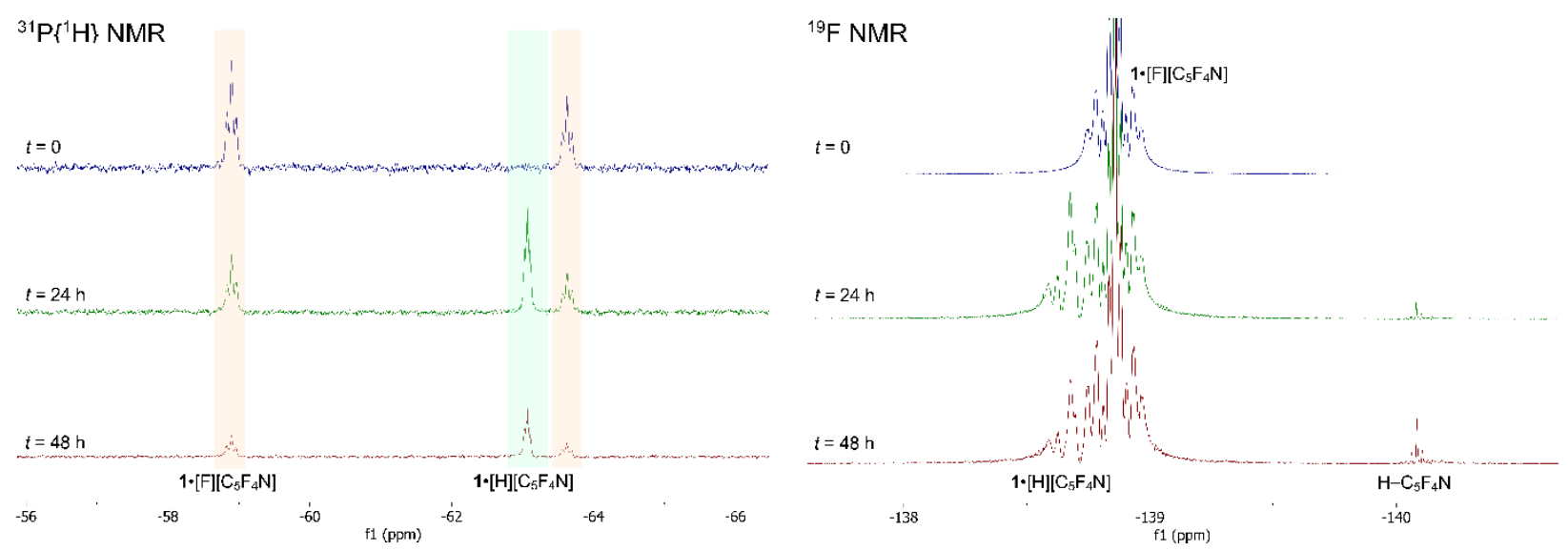

Figure S8. Time-stacked (left) ${ }^{31} \mathrm{P}\left\{{ }^{1} \mathrm{H}\right\}$ NMR $(203 \mathrm{MHz})$ and (right) ${ }^{19} \mathrm{~F}$ NMR (471 MHz) spectra of a solution of $1 \cdot[\mathrm{F}]\left[\mathrm{C}_{5} \mathrm{~F}_{4} \mathrm{~N}\right]$ and 4 in $\mathrm{C}_{6} \mathrm{D}_{6}$ heated in a $160^{\circ} \mathrm{C}$ oil bath for $t=0,24$, and $48 \mathrm{~h}$. Chemical shifts are relative to $85 \% \mathrm{H}_{3} \mathrm{PO}_{4}\left({ }^{31} \mathrm{P}\right)$ and $\mathrm{CFCl}_{3}\left({ }^{19} \mathrm{~F}\right)$.

* N.B. Compound $\mathbf{4}$ induces hydrodefluorination of $\mathbf{2 a}$ and $\mathbf{2 b}$ at $160^{\circ} \mathrm{C}$ in the absence of $\mathbf{1}$. 


\section{Crystallographic Data}

\section{a) Structural Data for $1 \cdot[\mathrm{F}]\left[\mathrm{C}_{5} \mathrm{~F}_{4} \mathrm{~N}\right]$}

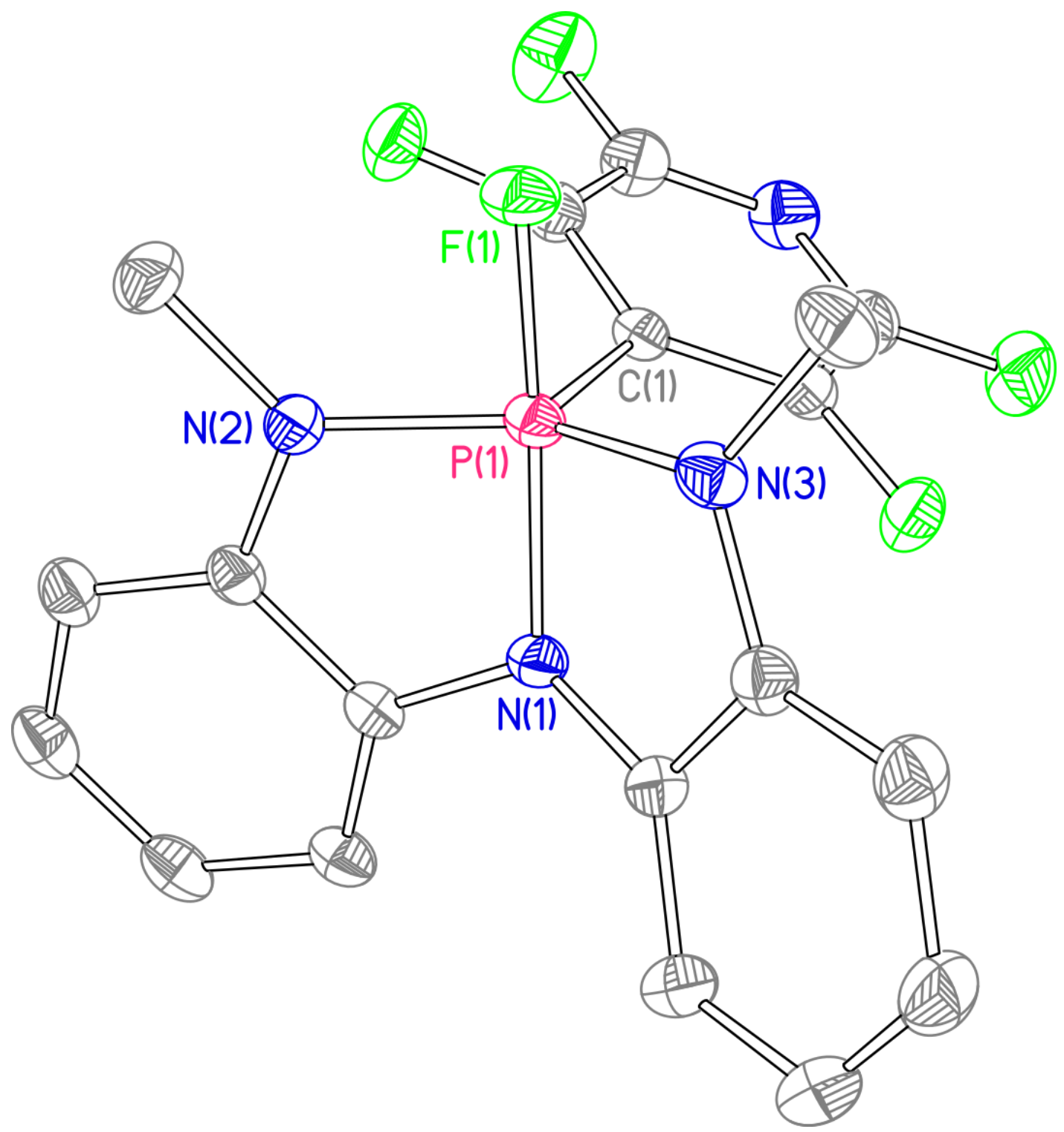

Figure S9. Thermal ellipsoid plot rendered at the $50 \%$ probability level for $\mathbf{1} \cdot[\mathrm{F}]\left[\mathrm{C}_{5} \mathrm{~F}_{4} \mathrm{~N}\right]$. Hydrogen atoms are omitted for clarity. 
Table S1. Crystal data and structure refinement for $\mathbf{1} \cdot[\mathrm{F}]\left[\mathrm{C}_{5} \mathrm{~F}_{4} \mathrm{~N}\right]$.

Identification code

Empirical formula

Formula weight

Temperature

Wavelength

Crystal system

Space group

Unit cell dimensions

Volume

Z

Density (calculated)

Absorption coefficient

$\mathrm{F}(000)$

Crystal size

Theta range for data collection

Index ranges

Reflections collected

Independent reflections

Completeness to theta $=25.242^{\circ}$

Absorption correction

Refinement method

Data / restraints / parameters

Goodness-of-fit on $\mathrm{F}^{2}$

Final R indices [I $>2$ sigma(I)]

$\mathrm{R}$ indices (all data)

Extinction coefficient

Largest diff. peak and hole
NNNPFpyr_3

C19 H14 F5 N4 P

424.31

100(2) K

$0.71073 \AA$

Orthorhombic

Pbca

$\mathrm{a}=16.2015(8) \AA$

$\mathrm{a}=90^{\circ}$.

$\mathrm{b}=6.6505(3) \AA$

$\mathrm{b}=90^{\circ}$.

$c=33.4158(15) \AA$

$\mathrm{g}=90^{\circ}$.
8

$1.566 \mathrm{Mg} / \mathrm{m}^{3}$

$0.216 \mathrm{~mm}^{-1}$

1728

$0.400 \times 0.280 \times 0.180 \mathrm{~mm}^{3}$

1.751 to $36.391^{\circ}$.

$-26<=\mathrm{h}<=27,-10<=\mathrm{k}<=11,-54<=\mathrm{l}<=55$

170421

$8745[\mathrm{R}(\mathrm{int})=0.0924]$

$100.0 \%$

None

Full-matrix least-squares on $\mathrm{F}^{2}$

8745 / 0 / 264

1.035

$\mathrm{R} 1=0.0422, \mathrm{wR} 2=0.1089$

$\mathrm{R} 1=0.0613, \mathrm{wR} 2=0.1219$

$\mathrm{n} / \mathrm{a}$

0.449 and -0.495 e. $\AA^{-3}$ 
Table S2. Atomic coordinates $\left(\times 10^{4}\right)$ and equivalent isotropic displacement parameters $\left(\AA^{2} \times 10^{3}\right)$ for 1. $[\mathrm{F}]\left[\mathrm{C}_{5} \mathrm{~F}_{4} \mathrm{~N}\right] . \mathrm{U}(\mathrm{eq})$ is defined as one third of the trace of the orthogonalized $\mathrm{U}^{\mathrm{ij}}$ tensor.

\begin{tabular}{|c|c|c|c|c|}
\hline & $\mathrm{x}$ & $\mathrm{y}$ & $\mathrm{z}$ & $\mathrm{U}(\mathrm{eq})$ \\
\hline$P(1)$ & $5069(1)$ & $8742(1)$ & $6413(1)$ & $17(1)$ \\
\hline $\mathrm{F}(1)$ & $4569(1)$ & $10467(1)$ & $6660(1)$ & $25(1)$ \\
\hline $\mathrm{F}(2)$ & $4109(1)$ & $7054(1)$ & $5679(1)$ & $28(1)$ \\
\hline $\mathrm{F}(3)$ & $3968(1)$ & $7089(1)$ & $7097(1)$ & $30(1)$ \\
\hline$F(4)$ & $2711(1)$ & $4919(1)$ & $5686(1)$ & $38(1)$ \\
\hline $\mathrm{F}(5)$ & $2615(1)$ & $4829(2)$ & $7034(1)$ & $44(1)$ \\
\hline $\mathrm{N}(1)$ & $5698(1)$ & $7025(1)$ & $6152(1)$ & $18(1)$ \\
\hline $\mathrm{N}(2)$ & $5606(1)$ & $8184(1)$ & $6830(1)$ & $20(1)$ \\
\hline $\mathrm{N}(3)$ & $5126(1)$ & $10266(1)$ & $6015(1)$ & $21(1)$ \\
\hline $\mathrm{N}(4)$ & $2671(1)$ & $4871(2)$ & $6359(1)$ & $28(1)$ \\
\hline$C(1)$ & $4125(1)$ & 7194(1) & $6390(1)$ & $17(1)$ \\
\hline$C(2)$ & $6112(1)$ & $5693(2)$ & $6407(1)$ & $18(1)$ \\
\hline $\mathrm{C}(3)$ & $6051(1)$ & $6379(2)$ & $6802(1)$ & $19(1)$ \\
\hline$C(4)$ & $3770(1)$ & $6581(2)$ & $6032(1)$ & $20(1)$ \\
\hline$C(5)$ & $5989(1)$ & $7730(2)$ & $5783(1)$ & $20(1)$ \\
\hline$C(6)$ & $3050(1)$ & $5462(2)$ & $6034(1)$ & $24(1)$ \\
\hline$C(7)$ & $5641(1)$ & $9615(2)$ & $5702(1)$ & $21(1)$ \\
\hline$C(8)$ & $3707(1)$ & $6577(2)$ & $6732(1)$ & $21(1)$ \\
\hline$C(9)$ & $6523(1)$ & $3914(2)$ & $6318(1)$ & $23(1)$ \\
\hline$C(10)$ & $3000(1)$ & $5422(2)$ & $6700(1)$ & $27(1)$ \\
\hline$C(11)$ & $5593(1)$ & $9211(2)$ & $7216(1)$ & $27(1)$ \\
\hline$C(12)$ & $6403(1)$ & $5304(2)$ & $7114(1)$ & $24(1)$ \\
\hline$C(13)$ & $6883(1)$ & $2841(2)$ & $6634(1)$ & $27(1)$ \\
\hline$C(14)$ & $6825(1)$ & $3527(2)$ & $7024(1)$ & $28(1)$ \\
\hline$C(15)$ & $6548(1)$ & $6867(2)$ & $5520(1)$ & $30(1)$ \\
\hline$C(16)$ & $5825(1)$ & $10637(2)$ & $5352(1)$ & $28(1)$ \\
\hline$C(17)$ & $4633(1)$ & $12078(2)$ & $5947(1)$ & $29(1)$ \\
\hline$C(18)$ & $6734(1)$ & $7914(2)$ & $5167(1)$ & $36(1)$ \\
\hline C(19) & $6375(1)$ & $9745(2)$ & $5083(1)$ & $34(1)$ \\
\hline
\end{tabular}


Table S3. Bond lengths $[\AA ̊]$ and angles $\left[{ }^{\circ}\right]$ for $\mathbf{1} \cdot[\mathrm{F}]\left[\mathrm{C}_{5} \mathrm{~F}_{4} \mathrm{~N}\right]$.

\begin{tabular}{|c|c|}
\hline$P(1)-F(1)$ & $1.6282(6)$ \\
\hline$P(1)-N(3)$ & $1.6753(9)$ \\
\hline$P(1)-N(2)$ & $1.6841(9)$ \\
\hline$P(1)-N(1)$ & $1.7616(8)$ \\
\hline$P(1)-C(1)$ & $1.8456(10)$ \\
\hline$F(2)-C(4)$ & $1.3370(11)$ \\
\hline$F(3)-C(8)$ & $1.3360(11)$ \\
\hline $\mathrm{F}(4)-\mathrm{C}(6)$ & $1.3357(12)$ \\
\hline$F(5)-C(10)$ & $1.3377(13)$ \\
\hline$N(1)-C(2)$ & $1.3991(12)$ \\
\hline$N(1)-C(5)$ & $1.4014(12)$ \\
\hline$N(2)-C(3)$ & $1.4037(13)$ \\
\hline$N(2)-C(11)$ & $1.4617(13)$ \\
\hline $\mathrm{N}(3)-\mathrm{C}(7)$ & $1.4052(13)$ \\
\hline $\mathrm{N}(3)-\mathrm{C}(17)$ & $1.4640(13)$ \\
\hline$N(4)-C(10)$ & $1.3088(15)$ \\
\hline$N(4)-C(6)$ & $1.3091(15)$ \\
\hline$C(1)-C(8)$ & $1.3881(13)$ \\
\hline$C(1)-C(4)$ & $1.3912(13)$ \\
\hline$C(2)-C(9)$ & $1.3894(14)$ \\
\hline$C(2)-C(3)$ & $1.4022(13)$ \\
\hline$C(3)-C(12)$ & $1.3871(13)$ \\
\hline$C(4)-C(6)$ & $1.3835(14)$ \\
\hline$C(5)-C(15)$ & $1.3856(14)$ \\
\hline$C(5)-C(7)$ & $1.4005(14)$ \\
\hline$C(7)-C(16)$ & $1.3857(14)$ \\
\hline$C(8)-C(10)$ & $1.3832(15)$ \\
\hline$C(9)-C(13)$ & $1.4005(15)$ \\
\hline $\mathrm{C}(9)-\mathrm{H}(9)$ & 0.9500 \\
\hline$C(11)-H(11 A)$ & 0.9800 \\
\hline $\mathrm{C}(11)-\mathrm{H}(11 \mathrm{~B})$ & 0.9800 \\
\hline $\mathrm{C}(11)-\mathrm{H}(11 \mathrm{C})$ & 0.9800 \\
\hline$C(12)-C(14)$ & $1.3983(16)$ \\
\hline $\mathrm{C}(12)-\mathrm{H}(12)$ & 0.9500 \\
\hline $\mathrm{C}(13)-\mathrm{C}(14)$ & $1.3828(17)$ \\
\hline
\end{tabular}




\begin{tabular}{|c|c|}
\hline $\mathrm{C}(13)-\mathrm{H}(13)$ & 0.9500 \\
\hline $\mathrm{C}(14)-\mathrm{H}(14)$ & 0.9500 \\
\hline$C(15)-C(18)$ & $1.4035(17)$ \\
\hline $\mathrm{C}(15)-\mathrm{H}(15)$ & 0.9500 \\
\hline$C(16)-C(19)$ & $1.3977(17)$ \\
\hline $\mathrm{C}(16)-\mathrm{H}(16)$ & 0.9500 \\
\hline $\mathrm{C}(17)-\mathrm{H}(17 \mathrm{~A})$ & 0.9800 \\
\hline $\mathrm{C}(17)-\mathrm{H}(17 \mathrm{~B})$ & 0.9800 \\
\hline $\mathrm{C}(17)-\mathrm{H}(17 \mathrm{C})$ & 0.9800 \\
\hline$C(18)-C(19)$ & $1.378(2)$ \\
\hline $\mathrm{C}(18)-\mathrm{H}(18)$ & 0.9500 \\
\hline $\mathrm{C}(19)-\mathrm{H}(19)$ & 0.9500 \\
\hline$F(1)-P(1)-N(3)$ & $90.22(4)$ \\
\hline$F(1)-P(1)-N(2)$ & $89.59(4)$ \\
\hline$N(3)-P(1)-N(2)$ & $139.66(5)$ \\
\hline$F(1)-P(1)-N(1)$ & $174.28(4)$ \\
\hline$N(3)-P(1)-N(1)$ & $88.12(4)$ \\
\hline$N(2)-P(1)-N(1)$ & $88.14(4)$ \\
\hline$F(1)-P(1)-C(1)$ & $90.09(4)$ \\
\hline$N(3)-P(1)-C(1)$ & $110.58(4)$ \\
\hline$N(2)-P(1)-C(1)$ & $109.76(4)$ \\
\hline$N(1)-P(1)-C(1)$ & $95.62(4)$ \\
\hline$C(2)-N(1)-C(5)$ & $125.86(8)$ \\
\hline$C(2)-N(1)-P(1)$ & $112.78(6)$ \\
\hline$C(5)-N(1)-P(1)$ & 114.41(7) \\
\hline$C(3)-N(2)-C(11)$ & $117.70(8)$ \\
\hline$C(3)-N(2)-P(1)$ & $113.57(6)$ \\
\hline $\mathrm{C}(11)-\mathrm{N}(2)-\mathrm{P}(1)$ & 128.41(7) \\
\hline $\mathrm{C}(7)-\mathrm{N}(3)-\mathrm{C}(17)$ & $117.63(9)$ \\
\hline$C(7)-N(3)-P(1)$ & $115.89(7)$ \\
\hline $\mathrm{C}(17)-\mathrm{N}(3)-\mathrm{P}(1)$ & $126.17(8)$ \\
\hline$C(10)-N(4)-C(6)$ & $116.55(10)$ \\
\hline$C(8)-C(1)-C(4)$ & $114.80(9)$ \\
\hline$C(8)-C(1)-P(1)$ & $122.43(7)$ \\
\hline$C(4)-C(1)-P(1)$ & $122.76(7)$ \\
\hline$C(9)-C(2)-N(1)$ & $129.75(9)$ \\
\hline
\end{tabular}




\begin{tabular}{|c|c|}
\hline$C(9)-C(2)-C(3)$ & $120.75(9)$ \\
\hline$N(1)-C(2)-C(3)$ & $109.48(8)$ \\
\hline $\mathrm{C}(12)-\mathrm{C}(3)-\mathrm{C}(2)$ & $120.81(9)$ \\
\hline $\mathrm{C}(12)-\mathrm{C}(3)-\mathrm{N}(2)$ & $127.08(9)$ \\
\hline$C(2)-C(3)-N(2)$ & $112.11(8)$ \\
\hline$F(2)-C(4)-C(6)$ & $118.57(9)$ \\
\hline$F(2)-C(4)-C(1)$ & $121.34(9)$ \\
\hline$C(6)-C(4)-C(1)$ & $120.10(9)$ \\
\hline$C(15)-C(5)-C(7)$ & $120.75(9)$ \\
\hline$C(15)-C(5)-N(1)$ & $129.73(10)$ \\
\hline $\mathrm{C}(7)-\mathrm{C}(5)-\mathrm{N}(1)$ & $109.49(8)$ \\
\hline$N(4)-C(6)-F(4)$ & $116.68(10)$ \\
\hline$N(4)-C(6)-C(4)$ & $124.16(9)$ \\
\hline$F(4)-C(6)-C(4)$ & $119.16(10)$ \\
\hline$C(16)-C(7)-C(5)$ & $121.05(10)$ \\
\hline$C(16)-C(7)-N(3)$ & $127.12(10)$ \\
\hline $\mathrm{C}(5)-\mathrm{C}(7)-\mathrm{N}(3)$ & $111.82(8)$ \\
\hline$F(3)-C(8)-C(10)$ & $118.31(9)$ \\
\hline$F(3)-C(8)-C(1)$ & $121.36(9)$ \\
\hline $\mathrm{C}(10)-\mathrm{C}(8)-\mathrm{C}(1)$ & $120.33(9)$ \\
\hline$C(2)-C(9)-C(13)$ & $118.23(10)$ \\
\hline$C(2)-C(9)-H(9)$ & 120.9 \\
\hline $\mathrm{C}(13)-\mathrm{C}(9)-\mathrm{H}(9)$ & 120.9 \\
\hline$N(4)-C(10)-F(5)$ & $116.92(10)$ \\
\hline $\mathrm{N}(4)-\mathrm{C}(10)-\mathrm{C}(8)$ & $124.03(10)$ \\
\hline$F(5)-C(10)-C(8)$ & $119.05(10)$ \\
\hline $\mathrm{N}(2)-\mathrm{C}(11)-\mathrm{H}(11 \mathrm{~A})$ & 109.5 \\
\hline $\mathrm{N}(2)-\mathrm{C}(11)-\mathrm{H}(11 \mathrm{~B})$ & 109.5 \\
\hline$H(11 A)-C(11)-H(11 B)$ & 109.5 \\
\hline $\mathrm{N}(2)-\mathrm{C}(11)-\mathrm{H}(11 \mathrm{C})$ & 109.5 \\
\hline $\mathrm{H}(11 \mathrm{~A})-\mathrm{C}(11)-\mathrm{H}(11 \mathrm{C})$ & 109.5 \\
\hline $\mathrm{H}(11 \mathrm{~B})-\mathrm{C}(11)-\mathrm{H}(11 \mathrm{C})$ & 109.5 \\
\hline$C(3)-C(12)-C(14)$ & $118.25(10)$ \\
\hline $\mathrm{C}(3)-\mathrm{C}(12)-\mathrm{H}(12)$ & 120.9 \\
\hline $\mathrm{C}(14)-\mathrm{C}(12)-\mathrm{H}(12)$ & 120.9 \\
\hline$C(14)-C(13)-C(9)$ & $120.87(10)$ \\
\hline $\mathrm{C}(14)-\mathrm{C}(13)-\mathrm{H}(13)$ & 119.6 \\
\hline
\end{tabular}




\begin{tabular}{ll}
$\mathrm{C}(9)-\mathrm{C}(13)-\mathrm{H}(13)$ & 119.6 \\
$\mathrm{C}(13)-\mathrm{C}(14)-\mathrm{C}(12)$ & $121.08(10)$ \\
$\mathrm{C}(13)-\mathrm{C}(14)-\mathrm{H}(14)$ & 119.5 \\
$\mathrm{C}(12)-\mathrm{C}(14)-\mathrm{H}(14)$ & 119.5 \\
$\mathrm{C}(5)-\mathrm{C}(15)-\mathrm{C}(18)$ & $117.91(11)$ \\
$\mathrm{C}(5)-\mathrm{C}(15)-\mathrm{H}(15)$ & 121.0 \\
$\mathrm{C}(18)-\mathrm{C}(15)-\mathrm{H}(15)$ & 121.0 \\
$\mathrm{C}(7)-\mathrm{C}(16)-\mathrm{C}(19)$ & $118.09(11)$ \\
$\mathrm{C}(7)-\mathrm{C}(16)-\mathrm{H}(16)$ & 121.0 \\
$\mathrm{C}(19)-\mathrm{C}(16)-\mathrm{H}(16)$ & 121.0 \\
$\mathrm{~N}(3)-\mathrm{C}(17)-\mathrm{H}(17 \mathrm{~A})$ & 109.5 \\
$\mathrm{~N}(3)-\mathrm{C}(17)-\mathrm{H}(17 \mathrm{~B})$ & 109.5 \\
$\mathrm{H}(17 \mathrm{~A})-\mathrm{C}(17)-\mathrm{H}(17 \mathrm{~B})$ & 109.5 \\
$\mathrm{~N}(3)-\mathrm{C}(17)-\mathrm{H}(17 \mathrm{C})$ & 109.5 \\
$\mathrm{H}(17 \mathrm{~A})-\mathrm{C}(17)-\mathrm{H}(17 \mathrm{C})$ & 109.5 \\
$\mathrm{H}(17 \mathrm{~B})-\mathrm{C}(17)-\mathrm{H}(17 \mathrm{C})$ & 109.5 \\
$\mathrm{C}(19)-\mathrm{C}(18)-\mathrm{C}(15)$ & $121.22(11)$ \\
$\mathrm{C}(19)-\mathrm{C}(18)-\mathrm{H}(18)$ & 119.4 \\
$\mathrm{C}(15)-\mathrm{C}(18)-\mathrm{H}(18)$ & 119.4 \\
$\mathrm{C}(18)-\mathrm{C}(19)-\mathrm{C}(16)$ & $120.94(11)$ \\
$\mathrm{C}(18)-\mathrm{C}(19)-\mathrm{H}(19)$ & 119.5 \\
$\mathrm{C}(16)-\mathrm{C}(19)-\mathrm{H}(19)$ & 119.5 \\
\hline
\end{tabular}

Symmetry transformations used to generate equivalent atoms: 
Table S4. Anisotropic displacement parameters $\left(\AA^{2} \times 10^{3}\right)$ for $\mathbf{1} \cdot[\mathrm{F}]\left[\mathrm{C}_{5} \mathrm{~F}_{4} \mathrm{~N}\right]$. The anisotropic displacement factor exponent takes the form: $-2 p^{2}\left[h^{2} a^{* 2} U^{11}+\ldots+2 h k a * b^{*} U^{12}\right]$

\begin{tabular}{|c|c|c|c|c|c|c|}
\hline & $\mathrm{U}^{11}$ & $\mathrm{U}^{22}$ & $\mathrm{U}^{33}$ & $\mathrm{U}^{23}$ & $\mathrm{U}^{13}$ & $\mathrm{U}^{12}$ \\
\hline$P(1)$ & $16(1)$ & $15(1)$ & $20(1)$ & $-1(1)$ & $1(1)$ & $1(1)$ \\
\hline $\mathrm{F}(1)$ & $26(1)$ & $20(1)$ & $28(1)$ & $-5(1)$ & $3(1)$ & $5(1)$ \\
\hline$F(2)$ & $29(1)$ & $36(1)$ & $18(1)$ & $0(1)$ & $0(1)$ & $-5(1)$ \\
\hline $\mathrm{F}(3)$ & $28(1)$ & $43(1)$ & $19(1)$ & $1(1)$ & $0(1)$ & $-8(1)$ \\
\hline$F(4)$ & $34(1)$ & $47(1)$ & $32(1)$ & $-9(1)$ & $-10(1)$ & $-11(1)$ \\
\hline $\mathrm{F}(5)$ & $39(1)$ & $59(1)$ & $33(1)$ & $5(1)$ & $9(1)$ & $-23(1)$ \\
\hline $\mathrm{N}(1)$ & $18(1)$ & $18(1)$ & $19(1)$ & $0(1)$ & $2(1)$ & $4(1)$ \\
\hline $\mathrm{N}(2)$ & $19(1)$ & $22(1)$ & $20(1)$ & $-4(1)$ & $-1(1)$ & $1(1)$ \\
\hline $\mathrm{N}(3)$ & $22(1)$ & $17(1)$ & $25(1)$ & $2(1)$ & $1(1)$ & $2(1)$ \\
\hline $\mathrm{N}(4)$ & $22(1)$ & $29(1)$ & $34(1)$ & $-2(1)$ & $0(1)$ & $-6(1)$ \\
\hline$C(1)$ & $15(1)$ & $17(1)$ & $20(1)$ & $0(1)$ & $0(1)$ & $2(1)$ \\
\hline$C(2)$ & $15(1)$ & $18(1)$ & $22(1)$ & $1(1)$ & $0(1)$ & $1(1)$ \\
\hline$C(3)$ & $14(1)$ & $21(1)$ & $22(1)$ & $0(1)$ & $-1(1)$ & $-1(1)$ \\
\hline$C(4)$ & $18(1)$ & $20(1)$ & $21(1)$ & $-1(1)$ & $-1(1)$ & $1(1)$ \\
\hline$C(5)$ & $19(1)$ & $23(1)$ & $19(1)$ & $-1(1)$ & $1(1)$ & $1(1)$ \\
\hline$C(6)$ & $21(1)$ & $24(1)$ & $27(1)$ & $-4(1)$ & $-4(1)$ & $-1(1)$ \\
\hline $\mathrm{C}(7)$ & $19(1)$ & $22(1)$ & $22(1)$ & $2(1)$ & $0(1)$ & $-2(1)$ \\
\hline$C(8)$ & $19(1)$ & $25(1)$ & $21(1)$ & $1(1)$ & $0(1)$ & $-1(1)$ \\
\hline $\mathrm{C}(9)$ & $19(1)$ & $20(1)$ & $30(1)$ & $-2(1)$ & $0(1)$ & $3(1)$ \\
\hline$C(10)$ & $23(1)$ & $30(1)$ & $28(1)$ & $2(1)$ & $3(1)$ & $-6(1)$ \\
\hline $\mathrm{C}(11)$ & $27(1)$ & $32(1)$ & $22(1)$ & $-8(1)$ & $-1(1)$ & $-2(1)$ \\
\hline $\mathrm{C}(12)$ & $19(1)$ & $29(1)$ & $24(1)$ & $5(1)$ & $-2(1)$ & $-1(1)$ \\
\hline $\mathrm{C}(13)$ & $20(1)$ & $22(1)$ & $38(1)$ & $4(1)$ & $-1(1)$ & $4(1)$ \\
\hline$C(14)$ & $20(1)$ & $29(1)$ & $33(1)$ & $10(1)$ & $-3(1)$ & $1(1)$ \\
\hline$C(15)$ & $28(1)$ & $36(1)$ & $24(1)$ & $-1(1)$ & $6(1)$ & $8(1)$ \\
\hline$C(16)$ & $27(1)$ & $31(1)$ & $26(1)$ & $8(1)$ & $0(1)$ & $-3(1)$ \\
\hline $\mathrm{C}(17)$ & $30(1)$ & $22(1)$ & $36(1)$ & $4(1)$ & $-2(1)$ & $7(1)$ \\
\hline $\mathrm{C}(18)$ & $33(1)$ & $51(1)$ & $24(1)$ & $2(1)$ & $9(1)$ & $6(1)$ \\
\hline $\mathrm{C}(19)$ & $32(1)$ & $48(1)$ & $23(1)$ & $8(1)$ & $4(1)$ & $-3(1)$ \\
\hline
\end{tabular}


Table S5. Hydrogen coordinates $\left(\times 10^{4}\right)$ and isotropic displacement parameters $\left(\AA^{2} \times 10^{3}\right)$ for $\mathbf{1} \cdot[\mathrm{F}]\left[\mathrm{C}_{5} \mathrm{~F}_{4} \mathrm{~N}\right]$.

\begin{tabular}{|c|c|c|c|c|}
\hline & $\mathrm{x}$ & $\mathrm{y}$ & $\mathrm{z}$ & $\mathrm{U}(\mathrm{eq})$ \\
\hline $\mathrm{H}(9)$ & 6559 & 3437 & 6051 & 27 \\
\hline $\mathrm{H}(11 \mathrm{~A})$ & 5233 & 8480 & 7402 & 41 \\
\hline $\mathrm{H}(11 \mathrm{~B})$ & 5385 & 10583 & 7181 & 41 \\
\hline $\mathrm{H}(11 \mathrm{C})$ & 6154 & 9260 & 7326 & 41 \\
\hline $\mathrm{H}(12)$ & 6359 & 5763 & 7383 & 29 \\
\hline $\mathrm{H}(13)$ & 7170 & 1627 & 6580 & 32 \\
\hline $\mathrm{H}(14)$ & 7076 & 2779 & 7233 & 33 \\
\hline $\mathrm{H}(15)$ & 6797 & 5606 & 5577 & 35 \\
\hline $\mathrm{H}(16)$ & 5584 & 11909 & 5296 & 34 \\
\hline $\mathrm{H}(17 \mathrm{~A})$ & 4991 & 13264 & 5957 & 44 \\
\hline $\mathrm{H}(17 \mathrm{~B})$ & 4209 & 12186 & 6155 & 44 \\
\hline $\mathrm{H}(17 \mathrm{C})$ & 4367 & 11999 & 5684 & 44 \\
\hline $\mathrm{H}(18)$ & 7114 & 7349 & 4982 & 44 \\
\hline $\mathrm{H}(19)$ & 6503 & 10410 & 4839 & 41 \\
\hline
\end{tabular}


b) Structural Data for $1 \cdot[F]\left[C_{6} F_{4} C F_{3}\right]$

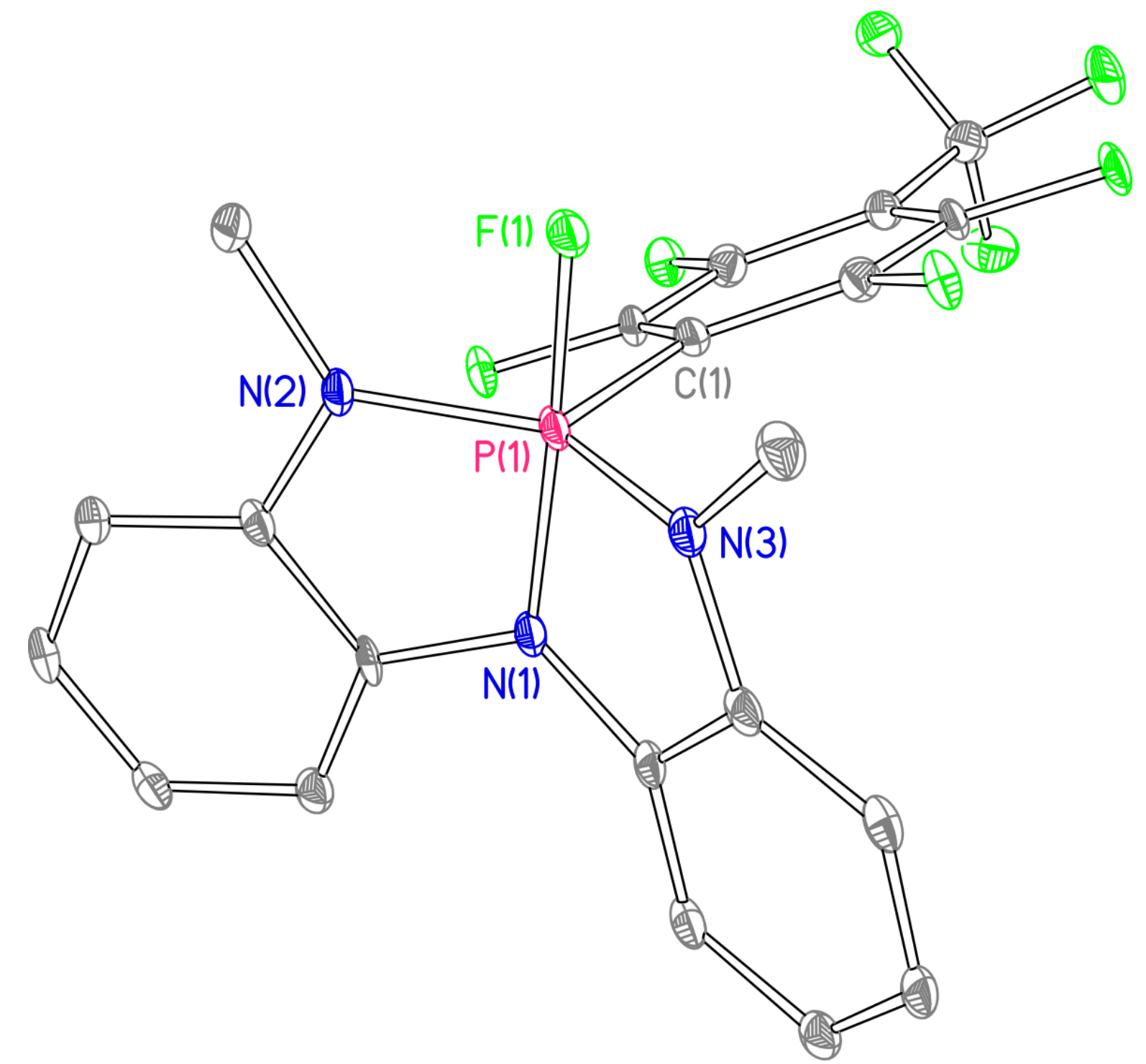

Figure S10. Thermal ellipsoid plot rendered at the $50 \%$ probability level for $\mathbf{1} \cdot[\mathrm{F}]\left[\mathrm{C}_{6} \mathrm{~F}_{4} \mathrm{CF}_{3}\right]$. Hydrogen atoms are omitted for clarity. 
Table S6. Crystal data and structure refinement for $\mathbf{1} \cdot[\mathrm{F}]\left[\mathrm{C}_{6} \mathrm{~F}_{4} \mathrm{CF}_{3}\right]$.

Identification code

Empirical formula

Formula weight

Temperature

Wavelength

Crystal system

Space group

Unit cell dimensions

Volume

Z

Density (calculated)

Absorption coefficient

$\mathrm{F}(000)$

Crystal size

Theta range for data collection

Index ranges

Reflections collected

Independent reflections

Completeness to theta $=8.505^{\circ}$

Absorption correction

Refinement method

Data / restraints / parameters

Goodness-of-fit on $\mathrm{F}^{2}$

Final R indices [I $>2$ sigma(I)]

$\mathrm{R}$ indices (all data)

Extinction coefficient

Largest diff. peak and hole
SL1-051

C21 H14 F8 N3 P

491.32

288(2) K

$0.24797 \AA$

Monoclinic

$\mathrm{P} 21 / \mathrm{c}$

$\mathrm{a}=14.4123(14) \AA$

$\mathrm{a}=90^{\circ}$.

$\mathrm{b}=15.9030(16) \AA$

$\mathrm{b}=92.990(2)^{\circ}$.

$\mathrm{c}=8.3848(8) \AA$

$\mathrm{g}=90^{\circ}$.

1919.2(3) $\AA^{3}$

4

$1.700 \mathrm{Mg} / \mathrm{m}^{3}$

$0.039 \mathrm{~mm}^{-1}$

992

$0.300 \times 0.100 \times 0.080 \mathrm{~mm}^{3}$

0.893 to $8.505^{\circ}$.

$-17<=\mathrm{h}<=17,-18<=\mathrm{k}<=18,-9<=\mathrm{l}<=9$

40971

$3385[\mathrm{R}(\mathrm{int})=0.0621]$

$99.5 \%$

None

Full-matrix least-squares on $\mathrm{F}^{2}$

3385 / 0 / 300

1.079

$\mathrm{R} 1=0.0340, \mathrm{wR} 2=0.0913$

$\mathrm{R} 1=0.0379, \mathrm{wR} 2=0.0934$

$\mathrm{n} / \mathrm{a}$

0.302 and -0.402 e. $\AA^{-3}$ 
Table S7. Atomic coordinates $\left(\times 10^{4}\right)$ and equivalent isotropic displacement parameters $\left(\AA^{2} \times 10^{3}\right)$ for 1. $[\mathrm{F}]\left[\mathrm{C}_{6} \mathrm{~F}_{4} \mathrm{CF}_{3}\right]$. U(eq) is defined as one third of the trace of the orthogonalized $\mathrm{U}^{\mathrm{ij}}$ tensor.

\begin{tabular}{|c|c|c|c|c|}
\hline & $\mathrm{x}$ & $\mathrm{y}$ & $\mathrm{z}$ & $\mathrm{U}(\mathrm{eq})$ \\
\hline$P(1)$ & $6996(1)$ & $4323(1)$ & $6045(1)$ & $10(1)$ \\
\hline$F(1)$ & $7210(1)$ & $4143(1)$ & $7941(1)$ & $13(1)$ \\
\hline$F(2)$ & $8806(1)$ & $4313(1)$ & $4186(1)$ & $14(1)$ \\
\hline $\mathrm{F}(3)$ & $9879(1)$ & $3036(1)$ & $3478(1)$ & $15(1)$ \\
\hline$F(4)$ & $7910(1)$ & $1184(1)$ & $6182(1)$ & $15(1)$ \\
\hline$F(5)$ & $6863(1)$ & $2444(1)$ & $6903(1)$ & $14(1)$ \\
\hline$F(6)$ & $9438(1)$ & $659(1)$ & $5220(1)$ & $20(1)$ \\
\hline $\mathrm{F}(7)$ & 10501(1) & 1598(1) & $4996(1)$ & $16(1)$ \\
\hline $\mathrm{F}(8)$ & $9655(1)$ & $1216(1)$ & $2922(1)$ & $21(1)$ \\
\hline $\mathrm{N}(1)$ & $6699(1)$ & $4588(1)$ & $4042(2)$ & $11(1)$ \\
\hline $\mathrm{N}(2)$ & $7574(1)$ & $5239(1)$ & $6214(2)$ & $12(1)$ \\
\hline $\mathrm{N}(3)$ & $5900(1)$ & $3978(1)$ & $6194(2)$ & $11(1)$ \\
\hline$C(1)$ & $8136(1)$ & $5557(1)$ & $7603(2)$ & $14(1)$ \\
\hline$C(2)$ & $5452(1)$ & $3653(1)$ & $7611(2)$ & $14(1)$ \\
\hline$C(3)$ & $7612(1)$ & $5711(1)$ & $4800(2)$ & $11(1)$ \\
\hline$C(4)$ & $7106(1)$ & $5329(1)$ & $3515(2)$ & $11(1)$ \\
\hline$C(5)$ & $7070(1)$ & $5698(1)$ & $2014(2)$ & $12(1)$ \\
\hline$C(6)$ & $7530(1)$ & $6463(1)$ & $1818(2)$ & $14(1)$ \\
\hline$C(7)$ & $8032(1)$ & $6835(1)$ & $3086(2)$ & $14(1)$ \\
\hline$C(8)$ & $8078(1)$ & $6461(1)$ & $4594(2)$ & $13(1)$ \\
\hline$C(9)$ & $5394(1)$ & $3882(1)$ & $4720(2)$ & $12(1)$ \\
\hline$C(10)$ & $4544(1)$ & $3483(1)$ & $4465(2)$ & $13(1)$ \\
\hline$C(11)$ & $4164(1)$ & $3432(1)$ & $2900(2)$ & $13(1)$ \\
\hline$C(12)$ & $4624(1)$ & $3776(1)$ & $1641(2)$ & $14(1)$ \\
\hline$C(13)$ & $5481(1)$ & 4181(1) & $1905(2)$ & $13(1)$ \\
\hline$C(14)$ & $5861(1)$ & $4232(1)$ & $3455(2)$ & $11(1)$ \\
\hline$C(15)$ & $7772(1)$ & $3439(1)$ & $5557(2)$ & $11(1)$ \\
\hline$C(16)$ & $8559(1)$ & $3547(1)$ & $4687(2)$ & $12(1)$ \\
\hline $\mathrm{C}(17)$ & $9130(1)$ & $2883(1)$ & $4329(2)$ & $12(1)$ \\
\hline$C(18)$ & $8954(1)$ & $2066(1)$ & $4828(2)$ & $13(1)$ \\
\hline$C(19)$ & $8157(1)$ & 1946(1) & $5667(2)$ & $12(1)$ \\
\hline$C(20)$ & $7598(1)$ & $2619(1)$ & $6031(2)$ & $12(1)$ \\
\hline
\end{tabular}


Table S8. Bond lengths $[\AA]$ and angles $\left[^{\circ}\right]$ for $\mathbf{1} \cdot[\mathrm{F}]\left[\mathrm{C}_{6} \mathrm{~F}_{4} \mathrm{CF}_{3}\right]$.

\begin{tabular}{|c|c|}
\hline$P(1)-F(1)$ & $1.6290(10)$ \\
\hline$P(1)-N(2)$ & $1.6798(15)$ \\
\hline$P(1)-N(3)$ & $1.6829(16)$ \\
\hline$P(1)-N(1)$ & $1.7621(14)$ \\
\hline$P(1)-C(15)$ & $1.8551(18)$ \\
\hline$F(2)-C(16)$ & $1.343(2)$ \\
\hline $\mathrm{F}(3)-\mathrm{C}(17)$ & $1.347(2)$ \\
\hline $\mathrm{F}(4)-\mathrm{C}(19)$ & $1.341(2)$ \\
\hline$F(5)-C(20)$ & $1.347(2)$ \\
\hline$F(6)-C(21)$ & $1.330(2)$ \\
\hline $\mathrm{F}(7)-\mathrm{C}(21)$ & $1.345(2)$ \\
\hline $\mathrm{F}(8)-\mathrm{C}(21)$ & $1.340(2)$ \\
\hline$N(1)-C(4)$ & $1.399(2)$ \\
\hline$N(1)-C(14)$ & $1.400(2)$ \\
\hline $\mathrm{N}(2)-\mathrm{C}(3)$ & $1.407(2)$ \\
\hline$N(2)-C(1)$ & $1.473(2)$ \\
\hline$N(3)-C(9)$ & $1.410(2)$ \\
\hline $\mathrm{N}(3)-\mathrm{C}(2)$ & $1.474(2)$ \\
\hline $\mathrm{C}(1)-\mathrm{H}(1 \mathrm{~A})$ & 0.9600 \\
\hline $\mathrm{C}(1)-\mathrm{H}(1 \mathrm{~B})$ & 0.9600 \\
\hline $\mathrm{C}(1)-\mathrm{H}(1 \mathrm{C})$ & 0.9600 \\
\hline $\mathrm{C}(2)-\mathrm{H}(2 \mathrm{~A})$ & 0.9600 \\
\hline $\mathrm{C}(2)-\mathrm{H}(2 \mathrm{~B})$ & 0.9600 \\
\hline $\mathrm{C}(2)-\mathrm{H}(2 \mathrm{C})$ & 0.9600 \\
\hline$C(3)-C(8)$ & $1.385(3)$ \\
\hline$C(3)-C(4)$ & $1.406(2)$ \\
\hline$C(4)-C(5)$ & $1.387(2)$ \\
\hline$C(5)-C(6)$ & $1.400(3)$ \\
\hline $\mathrm{C}(5)-\mathrm{H}(5)$ & 0.9300 \\
\hline$C(6)-C(7)$ & $1.386(3)$ \\
\hline$C(6)-H(6)$ & 0.9300 \\
\hline$C(7)-C(8)$ & $1.396(3)$ \\
\hline $\mathrm{C}(7)-\mathrm{H}(7)$ & 0.9300 \\
\hline $\mathrm{C}(8)-\mathrm{H}(8)$ & 0.9300 \\
\hline$C(9)-C(10)$ & $1.386(3)$ \\
\hline
\end{tabular}




\begin{tabular}{|c|c|}
\hline$C(9)-C(14)$ & $1.401(3)$ \\
\hline$C(10)-C(11)$ & $1.398(2)$ \\
\hline $\mathrm{C}(10)-\mathrm{H}(10)$ & 0.9300 \\
\hline $\mathrm{C}(11)-\mathrm{C}(12)$ & $1.388(3)$ \\
\hline $\mathrm{C}(11)-\mathrm{H}(11)$ & 0.9300 \\
\hline$C(12)-C(13)$ & $1.401(3)$ \\
\hline $\mathrm{C}(12)-\mathrm{H}(12)$ & 0.9300 \\
\hline$C(13)-C(14)$ & $1.386(2)$ \\
\hline $\mathrm{C}(13)-\mathrm{H}(13)$ & 0.9300 \\
\hline$C(15)-C(20)$ & $1.389(2)$ \\
\hline$C(15)-C(16)$ & $1.392(3)$ \\
\hline$C(16)-C(17)$ & $1.382(3)$ \\
\hline $\mathrm{C}(17)-\mathrm{C}(18)$ & $1.392(3)$ \\
\hline $\mathrm{C}(18)-\mathrm{C}(19)$ & $1.390(3)$ \\
\hline$C(18)-C(21)$ & $1.510(3)$ \\
\hline$C(19)-C(20)$ & $1.383(3)$ \\
\hline$F(1)-P(1)-N(2)$ & $90.13(6)$ \\
\hline $\mathrm{F}(1)-\mathrm{P}(1)-\mathrm{N}(3)$ & $90.02(6)$ \\
\hline$N(2)-P(1)-N(3)$ & $137.66(8)$ \\
\hline$F(1)-P(1)-N(1)$ & $175.02(7)$ \\
\hline$N(2)-P(1)-N(1)$ & $88.16(7)$ \\
\hline$N(3)-P(1)-N(1)$ & $88.09(7)$ \\
\hline $\mathrm{F}(1)-\mathrm{P}(1)-\mathrm{C}(15)$ & $89.80(6)$ \\
\hline$N(2)-P(1)-C(15)$ & $111.98(8)$ \\
\hline$N(3)-P(1)-C(15)$ & $110.35(8)$ \\
\hline$N(1)-P(1)-C(15)$ & $95.18(7)$ \\
\hline$C(4)-N(1)-C(14)$ & $126.69(14)$ \\
\hline$C(4)-N(1)-P(1)$ & $114.68(11)$ \\
\hline$C(14)-N(1)-P(1)$ & $113.64(12)$ \\
\hline$C(3)-N(2)-C(1)$ & $116.05(14)$ \\
\hline$C(3)-N(2)-P(1)$ & $115.61(12)$ \\
\hline$C(1)-N(2)-P(1)$ & $127.89(12)$ \\
\hline$C(9)-N(3)-C(2)$ & $116.10(15)$ \\
\hline$C(9)-N(3)-P(1)$ & $114.49(12)$ \\
\hline$C(2)-N(3)-P(1)$ & $128.77(12)$ \\
\hline $\mathrm{N}(2)-\mathrm{C}(1)-\mathrm{H}(1 \mathrm{~A})$ & 109.5 \\
\hline
\end{tabular}




\begin{tabular}{|c|c|}
\hline $\mathrm{N}(2)-\mathrm{C}(1)-\mathrm{H}(1 \mathrm{~B})$ & 109.5 \\
\hline $\mathrm{H}(1 \mathrm{~A})-\mathrm{C}(1)-\mathrm{H}(1 \mathrm{~B})$ & 109.5 \\
\hline $\mathrm{N}(2)-\mathrm{C}(1)-\mathrm{H}(1 \mathrm{C})$ & 109.5 \\
\hline $\mathrm{H}(1 \mathrm{~A})-\mathrm{C}(1)-\mathrm{H}(1 \mathrm{C})$ & 109.5 \\
\hline $\mathrm{H}(1 \mathrm{~B})-\mathrm{C}(1)-\mathrm{H}(1 \mathrm{C})$ & 109.5 \\
\hline $\mathrm{N}(3)-\mathrm{C}(2)-\mathrm{H}(2 \mathrm{~A})$ & 109.5 \\
\hline $\mathrm{N}(3)-\mathrm{C}(2)-\mathrm{H}(2 \mathrm{~B})$ & 109.5 \\
\hline $\mathrm{H}(2 \mathrm{~A})-\mathrm{C}(2)-\mathrm{H}(2 \mathrm{~B})$ & 109.5 \\
\hline $\mathrm{N}(3)-\mathrm{C}(2)-\mathrm{H}(2 \mathrm{C})$ & 109.5 \\
\hline $\mathrm{H}(2 \mathrm{~A})-\mathrm{C}(2)-\mathrm{H}(2 \mathrm{C})$ & 109.5 \\
\hline $\mathrm{H}(2 \mathrm{~B})-\mathrm{C}(2)-\mathrm{H}(2 \mathrm{C})$ & 109.5 \\
\hline$C(8)-C(3)-C(4)$ & $120.74(16)$ \\
\hline $\mathrm{C}(8)-\mathrm{C}(3)-\mathrm{N}(2)$ & $127.28(16)$ \\
\hline$C(4)-C(3)-N(2)$ & $111.98(15)$ \\
\hline$C(5)-C(4)-N(1)$ & $130.24(16)$ \\
\hline$C(5)-C(4)-C(3)$ & $120.46(16)$ \\
\hline$N(1)-C(4)-C(3)$ & $109.30(15)$ \\
\hline$C(4)-C(5)-C(6)$ & $118.54(16)$ \\
\hline $\mathrm{C}(4)-\mathrm{C}(5)-\mathrm{H}(5)$ & 120.7 \\
\hline $\mathrm{C}(6)-\mathrm{C}(5)-\mathrm{H}(5)$ & 120.7 \\
\hline$C(7)-C(6)-C(5)$ & $120.82(16)$ \\
\hline$C(7)-C(6)-H(6)$ & 119.6 \\
\hline $\mathrm{C}(5)-\mathrm{C}(6)-\mathrm{H}(6)$ & 119.6 \\
\hline$C(6)-C(7)-C(8)$ & $120.76(17)$ \\
\hline $\mathrm{C}(6)-\mathrm{C}(7)-\mathrm{H}(7)$ & 119.6 \\
\hline $\mathrm{C}(8)-\mathrm{C}(7)-\mathrm{H}(7)$ & 119.6 \\
\hline$C(3)-C(8)-C(7)$ & $118.66(16)$ \\
\hline $\mathrm{C}(3)-\mathrm{C}(8)-\mathrm{H}(8)$ & 120.7 \\
\hline $\mathrm{C}(7)-\mathrm{C}(8)-\mathrm{H}(8)$ & 120.7 \\
\hline $\mathrm{C}(10)-\mathrm{C}(9)-\mathrm{C}(14)$ & $121.34(16)$ \\
\hline$C(10)-C(9)-N(3)$ & $126.68(16)$ \\
\hline$C(14)-C(9)-N(3)$ & $111.97(16)$ \\
\hline$C(9)-C(10)-C(11)$ & $118.15(16)$ \\
\hline $\mathrm{C}(9)-\mathrm{C}(10)-\mathrm{H}(10)$ & 120.9 \\
\hline $\mathrm{C}(11)-\mathrm{C}(10)-\mathrm{H}(10)$ & 120.9 \\
\hline$C(12)-C(11)-C(10)$ & $120.74(17)$ \\
\hline $\mathrm{C}(12)-\mathrm{C}(11)-\mathrm{H}(11)$ & 119.6 \\
\hline
\end{tabular}




\begin{tabular}{|c|c|}
\hline$C(10)-C(11)-H(11)$ & 119.6 \\
\hline$C(11)-C(12)-C(13)$ & $120.91(16)$ \\
\hline $\mathrm{C}(11)-\mathrm{C}(12)-\mathrm{H}(12)$ & 119.5 \\
\hline $\mathrm{C}(13)-\mathrm{C}(12)-\mathrm{H}(12)$ & 119.5 \\
\hline$C(14)-C(13)-C(12)$ & $118.50(16)$ \\
\hline $\mathrm{C}(14)-\mathrm{C}(13)-\mathrm{H}(13)$ & 120.7 \\
\hline $\mathrm{C}(12)-\mathrm{C}(13)-\mathrm{H}(13)$ & 120.7 \\
\hline$C(13)-C(14)-N(1)$ & $130.16(16)$ \\
\hline$C(13)-C(14)-C(9)$ & $120.35(17)$ \\
\hline$N(1)-C(14)-C(9)$ & $109.48(15)$ \\
\hline$C(20)-C(15)-C(16)$ & $115.47(16)$ \\
\hline$C(20)-C(15)-P(1)$ & $121.95(14)$ \\
\hline$C(16)-C(15)-P(1)$ & $122.58(13)$ \\
\hline$F(2)-C(16)-C(17)$ & $117.08(16)$ \\
\hline$F(2)-C(16)-C(15)$ & $120.90(15)$ \\
\hline$C(17)-C(16)-C(15)$ & $122.00(16)$ \\
\hline $\mathrm{F}(3)-\mathrm{C}(17)-\mathrm{C}(16)$ & $118.65(16)$ \\
\hline $\mathrm{F}(3)-\mathrm{C}(17)-\mathrm{C}(18)$ & $119.46(16)$ \\
\hline$C(16)-C(17)-C(18)$ & $121.89(17)$ \\
\hline $\mathrm{C}(19)-\mathrm{C}(18)-\mathrm{C}(17)$ & $116.65(16)$ \\
\hline$C(19)-C(18)-C(21)$ & $123.86(16)$ \\
\hline $\mathrm{C}(17)-\mathrm{C}(18)-\mathrm{C}(21)$ & $119.47(17)$ \\
\hline$F(4)-C(19)-C(20)$ & $117.40(16)$ \\
\hline$F(4)-C(19)-C(18)$ & $121.85(16)$ \\
\hline $\mathrm{C}(20)-\mathrm{C}(19)-\mathrm{C}(18)$ & $120.73(16)$ \\
\hline$F(5)-C(20)-C(19)$ & $116.31(15)$ \\
\hline$F(5)-C(20)-C(15)$ & $120.47(16)$ \\
\hline$C(19)-C(20)-C(15)$ & $123.22(17)$ \\
\hline$F(6)-C(21)-F(8)$ & $107.81(14)$ \\
\hline $\mathrm{F}(6)-\mathrm{C}(21)-\mathrm{F}(7)$ & $107.25(14)$ \\
\hline $\mathrm{F}(8)-\mathrm{C}(21)-\mathrm{F}(7)$ & $106.95(15)$ \\
\hline$F(6)-C(21)-C(18)$ & $112.33(15)$ \\
\hline$F(8)-C(21)-C(18)$ & $111.73(14)$ \\
\hline $\mathrm{F}(7)-\mathrm{C}(21)-\mathrm{C}(18)$ & $110.52(15)$ \\
\hline
\end{tabular}

Symmetry transformations used to generate equivalent atoms: 
Table S9. Anisotropic displacement parameters $\left(\AA^{2} \times 10^{3}\right)$ for $\mathbf{1} \cdot[\mathrm{F}]\left[\mathrm{C}_{6} \mathrm{~F}_{4} \mathrm{CF}_{3}\right]$. The anisotropic displacement factor exponent takes the form: $-2 \mathrm{p}^{2}\left[\mathrm{~h}^{2} \mathrm{a}^{* 2} \mathrm{U}^{11}+\ldots+2 \mathrm{hka} \mathrm{b}^{*} \mathrm{U}^{12}\right]$

\begin{tabular}{|c|c|c|c|c|c|c|}
\hline & $\mathrm{U}^{11}$ & $\mathrm{U}^{22}$ & $\mathrm{U}^{33}$ & $\mathrm{U}^{23}$ & $\mathrm{U}^{13}$ & $\mathrm{U}^{12}$ \\
\hline$P(1)$ & $16(1)$ & $4(1)$ & $10(1)$ & $0(1)$ & $0(1)$ & $0(1)$ \\
\hline $\mathrm{F}(1)$ & $20(1)$ & $8(1)$ & $10(1)$ & $0(1)$ & $0(1)$ & $0(1)$ \\
\hline$F(2)$ & $19(1)$ & $6(1)$ & $17(1)$ & $2(1)$ & $2(1)$ & $-1(1)$ \\
\hline $\mathrm{F}(3)$ & $17(1)$ & $12(1)$ & $17(1)$ & $-1(1)$ & $3(1)$ & $1(1)$ \\
\hline $\mathrm{F}(4)$ & $23(1)$ & $4(1)$ & $17(1)$ & $2(1)$ & $1(1)$ & $0(1)$ \\
\hline$F(5)$ & $19(1)$ & $8(1)$ & $17(1)$ & $3(1)$ & $5(1)$ & $-1(1)$ \\
\hline$F(6)$ & $26(1)$ & $6(1)$ & $28(1)$ & $2(1)$ & $2(1)$ & $4(1)$ \\
\hline$F(7)$ & $17(1)$ & $13(1)$ & $19(1)$ & $-2(1)$ & $-2(1)$ & $4(1)$ \\
\hline$F(8)$ & $27(1)$ & $20(1)$ & $16(1)$ & $-6(1)$ & $-2(1)$ & $8(1)$ \\
\hline $\mathrm{N}(1)$ & $15(1)$ & $6(1)$ & $11(1)$ & $0(1)$ & $0(1)$ & $-1(1)$ \\
\hline $\mathrm{N}(2)$ & $18(1)$ & $6(1)$ & $12(1)$ & $-1(1)$ & $-1(1)$ & $-1(1)$ \\
\hline $\mathrm{N}(3)$ & $17(1)$ & $7(1)$ & $10(1)$ & $0(1)$ & $1(1)$ & $-1(1)$ \\
\hline $\mathrm{C}(1)$ & $21(1)$ & $9(1)$ & $13(1)$ & $-2(1)$ & $-2(1)$ & $-1(1)$ \\
\hline$C(2)$ & $19(1)$ & $12(1)$ & $12(1)$ & $1(1)$ & $3(1)$ & $-1(1)$ \\
\hline$C(3)$ & $15(1)$ & $6(1)$ & $13(1)$ & $0(1)$ & $2(1)$ & $2(1)$ \\
\hline$C(4)$ & $15(1)$ & $3(1)$ & $16(1)$ & $-1(1)$ & $2(1)$ & $2(1)$ \\
\hline$C(5)$ & $16(1)$ & $8(1)$ & $12(1)$ & $-2(1)$ & $1(1)$ & $2(1)$ \\
\hline$C(6)$ & $19(1)$ & $8(1)$ & $14(1)$ & $2(1)$ & $2(1)$ & $3(1)$ \\
\hline $\mathrm{C}(7)$ & $19(1)$ & $5(1)$ & $19(1)$ & $0(1)$ & $4(1)$ & $0(1)$ \\
\hline$C(8)$ & $17(1)$ & $7(1)$ & $16(1)$ & $-2(1)$ & $-1(1)$ & $2(1)$ \\
\hline$C(9)$ & $18(1)$ & $5(1)$ & $12(1)$ & $-1(1)$ & $0(1)$ & $3(1)$ \\
\hline$C(10)$ & $18(1)$ & $6(1)$ & $15(1)$ & $0(1)$ & $3(1)$ & $2(1)$ \\
\hline$C(11)$ & $15(1)$ & $6(1)$ & $20(1)$ & $-1(1)$ & $0(1)$ & $1(1)$ \\
\hline$C(12)$ & $20(1)$ & $8(1)$ & $13(1)$ & $-2(1)$ & $-2(1)$ & $2(1)$ \\
\hline$C(13)$ & $20(1)$ & $6(1)$ & $12(1)$ & $1(1)$ & $2(1)$ & $0(1)$ \\
\hline$C(14)$ & $14(1)$ & $5(1)$ & $15(1)$ & $-1(1)$ & $0(1)$ & $1(1)$ \\
\hline$C(15)$ & $16(1)$ & $7(1)$ & $10(1)$ & $0(1)$ & $-2(1)$ & $0(1)$ \\
\hline$C(16)$ & $18(1)$ & $5(1)$ & $12(1)$ & $1(1)$ & $-3(1)$ & $-2(1)$ \\
\hline $\mathrm{C}(17)$ & $14(1)$ & $11(1)$ & $12(1)$ & $-1(1)$ & $1(1)$ & $-1(1)$ \\
\hline$C(18)$ & $18(1)$ & $8(1)$ & $13(1)$ & $-2(1)$ & $-4(1)$ & $2(1)$ \\
\hline$C(19)$ & $21(1)$ & $5(1)$ & $11(1)$ & $2(1)$ & $-3(1)$ & $-1(1)$ \\
\hline \multirow[t]{2}{*}{$C(20)$} & $15(1)$ & $11(1)$ & $11(1)$ & $0(1)$ & $0(1)$ & $-1(1)$ \\
\hline & & & & S33 & & \\
\hline
\end{tabular}


C(21)

20(1)

12(1)

14(1)

$-2(1)$

$-2(1)$

1(1) 
Table S10. Hydrogen coordinates $\left(\times 10^{4}\right)$ and isotropic displacement parameters $\left(\AA^{2} \times 10^{3}\right)$ for 1•[F] $\left[\mathrm{C}_{6} \mathrm{~F}_{4} \mathrm{CF}_{3}\right]$.

\begin{tabular}{lrrrr}
\hline & \multicolumn{1}{c}{$\mathrm{x}$} & $\mathrm{y}$ & $\mathrm{U}$ & \\
\cline { 1 - 1 } & & & \\
$\mathrm{H}(1 \mathrm{eq})$ & & \\
$\mathrm{H}(1 \mathrm{~B})$ & 8057 & 5196 & 8504 & 22 \\
$\mathrm{H}(1 \mathrm{C})$ & 7940 & 6116 & 7852 & 22 \\
$\mathrm{H}(2 \mathrm{~A})$ & 8780 & 5565 & 7358 & 22 \\
$\mathrm{H}(2 \mathrm{~B})$ & 5342 & 3061 & 7487 & 21 \\
$\mathrm{H}(2 \mathrm{C})$ & 4872 & 3938 & 7722 & 21 \\
$\mathrm{H}(5)$ & 5852 & 3749 & 8546 & 21 \\
$\mathrm{H}(6)$ & 5747 & 5441 & 1156 & 15 \\
$\mathrm{H}(7)$ & 7499 & 6725 & 826 & 16 \\
$\mathrm{H}(8)$ & 8341 & 7339 & 2929 & 17 \\
$\mathrm{H}(10)$ & 8416 & 6711 & 5442 & 16 \\
$\mathrm{H}(11)$ & 4235 & 3256 & 5311 & 15 \\
$\mathrm{H}(12)$ & 3595 & 3165 & 2700 & 16 \\
$\mathrm{H}(13)$ & 4360 & 3736 & 607 & 16 \\
\hline
\end{tabular}


c) Structural Data for $1 \cdot[\mathrm{F}]\left[\mathrm{C}_{6} \mathrm{~F}_{4} \mathrm{CN}\right]$

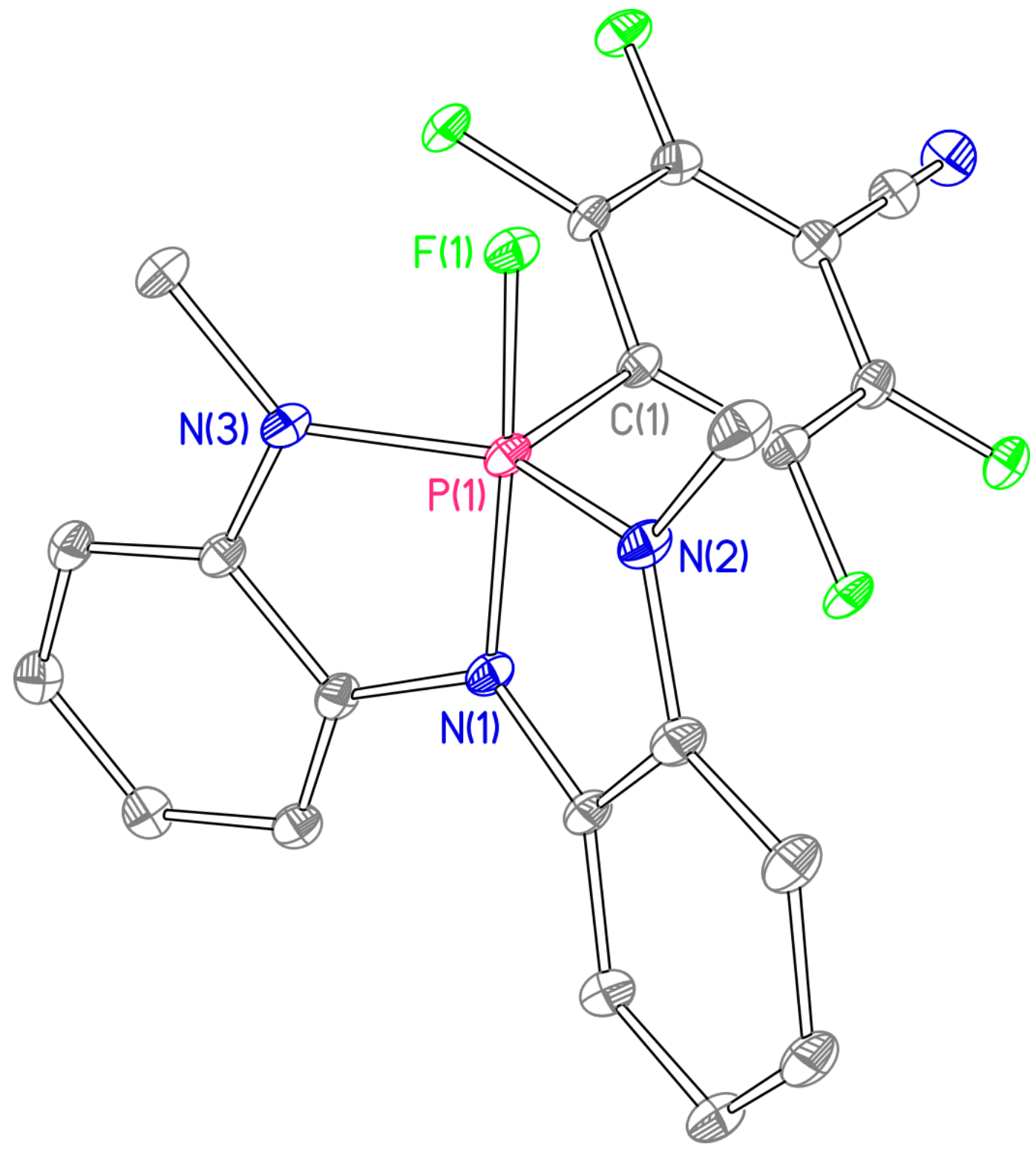

Figure S11. Thermal ellipsoid plot rendered at the $50 \%$ probability level for $\mathbf{1} \cdot[\mathrm{F}]\left[\mathrm{C}_{6} \mathrm{~F}_{4} \mathrm{CN}\right]$. Hydrogen atoms are omitted for clarity. 
Table S11. Crystal data and structure refinement for $\mathbf{1} \cdot[\mathrm{F}]\left[\mathrm{C}_{6} \mathrm{~F}_{4} \mathrm{CN}\right]$.

Identification code

Empirical formula

Formula weight

Temperature

Wavelength

Crystal system

Space group

Unit cell dimensions

Volume

Z

Density (calculated)

Absorption coefficient

$\mathrm{F}(000)$

Crystal size

Theta range for data collection

Index ranges

Reflections collected

Independent reflections

Completeness to theta $=14.357^{\circ}$

Absorption correction

Refinement method

Data / restraints / parameters

Goodness-of-fit on $\mathrm{F}^{2}$

Final R indices [I $>2$ sigma(I)]

$\mathrm{R}$ indices (all data)

Absolute structure parameter

Extinction coefficient

Largest diff. peak and hole
SL2-009

C21 H14 F5 N4 P

448.33

273(2) K

$0.41328 \AA$

Orthorhombic

Pna21

$\mathrm{a}=11.5328(5) \AA$

$\mathrm{a}=90^{\circ}$.

$\mathrm{b}=9.9328(5) \AA$

$\mathrm{b}=90^{\circ}$.

$\mathrm{c}=16.6188(8) \AA$

$\mathrm{g}=90^{\circ}$.
4

$1.564 \mathrm{Mg} / \mathrm{m}^{3}$

$0.064 \mathrm{~mm}^{-1}$

912

$0.250 \times 0.160 \times 0.150 \mathrm{~mm}^{3}$

1.389 to $17.548^{\circ}$.

$-16<=\mathrm{h}<=16,-14<=\mathrm{k}<=14,-23<=\mathrm{l}<=23$

55002

$6063[\mathrm{R}(\mathrm{int})=0.6030]$

$98.8 \%$

None

Full-matrix least-squares on $\mathrm{F}^{2}$

6063 / 1 / 282

1.088

$\mathrm{R} 1=0.0530, \mathrm{wR} 2=0.1414$

$\mathrm{R} 1=0.0581, \mathrm{wR} 2=0.1450$

$0.0(4)$

$\mathrm{n} / \mathrm{a}$

0.461 and -0.840 e. $\AA^{-3}$ 
Table S12. Atomic coordinates $\left(\times 10^{4}\right)$ and equivalent isotropic displacement parameters $\left(\AA^{2} \times 10^{3}\right)$ for 1•[F] $\left[\mathrm{C}_{6} \mathrm{~F}_{4} \mathrm{CN}\right] . \mathrm{U}(\mathrm{eq})$ is defined as one third of the trace of the orthogonalized $\mathrm{U}^{\mathrm{ij}}$ tensor.

\begin{tabular}{|c|c|c|c|c|}
\hline & $\mathrm{x}$ & $\mathrm{y}$ & $\mathrm{z}$ & $\mathrm{U}(\mathrm{eq})$ \\
\hline $\mathrm{P}(1)$ & $3260(1)$ & $5274(1)$ & $4903(1)$ & $10(1)$ \\
\hline$F(1)$ & $2171(2)$ & $5719(2)$ & $5474(1)$ & $13(1)$ \\
\hline $\mathrm{N}(3)$ & $2836(2)$ & $6407(3)$ & $4206(2)$ & $12(1)$ \\
\hline $\mathrm{N}(4)$ & $7357(3)$ & $8595(3)$ & $7575(2)$ & $21(1)$ \\
\hline $\mathrm{C}(1)$ & $2155(3)$ & $3192(3)$ & $5744(2)$ & $16(1)$ \\
\hline$N(2)$ & $3012(2)$ & $3657(2)$ & $5146(2)$ & $12(1)$ \\
\hline $\mathrm{C}(3)$ & $3699(3)$ & 2701(3) & $4741(2)$ & $13(1)$ \\
\hline$C(4)$ & $3655(3)$ & $1304(3)$ & $4817(2)$ & $15(1)$ \\
\hline$C(5)$ & $4438(3)$ & 531(3) & $4368(2)$ & $16(1)$ \\
\hline$C(6)$ & $5233(3)$ & $1132(3)$ & $3854(2)$ & $16(1)$ \\
\hline $\mathrm{C}(7)$ & $5265(3)$ & $2539(3)$ & $3771(2)$ & $14(1)$ \\
\hline$C(8)$ & $4484(3)$ & $3307(3)$ & $4209(2)$ & $12(1)$ \\
\hline $\mathrm{N}(1)$ & $4322(2)$ & $4710(2)$ & $4220(2)$ & $12(1)$ \\
\hline$C(9)$ & $3636(3)$ & $6579(3)$ & $3575(2)$ & $12(1)$ \\
\hline$C(10)$ & $3643(3)$ & $7610(3)$ & $3006(2)$ & $14(1)$ \\
\hline$C(11)$ & $4526(3)$ & $7624(3)$ & $2428(2)$ & $15(1)$ \\
\hline$C(12)$ & $5369(3)$ & $6614(3)$ & $2421(2)$ & $14(1)$ \\
\hline$C(13)$ & $5368(3)$ & $5587(3)$ & 2998(2) & $13(1)$ \\
\hline$C(14)$ & $4506(3)$ & $5591(3)$ & $3576(2)$ & $12(1)$ \\
\hline$C(15)$ & $4279(2)$ & $6104(3)$ & $5610(2)$ & $11(1)$ \\
\hline$C(16)$ & $5245(3)$ & $5443(3)$ & $5915(2)$ & $12(1)$ \\
\hline$F(2)$ & $5470(2)$ & $4164(2)$ & $5709(1)$ & $14(1)$ \\
\hline$C(17)$ & $6012(3)$ & $6046(3)$ & $6448(2)$ & $13(1)$ \\
\hline$F(3)$ & $6916(2)$ & $5353(2)$ & $6733(1)$ & $16(1)$ \\
\hline$C(18)$ & $5855(3)$ & $7389(3)$ & $6679(2)$ & $14(1)$ \\
\hline$C(19)$ & $4895(3)$ & $8073(3)$ & $6372(2)$ & $14(1)$ \\
\hline$F(4)$ & $4720(2)$ & $9357(2)$ & $6579(1)$ & $17(1)$ \\
\hline$C(20)$ & $4127(3)$ & $7429(3)$ & $5856(2)$ & $12(1)$ \\
\hline$F(5)$ & $3225(2)$ & $8165(2)$ & $5590(1)$ & $14(1)$ \\
\hline$C(21)$ & $6679(3)$ & $8044(3)$ & $7187(2)$ & $16(1)$ \\
\hline $\mathrm{C}(2)$ & $1819(3)$ & $7292(3)$ & $4213(2)$ & $16(1)$ \\
\hline
\end{tabular}


Table S13. Bond lengths $[\AA]$ and angles $\left[^{\circ}\right]$ for $\mathbf{1} \cdot[\mathrm{F}]\left[\mathrm{C}_{6} \mathrm{~F}_{4} \mathrm{CN}\right]$.

\begin{tabular}{|c|c|}
\hline$P(1)-F(1)$ & $1.634(2)$ \\
\hline$P(1)-N(2)$ & $1.681(3)$ \\
\hline$P(1)-N(3)$ & $1.687(3)$ \\
\hline$P(1)-N(1)$ & $1.761(3)$ \\
\hline$P(1)-C(15)$ & $1.855(3)$ \\
\hline$N(3)-C(9)$ & $1.407(4)$ \\
\hline $\mathrm{N}(3)-\mathrm{C}(2)$ & $1.466(4)$ \\
\hline$N(4)-C(21)$ & $1.153(5)$ \\
\hline $\mathrm{C}(1)-\mathrm{N}(2)$ & $1.475(4)$ \\
\hline $\mathrm{C}(1)-\mathrm{H}(1 \mathrm{~A})$ & 0.9600 \\
\hline $\mathrm{C}(1)-\mathrm{H}(1 \mathrm{~B})$ & 0.9600 \\
\hline $\mathrm{C}(1)-\mathrm{H}(1 \mathrm{C})$ & 0.9600 \\
\hline$N(2)-C(3)$ & $1.408(4)$ \\
\hline$C(3)-C(4)$ & $1.394(4)$ \\
\hline$C(3)-C(8)$ & $1.401(4)$ \\
\hline$C(4)-C(5)$ & $1.401(4)$ \\
\hline $\mathrm{C}(4)-\mathrm{H}(4)$ & 0.9300 \\
\hline$C(5)-C(6)$ & $1.387(5)$ \\
\hline $\mathrm{C}(5)-\mathrm{H}(5)$ & 0.9300 \\
\hline$C(6)-C(7)$ & $1.405(4)$ \\
\hline $\mathrm{C}(6)-\mathrm{H}(6)$ & 0.9300 \\
\hline$C(7)-C(8)$ & $1.387(4)$ \\
\hline $\mathrm{C}(7)-\mathrm{H}(7)$ & 0.9300 \\
\hline $\mathrm{C}(8)-\mathrm{N}(1)$ & $1.406(4)$ \\
\hline$N(1)-C(14)$ & $1.400(4)$ \\
\hline $\mathrm{C}(9)-\mathrm{C}(10)$ & $1.393(4)$ \\
\hline$C(9)-C(14)$ & $1.404(4)$ \\
\hline$C(10)-C(11)$ & $1.401(5)$ \\
\hline $\mathrm{C}(10)-\mathrm{H}(10)$ & 0.9300 \\
\hline $\mathrm{C}(11)-\mathrm{C}(12)$ & $1.397(5)$ \\
\hline $\mathrm{C}(11)-\mathrm{H}(11)$ & 0.9300 \\
\hline$C(12)-C(13)$ & $1.400(4)$ \\
\hline $\mathrm{C}(12)-\mathrm{H}(12)$ & 0.9300 \\
\hline$C(13)-C(14)$ & $1.383(4)$ \\
\hline $\mathrm{C}(13)-\mathrm{H}(13)$ & 0.9300 \\
\hline
\end{tabular}




\begin{tabular}{|c|c|}
\hline$C(15)-C(16)$ & $1.389(4)$ \\
\hline$C(15)-C(20)$ & $1.390(4)$ \\
\hline$C(16)-F(2)$ & $1.341(3)$ \\
\hline$C(16)-C(17)$ & $1.387(4)$ \\
\hline$C(17)-F(3)$ & $1.337(3)$ \\
\hline$C(17)-C(18)$ & $1.400(4)$ \\
\hline$C(18)-C(19)$ & $1.395(4)$ \\
\hline$C(18)-C(21)$ & $1.428(4)$ \\
\hline$C(19)-F(4)$ & $1.337(3)$ \\
\hline$C(19)-C(20)$ & $1.389(4)$ \\
\hline$C(20)-F(5)$ & $1.346(3)$ \\
\hline $\mathrm{C}(2)-\mathrm{H}(2 \mathrm{~A})$ & 0.9600 \\
\hline $\mathrm{C}(2)-\mathrm{H}(2 \mathrm{~B})$ & 0.9600 \\
\hline $\mathrm{C}(2)-\mathrm{H}(2 \mathrm{C})$ & 0.9600 \\
\hline$F(1)-P(1)-N(2)$ & $89.34(12)$ \\
\hline$F(1)-P(1)-N(3)$ & $89.77(12)$ \\
\hline$N(2)-P(1)-N(3)$ & $138.88(14)$ \\
\hline$F(1)-P(1)-N(1)$ & $173.84(12)$ \\
\hline$N(2)-P(1)-N(1)$ & $88.23(13)$ \\
\hline$N(3)-P(1)-N(1)$ & $88.34(13)$ \\
\hline$F(1)-P(1)-C(15)$ & $89.93(12)$ \\
\hline$N(2)-P(1)-C(15)$ & $112.34(14)$ \\
\hline$N(3)-P(1)-C(15)$ & $108.77(13)$ \\
\hline$N(1)-P(1)-C(15)$ & $96.23(13)$ \\
\hline $\mathrm{C}(9)-\mathrm{N}(3)-\mathrm{C}(2)$ & $117.2(3)$ \\
\hline$C(9)-N(3)-P(1)$ & $113.8(2)$ \\
\hline$C(2)-N(3)-P(1)$ & $128.8(2)$ \\
\hline$N(2)-C(1)-H(1 A)$ & 109.5 \\
\hline $\mathrm{N}(2)-\mathrm{C}(1)-\mathrm{H}(1 \mathrm{~B})$ & 109.5 \\
\hline$H(1 A)-C(1)-H(1 B)$ & 109.5 \\
\hline $\mathrm{N}(2)-\mathrm{C}(1)-\mathrm{H}(1 \mathrm{C})$ & 109.5 \\
\hline$H(1 A)-C(1)-H(1 C)$ & 109.5 \\
\hline $\mathrm{H}(1 \mathrm{~B})-\mathrm{C}(1)-\mathrm{H}(1 \mathrm{C})$ & 109.5 \\
\hline$C(3)-N(2)-C(1)$ & $119.2(2)$ \\
\hline$C(3)-N(2)-P(1)$ & $115.7(2)$ \\
\hline$C(1)-N(2)-P(1)$ & $125.1(2)$ \\
\hline
\end{tabular}




\begin{tabular}{|c|c|}
\hline$C(4)-C(3)-C(8)$ & $120.6(3)$ \\
\hline $\mathrm{C}(4)-\mathrm{C}(3)-\mathrm{N}(2)$ & $127.4(3)$ \\
\hline $\mathrm{C}(8)-\mathrm{C}(3)-\mathrm{N}(2)$ & $112.0(2)$ \\
\hline$C(3)-C(4)-C(5)$ & $118.3(3)$ \\
\hline $\mathrm{C}(3)-\mathrm{C}(4)-\mathrm{H}(4)$ & 120.9 \\
\hline $\mathrm{C}(5)-\mathrm{C}(4)-\mathrm{H}(4)$ & 120.9 \\
\hline$C(6)-C(5)-C(4)$ & $121.2(3)$ \\
\hline $\mathrm{C}(6)-\mathrm{C}(5)-\mathrm{H}(5)$ & 119.4 \\
\hline $\mathrm{C}(4)-\mathrm{C}(5)-\mathrm{H}(5)$ & 119.4 \\
\hline$C(5)-C(6)-C(7)$ & $120.4(3)$ \\
\hline$C(5)-C(6)-H(6)$ & 119.8 \\
\hline $\mathrm{C}(7)-\mathrm{C}(6)-\mathrm{H}(6)$ & 119.8 \\
\hline$C(8)-C(7)-C(6)$ & $118.6(3)$ \\
\hline $\mathrm{C}(8)-\mathrm{C}(7)-\mathrm{H}(7)$ & 120.7 \\
\hline $\mathrm{C}(6)-\mathrm{C}(7)-\mathrm{H}(7)$ & 120.7 \\
\hline$C(7)-C(8)-C(3)$ & $120.9(3)$ \\
\hline $\mathrm{C}(7)-\mathrm{C}(8)-\mathrm{N}(1)$ & $129.7(3)$ \\
\hline $\mathrm{C}(3)-\mathrm{C}(8)-\mathrm{N}(1)$ & $109.4(3)$ \\
\hline$C(14)-N(1)-C(8)$ & $126.1(3)$ \\
\hline$C(14)-N(1)-P(1)$ & $113.6(2)$ \\
\hline$C(8)-N(1)-P(1)$ & $114.6(2)$ \\
\hline$C(10)-C(9)-C(14)$ & $120.7(3)$ \\
\hline$C(10)-C(9)-N(3)$ & $126.8(3)$ \\
\hline$C(14)-C(9)-N(3)$ & $112.4(3)$ \\
\hline$C(9)-C(10)-C(11)$ & $118.5(3)$ \\
\hline$C(9)-C(10)-H(10)$ & 120.7 \\
\hline $\mathrm{C}(11)-\mathrm{C}(10)-\mathrm{H}(10)$ & 120.7 \\
\hline$C(12)-C(11)-C(10)$ & $120.3(3)$ \\
\hline $\mathrm{C}(12)-\mathrm{C}(11)-\mathrm{H}(11)$ & 119.9 \\
\hline $\mathrm{C}(10)-\mathrm{C}(11)-\mathrm{H}(11)$ & 119.9 \\
\hline$C(11)-C(12)-C(13)$ & $121.1(3)$ \\
\hline $\mathrm{C}(11)-\mathrm{C}(12)-\mathrm{H}(12)$ & 119.4 \\
\hline $\mathrm{C}(13)-\mathrm{C}(12)-\mathrm{H}(12)$ & 119.4 \\
\hline$C(14)-C(13)-C(12)$ & $118.3(3)$ \\
\hline $\mathrm{C}(14)-\mathrm{C}(13)-\mathrm{H}(13)$ & 120.8 \\
\hline $\mathrm{C}(12)-\mathrm{C}(13)-\mathrm{H}(13)$ & 120.8 \\
\hline$C(13)-C(14)-N(1)$ & $129.7(3)$ \\
\hline
\end{tabular}




\begin{tabular}{ll}
$\mathrm{C}(13)-\mathrm{C}(14)-\mathrm{C}(9)$ & $121.0(3)$ \\
$\mathrm{N}(1)-\mathrm{C}(14)-\mathrm{C}(9)$ & $109.3(3)$ \\
$\mathrm{C}(16)-\mathrm{C}(15)-\mathrm{C}(20)$ & $116.2(3)$ \\
$\mathrm{C}(16)-\mathrm{C}(15)-\mathrm{P}(1)$ & $122.0(2)$ \\
$\mathrm{C}(20)-\mathrm{C}(15)-\mathrm{P}(1)$ & $121.8(2)$ \\
$\mathrm{F}(2)-\mathrm{C}(16)-\mathrm{C}(17)$ & $116.7(3)$ \\
$\mathrm{F}(2)-\mathrm{C}(16)-\mathrm{C}(15)$ & $120.6(3)$ \\
$\mathrm{C}(17)-\mathrm{C}(16)-\mathrm{C}(15)$ & $122.7(3)$ \\
$\mathrm{F}(3)-\mathrm{C}(17)-\mathrm{C}(16)$ & $120.1(3)$ \\
$\mathrm{F}(3)-\mathrm{C}(17)-\mathrm{C}(18)$ & $119.6(3)$ \\
$\mathrm{C}(16)-\mathrm{C}(17)-\mathrm{C}(18)$ & $120.3(3)$ \\
$\mathrm{C}(19)-\mathrm{C}(18)-\mathrm{C}(17)$ & $117.8(3)$ \\
$\mathrm{C}(19)-\mathrm{C}(18)-\mathrm{C}(21)$ & $121.5(3)$ \\
$\mathrm{C}(17)-\mathrm{C}(18)-\mathrm{C}(21)$ & $120.7(3)$ \\
$\mathrm{F}(4)-\mathrm{C}(19)-\mathrm{C}(20)$ & $120.1(3)$ \\
$\mathrm{F}(4)-\mathrm{C}(19)-\mathrm{C}(18)$ & $119.4(3)$ \\
$\mathrm{C}(20)-\mathrm{C}(19)-\mathrm{C}(18)$ & $120.5(3)$ \\
$\mathrm{F}(5)-\mathrm{C}(20)-\mathrm{C}(19)$ & $116.5(2)$ \\
$\mathrm{F}(5)-\mathrm{C}(20)-\mathrm{C}(15)$ & $121.1(3)$ \\
$\mathrm{C}(19)-\mathrm{C}(20)-\mathrm{C}(15)$ & $122.5(3)$ \\
$\mathrm{N}(4)-\mathrm{C}(21)-\mathrm{C}(18)$ & $177.8(4)$ \\
$\mathrm{N}(3)-\mathrm{C}(2)-\mathrm{H}(2 \mathrm{~A})$ & 109.5 \\
$\mathrm{~N}(3)-\mathrm{C}(2)-\mathrm{H}(2 \mathrm{~B})$ & 109.5 \\
$\mathrm{H}(2 \mathrm{~A})-\mathrm{C}(2)-\mathrm{H}(2 \mathrm{~B})$ & 109.5 \\
$\mathrm{~N}(3)-\mathrm{C}(2)-\mathrm{H}(2 \mathrm{C})$ & 109.5 \\
$\mathrm{H}(2 \mathrm{~A})-\mathrm{C}(2)-\mathrm{H}(2 \mathrm{C})$ & 109.5 \\
$\mathrm{H}(2 \mathrm{~B})-\mathrm{C}(2)-\mathrm{H}(2 \mathrm{C})$ & 109.5 \\
& \\
\hline
\end{tabular}

Symmetry transformations used to generate equivalent atoms: 
Table S14. Anisotropic displacement parameters $\left(\AA^{2} \times 10^{3}\right)$ for $\mathbf{1} \cdot[\mathrm{F}]\left[\mathrm{C}_{6} \mathrm{~F}_{4} \mathrm{CN}\right]$. The anisotropic displacement factor exponent takes the form: $-2 p^{2}\left[h^{2} a^{* 2} U^{11}+\ldots+2 h k a * b^{*} U^{12}\right]$

\begin{tabular}{|c|c|c|c|c|c|c|}
\hline & $\mathrm{U}^{11}$ & $\mathrm{U}^{22}$ & $\mathrm{U}^{33}$ & $\mathrm{U}^{23}$ & $\mathrm{U}^{13}$ & $\mathrm{U}^{12}$ \\
\hline$P(1)$ & $10(1)$ & $6(1)$ & $14(1)$ & $0(1)$ & $0(1)$ & $0(1)$ \\
\hline $\mathrm{F}(1)$ & $12(1)$ & $10(1)$ & $17(1)$ & $0(1)$ & $2(1)$ & $1(1)$ \\
\hline $\mathrm{N}(3)$ & $11(1)$ & $10(1)$ & $16(1)$ & $0(1)$ & $2(1)$ & $2(1)$ \\
\hline $\mathrm{N}(4)$ & $19(1)$ & $20(1)$ & $24(1)$ & $-1(1)$ & $-2(1)$ & $-3(1)$ \\
\hline $\mathrm{C}(1)$ & $14(1)$ & $10(1)$ & $23(2)$ & $3(1)$ & $4(1)$ & $-1(1)$ \\
\hline $\mathrm{N}(2)$ & $13(1)$ & $6(1)$ & $17(1)$ & $1(1)$ & $2(1)$ & $-1(1)$ \\
\hline$C(3)$ & $13(1)$ & $10(1)$ & $17(1)$ & $-2(1)$ & $-1(1)$ & $0(1)$ \\
\hline$C(4)$ & $16(1)$ & $8(1)$ & $22(2)$ & $1(1)$ & $-1(1)$ & $-1(1)$ \\
\hline$C(5)$ & $18(2)$ & $7(1)$ & $22(1)$ & $1(1)$ & $-1(1)$ & $-1(1)$ \\
\hline$C(6)$ & $16(1)$ & $10(1)$ & $21(1)$ & $0(1)$ & $1(1)$ & $2(1)$ \\
\hline$C(7)$ & $14(1)$ & $12(1)$ & $18(1)$ & $-2(1)$ & $1(1)$ & $1(1)$ \\
\hline$C(8)$ & $12(1)$ & $7(1)$ & $15(1)$ & $0(1)$ & $-1(1)$ & $0(1)$ \\
\hline $\mathrm{N}(1)$ & $12(1)$ & $8(1)$ & $15(1)$ & $1(1)$ & $1(1)$ & $1(1)$ \\
\hline$C(9)$ & $13(1)$ & $8(1)$ & $15(1)$ & $0(1)$ & $0(1)$ & $0(1)$ \\
\hline$C(10)$ & $14(1)$ & $11(1)$ & $17(1)$ & $2(1)$ & $-2(1)$ & $1(1)$ \\
\hline$C(11)$ & $16(1)$ & $13(1)$ & $17(1)$ & $2(1)$ & $-1(1)$ & $-3(1)$ \\
\hline$C(12)$ & $15(1)$ & $12(1)$ & $16(1)$ & $1(1)$ & $1(1)$ & $-5(1)$ \\
\hline$C(13)$ & $14(1)$ & $9(1)$ & $17(1)$ & $-1(1)$ & $0(1)$ & $-1(1)$ \\
\hline$C(14)$ & $13(1)$ & $7(1)$ & $15(1)$ & $1(1)$ & $-1(1)$ & $-1(1)$ \\
\hline$C(15)$ & $10(1)$ & $8(1)$ & $15(1)$ & $1(1)$ & $0(1)$ & $0(1)$ \\
\hline$C(16)$ & $12(1)$ & $9(1)$ & $16(1)$ & $0(1)$ & $1(1)$ & $0(1)$ \\
\hline$F(2)$ & $15(1)$ & $7(1)$ & $20(1)$ & $0(1)$ & $-1(1)$ & $3(1)$ \\
\hline $\mathrm{C}(17)$ & $12(1)$ & $10(1)$ & $17(1)$ & $2(1)$ & $-2(1)$ & $-1(1)$ \\
\hline $\mathrm{F}(3)$ & $13(1)$ & $15(1)$ & $20(1)$ & $2(1)$ & $-3(1)$ & $2(1)$ \\
\hline$C(18)$ & $13(1)$ & $12(1)$ & $16(1)$ & $-1(1)$ & $-1(1)$ & $-2(1)$ \\
\hline $\mathrm{C}(19)$ & $16(1)$ & $8(1)$ & $17(1)$ & $-2(1)$ & $1(1)$ & $-2(1)$ \\
\hline$F(4)$ & $18(1)$ & $9(1)$ & $24(1)$ & $-4(1)$ & $-2(1)$ & $0(1)$ \\
\hline$C(20)$ & $12(1)$ & $8(1)$ & $16(1)$ & $1(1)$ & $-1(1)$ & $2(1)$ \\
\hline$F(5)$ & $13(1)$ & $10(1)$ & $20(1)$ & $0(1)$ & $-3(1)$ & $3(1)$ \\
\hline$C(21)$ & $15(2)$ & $16(1)$ & $19(1)$ & $0(1)$ & $0(1)$ & $-2(1)$ \\
\hline$C(2)$ & $15(1)$ & $12(1)$ & $20(1)$ & $1(1)$ & $0(1)$ & $5(1)$ \\
\hline
\end{tabular}


Table S15. Hydrogen coordinates $\left(\times 10^{4}\right)$ and isotropic displacement parameters $\left(\AA^{2} \times 10^{3}\right)$ for 1•[F][C $\left.\mathrm{C}_{6} \mathrm{~F}_{4} \mathrm{CN}\right]$.

\begin{tabular}{|c|c|c|c|c|}
\hline & $\mathrm{x}$ & $\mathrm{y}$ & $\mathrm{z}$ & $\mathrm{U}(\mathrm{eq})$ \\
\hline $\mathrm{H}(1 \mathrm{~A})$ & 1420 & 3057 & 5485 & 24 \\
\hline $\mathrm{H}(1 \mathrm{~B})$ & 2075 & 3857 & 6159 & 24 \\
\hline $\mathrm{H}(1 \mathrm{C})$ & 2413 & 2359 & 5976 & 24 \\
\hline $\mathrm{H}(4)$ & 3120 & 896 & 5158 & 18 \\
\hline $\mathrm{H}(5)$ & 4424 & -402 & 4415 & 19 \\
\hline $\mathrm{H}(6)$ & 5747 & 600 & 3564 & 19 \\
\hline $\mathrm{H}(7)$ & 5798 & 2946 & 3429 & 17 \\
\hline $\mathrm{H}(10)$ & 3074 & 8273 & 3011 & 17 \\
\hline $\mathrm{H}(11)$ & 4552 & 8310 & 2047 & 18 \\
\hline $\mathrm{H}(12)$ & 5940 & 6623 & 2026 & 17 \\
\hline $\mathrm{H}(13)$ & 5933 & 4919 & 2992 & 16 \\
\hline $\mathrm{H}(2 \mathrm{~A})$ & 2064 & 8207 & 4291 & 23 \\
\hline $\mathrm{H}(2 \mathrm{~B})$ & 1310 & 7032 & 4643 & 23 \\
\hline $\mathrm{H}(2 \mathrm{C})$ & 1417 & 7218 & 3709 & 23 \\
\hline
\end{tabular}




\section{d) Structural Data for $1 \cdot[\mathrm{H}]\left[\mathrm{C}_{5} \mathrm{~F}_{4} \mathrm{~N}\right]$}

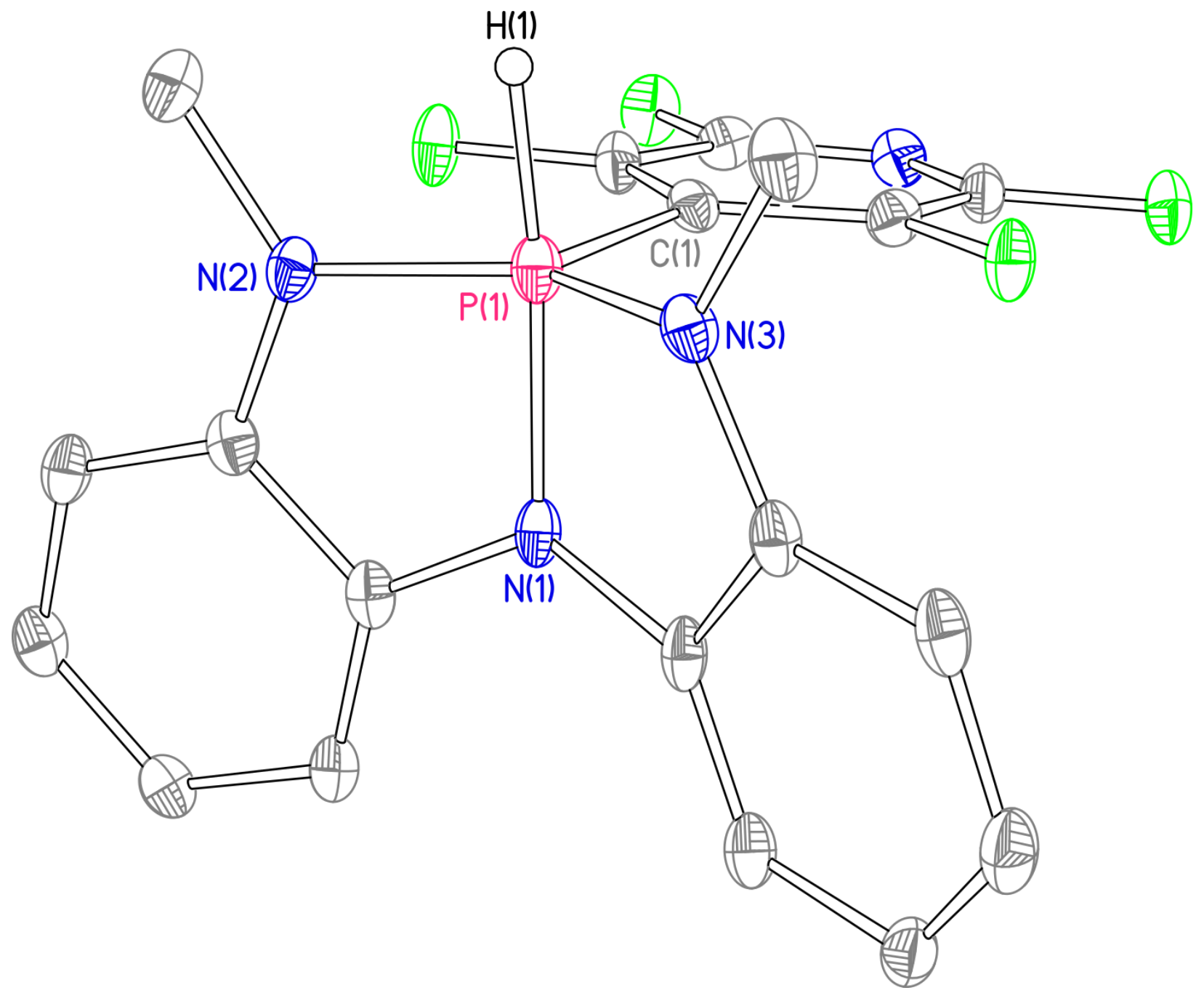

Figure S12. Thermal ellipsoid plot rendered at the $50 \%$ probability level for $1 \bullet[H]\left[\mathrm{C}_{5} \mathrm{~F}_{4} \mathrm{~N}\right]$. Hydrogen atoms except H(1) are omitted for clarity. 
Table S16. Crystal data and structure refinement for $\mathbf{1} \cdot[\mathrm{H}]\left[\mathrm{C}_{5} \mathrm{~F}_{4} \mathrm{~N}\right]$.

Identification code

Empirical formula

Formula weight

Temperature

Wavelength

Crystal system

Space group

Unit cell dimensions

Volume

Z

Density (calculated)

Absorption coefficient

$\mathrm{F}(000)$

Crystal size

Theta range for data collection

Index ranges

Reflections collected

Independent reflections

Completeness to theta $=14.357^{\circ}$

Absorption correction

Refinement method

Data / restraints / parameters

Goodness-of-fit on $\mathrm{F}^{2}$

Final R indices [I $>2$ sigma(I)]

$\mathrm{R}$ indices (all data)

Extinction coefficient

Largest diff. peak and hole
SL2-044

C19 H15 F4 N4 P

406.32

273(2) K

$0.41328 \AA$

Monoclinic

$\mathrm{P} 21 / \mathrm{c}$

$\mathrm{a}=11.7841(10) \AA$

$\mathrm{a}=90^{\circ}$.

$\mathrm{b}=37.014(3) \AA$

$\mathrm{b}=109.033(2)^{\circ}$.

$c=8.2426(6) \AA$

$\mathrm{g}=90^{\circ}$.

3398.7(5) $\AA^{3}$

8

$1.588 \mathrm{Mg} / \mathrm{m}^{3}$

$0.066 \mathrm{~mm}^{-1}$

1664

$0.300 \times 0.050 \times 0.050 \mathrm{~mm}^{3}$

1.063 to $14.589^{\circ}$.

$-14<=\mathrm{h}<=14,-45<=\mathrm{k}<=45,-9<=\mathrm{l}<=9$

69045

$6229[\mathrm{R}(\mathrm{int})=0.1209]$

$96.7 \%$

None

Full-matrix least-squares on $\mathrm{F}^{2}$

6229 / 49 / 515

0.926

$\mathrm{R} 1=0.0431, \mathrm{wR} 2=0.1024$

$\mathrm{R} 1=0.0593, \mathrm{wR} 2=0.1065$

$\mathrm{n} / \mathrm{a}$

0.501 and -0.510 e. $\AA^{-3}$ 
Table S17. Atomic coordinates $\left(\times 10^{4}\right)$ and equivalent isotropic displacement parameters $\left(\AA^{2} \times 10^{3}\right)$ for $\mathbf{1 \cdot}[\mathrm{H}]\left[\mathrm{C}_{5} \mathrm{~F}_{4} \mathrm{~N}\right] . \mathrm{U}(\mathrm{eq})$ is defined as one third of the trace of the orthogonalized $\mathrm{U}^{\mathrm{ij}}$ tensor.

\begin{tabular}{|c|c|c|c|c|}
\hline & $\mathrm{x}$ & $\mathrm{y}$ & $\mathrm{z}$ & $\mathrm{U}(\mathrm{eq})$ \\
\hline $\mathrm{P}(1)$ & $7025(1)$ & $6454(1)$ & $3433(1)$ & $14(1)$ \\
\hline$C(11)$ & $7786(2)$ & $5848(1)$ & $2108(3)$ & $19(1)$ \\
\hline$N(12)$ & $6882(2)$ & $6022(1)$ & $2692(2)$ & $15(1)$ \\
\hline$C(13)$ & $5999(2)$ & $5812(1)$ & $3063(3)$ & $15(1)$ \\
\hline$C(14)$ & $5790(2)$ & $5446(1)$ & $2798(3)$ & $18(1)$ \\
\hline$C(15)$ & $4845(2)$ & $5291(1)$ & $3212(3)$ & $18(1)$ \\
\hline$C(16)$ & $4109(2)$ & $5500(1)$ & $3843(3)$ & $17(1)$ \\
\hline$C(17)$ & $4314(2)$ & $5871(1)$ & $4113(3)$ & $16(1)$ \\
\hline$C(18)$ & $5270(2)$ & $6025(1)$ & $3732(3)$ & $15(1)$ \\
\hline $\mathrm{N}(11)$ & $5672(2)$ & $6383(1)$ & $3901(2)$ & $14(1)$ \\
\hline $\mathrm{C}(114)$ & $5014(2)$ & $6697(1)$ & $3910(3)$ & $15(1)$ \\
\hline C(113) & $4045(2)$ & $6757(1)$ & $4471(3)$ & $16(1)$ \\
\hline $\mathrm{C}(112)$ & $3532(2)$ & $7103(1)$ & $4288(3)$ & $18(1)$ \\
\hline $\mathrm{C}(111)$ & $3992(2)$ & 7381(1) & $3560(3)$ & $21(1)$ \\
\hline $\mathrm{C}(110)$ & $4986(2)$ & $7324(1)$ & $3022(3)$ & $18(1)$ \\
\hline$C(19)$ & $5496(2)$ & $6982(1)$ & $3209(3)$ & $15(1)$ \\
\hline$N(13)$ & $6492(2)$ & $6872(1)$ & $2762(2)$ & $15(1)$ \\
\hline$C(12)$ & $7149(2)$ & $7142(1)$ & $2129(3)$ & $19(1)$ \\
\hline$N(14)$ & $9277(2)$ & $6457(1)$ & $9339(2)$ & $18(1)$ \\
\hline$F(11)$ & $8502(1)$ & $5830(1)$ & $5864(2)$ & $21(1)$ \\
\hline$F(12)$ & $9662(1)$ & $5861(1)$ & $9201(2)$ & $22(1)$ \\
\hline$F(13)$ & $8905(1)$ & $7052(1)$ & $9461(2)$ & $21(1)$ \\
\hline$F(14)$ & 7703(1) & $7088(1)$ & $6126(2)$ & $19(1)$ \\
\hline$C(115)$ & $8002(2)$ & $6458(1)$ & $5753(3)$ & $14(1)$ \\
\hline$C(116)$ & $8558(2)$ & $6153(1)$ & $6653(3)$ & $17(1)$ \\
\hline $\mathrm{C}(117)$ & $9157(2)$ & $6167(1)$ & $8383(3)$ & $16(1)$ \\
\hline C(118) & $8782(2)$ & $6748(1)$ & $8513(3)$ & $17(1)$ \\
\hline $\mathrm{C}(119)$ & $8163(2)$ & $6765(1)$ & $6778(3)$ & $15(1)$ \\
\hline$C(21)$ & $2242(2)$ & $6585(1)$ & $10609(3)$ & 18(1) \\
\hline$C(22)$ & $2203(2)$ & $5308(1)$ & $9244(3)$ & 19(1) \\
\hline$C(23)$ & $1439(2)$ & $6661(1)$ & $7470(3)$ & $16(1)$ \\
\hline \multirow[t]{2}{*}{$C(24)$} & $1112(2)$ & $7018(1)$ & $7492(3)$ & $17(1)$ \\
\hline & \multicolumn{4}{|c|}{ S47 } \\
\hline
\end{tabular}




\begin{tabular}{|c|c|c|c|c|}
\hline$C(25)$ & $580(2)$ & $7195(1)$ & $5925(3)$ & $19(1)$ \\
\hline$C(26)$ & $382(2)$ & $7015(1)$ & $4395(3)$ & $18(1)$ \\
\hline $\mathrm{C}(27)$ & $740(2)$ & $6654(1)$ & $4364(3)$ & $17(1)$ \\
\hline$C(28)$ & $1285(2)$ & $6479(1)$ & $5909(3)$ & $16(1)$ \\
\hline$C(29)$ & $1640(2)$ & $5509(1)$ & $6234(3)$ & $16(1)$ \\
\hline$C(210)$ & $1448(2)$ & $5169(1)$ & $5509(3)$ & $17(1)$ \\
\hline$C(211)$ & $1037(2)$ & $5136(1)$ & $3725(3)$ & $18(1)$ \\
\hline$C(212)$ & $825(2)$ & $5443(1)$ & $2703(3)$ & $18(1)$ \\
\hline$C(213)$ & $1039(2)$ & $5789(1)$ & $3429(3)$ & $17(1)$ \\
\hline$C(214)$ & $1449(2)$ & $5822(1)$ & $5196(3)$ & $15(1)$ \\
\hline$C(215)$ & $4037(2)$ & $6090(1)$ & $8438(3)$ & $15(1)$ \\
\hline$C(216)$ & $4586(2)$ & $6426(1)$ & $8520(3)$ & $15(1)$ \\
\hline$C(217)$ & $5718(2)$ & $6458(1)$ & $8414(3)$ & $16(1)$ \\
\hline$C(218)$ & $5879(2)$ & $5864(1)$ & $8143(3)$ & $17(1)$ \\
\hline$C(219)$ & $4767(2)$ & $5804(1)$ & $8279(3)$ & $17(1)$ \\
\hline $\mathrm{P}(2)$ & $2463(1)$ & $6030(1)$ & $8474(1)$ & $15(1)$ \\
\hline $\mathrm{N}(21)$ & $1738(2)$ & $6128(1)$ & $6249(2)$ & $15(1)$ \\
\hline $\mathrm{N}(22)$ & $1947(2)$ & $6434(1)$ & $8884(2)$ & $15(1)$ \\
\hline$N(23)$ & $2030(2)$ & $5597(1)$ & $7981(2)$ & $16(1)$ \\
\hline $\mathrm{N}(24)$ & $6379(2)$ & $6185(1)$ & $8214(2)$ & $17(1)$ \\
\hline $\mathrm{F}(21)$ & $4020(1)$ & $6734(1)$ & $8684(2)$ & $21(1)$ \\
\hline$F(22)$ & $6214(1)$ & $6788(1)$ & $8493(2)$ & $20(1)$ \\
\hline $\mathrm{F}(23)$ & $6542(1)$ & $5583(1)$ & $7934(2)$ & $22(1)$ \\
\hline$F(24)$ & $4388(1)$ & $5457(1)$ & $8221(2)$ & $20(1)$ \\
\hline
\end{tabular}


Table S18. Bond lengths $[\AA ̊]$ and angles $\left[^{\circ}\right]$ for $\mathbf{1} \cdot[\mathrm{H}]\left[\mathrm{C}_{5} \mathrm{~F}_{4} \mathrm{~N}\right]$.

\begin{tabular}{|c|c|}
\hline$P(1)-N(13)$ & $1.695(2)$ \\
\hline$P(1)-N(12)$ & $1.698(2)$ \\
\hline$P(1)-N(11)$ & $1.778(2)$ \\
\hline$P(1)-C(115)$ & $1.884(2)$ \\
\hline$P(1)-H(1)$ & $1.347(19)$ \\
\hline $\mathrm{C}(11)-\mathrm{N}(12)$ & $1.456(3)$ \\
\hline$C(11)-H(11 A)$ & 0.9600 \\
\hline $\mathrm{C}(11)-\mathrm{H}(11 \mathrm{~B})$ & 0.9600 \\
\hline $\mathrm{C}(11)-\mathrm{H}(11 \mathrm{C})$ & 0.9600 \\
\hline $\mathrm{N}(12)-\mathrm{C}(13)$ & $1.411(3)$ \\
\hline $\mathrm{C}(13)-\mathrm{C}(14)$ & $1.380(3)$ \\
\hline $\mathrm{C}(13)-\mathrm{C}(18)$ & $1.403(3)$ \\
\hline$C(14)-C(15)$ & $1.391(3)$ \\
\hline $\mathrm{C}(14)-\mathrm{H}(14)$ & 0.9300 \\
\hline$C(15)-C(16)$ & $1.384(3)$ \\
\hline $\mathrm{C}(15)-\mathrm{H}(15)$ & 0.9300 \\
\hline $\mathrm{C}(16)-\mathrm{C}(17)$ & $1.396(3)$ \\
\hline $\mathrm{C}(16)-\mathrm{H}(16)$ & 0.9300 \\
\hline $\mathrm{C}(17)-\mathrm{C}(18)$ & $1.387(3)$ \\
\hline $\mathrm{C}(17)-\mathrm{H}(17)$ & 0.9300 \\
\hline $\mathrm{C}(18)-\mathrm{N}(11)$ & $1.398(3)$ \\
\hline $\mathrm{N}(11)-\mathrm{C}(114)$ & $1.401(3)$ \\
\hline$C(114)-C(113)$ & $1.383(3)$ \\
\hline$C(114)-C(19)$ & $1.407(3)$ \\
\hline$C(113)-C(112)$ & $1.403(3)$ \\
\hline $\mathrm{C}(113)-\mathrm{H}(113)$ & 0.9300 \\
\hline$C(112)-C(111)$ & $1.389(3)$ \\
\hline $\mathrm{C}(112)-\mathrm{H}(112)$ & 0.9300 \\
\hline$C(111)-C(110)$ & $1.397(4)$ \\
\hline $\mathrm{C}(111)-\mathrm{H}(111)$ & 0.9300 \\
\hline $\mathrm{C}(110)-\mathrm{C}(19)$ & $1.390(3)$ \\
\hline $\mathrm{C}(110)-\mathrm{H}(110)$ & 0.9300 \\
\hline$C(19)-N(13)$ & $1.399(3)$ \\
\hline$N(13)-C(12)$ & $1.461(3)$ \\
\hline $\mathrm{C}(12)-\mathrm{H}(12 \mathrm{~A})$ & 0.9600 \\
\hline
\end{tabular}




\begin{tabular}{|c|c|}
\hline $\mathrm{C}(12)-\mathrm{H}(12 \mathrm{~B})$ & 0.9600 \\
\hline $\mathrm{C}(12)-\mathrm{H}(12 \mathrm{C})$ & 0.9600 \\
\hline $\mathrm{N}(14)-\mathrm{C}(118)$ & $1.306(3)$ \\
\hline $\mathrm{N}(14)-\mathrm{C}(117)$ & $1.313(3)$ \\
\hline $\mathrm{F}(11)-\mathrm{C}(116)$ & $1.353(3)$ \\
\hline $\mathrm{F}(12)-\mathrm{C}(117)$ & $1.350(3)$ \\
\hline $\mathrm{F}(13)-\mathrm{C}(118)$ & $1.348(3)$ \\
\hline $\mathrm{F}(14)-\mathrm{C}(119)$ & $1.348(3)$ \\
\hline$C(115)-C(116)$ & $1.391(3)$ \\
\hline$C(115)-C(119)$ & $1.393(3)$ \\
\hline$C(116)-C(117)$ & $1.370(3)$ \\
\hline $\mathrm{C}(118)-\mathrm{C}(119)$ & $1.379(3)$ \\
\hline $\mathrm{C}(21)-\mathrm{N}(22)$ & $1.461(3)$ \\
\hline$C(21)-H(21 A)$ & 0.9600 \\
\hline $\mathrm{C}(21)-\mathrm{H}(21 \mathrm{~B})$ & 0.9600 \\
\hline$C(21)-H(21 C)$ & 0.9600 \\
\hline$C(22)-N(23)$ & $1.460(3)$ \\
\hline $\mathrm{C}(22)-\mathrm{H}(22 \mathrm{~A})$ & 0.9600 \\
\hline$C(22)-H(22 B)$ & 0.9600 \\
\hline $\mathrm{C}(22)-\mathrm{H}(22 \mathrm{C})$ & 0.9600 \\
\hline$C(23)-C(24)$ & $1.380(3)$ \\
\hline$C(23)-N(22)$ & $1.402(3)$ \\
\hline$C(23)-C(28)$ & $1.410(3)$ \\
\hline$C(24)-C(25)$ & $1.400(3)$ \\
\hline $\mathrm{C}(24)-\mathrm{H}(24)$ & 0.9300 \\
\hline$C(25)-C(26)$ & $1.377(3)$ \\
\hline $\mathrm{C}(25)-\mathrm{H}(25)$ & 0.9300 \\
\hline$C(26)-C(27)$ & $1.404(3)$ \\
\hline $\mathrm{C}(26)-\mathrm{H}(26)$ & 0.9300 \\
\hline $\mathrm{C}(27)-\mathrm{C}(28)$ & $1.385(3)$ \\
\hline $\mathrm{C}(27)-\mathrm{H}(27)$ & 0.9300 \\
\hline $\mathrm{C}(28)-\mathrm{N}(21)$ & $1.401(3)$ \\
\hline$C(29)-C(210)$ & $1.379(3)$ \\
\hline$C(29)-N(23)$ & $1.400(3)$ \\
\hline$C(29)-C(214)$ & $1.415(3)$ \\
\hline$C(210)-C(211)$ & $1.395(3)$ \\
\hline $\mathrm{C}(210)-\mathrm{H}(210)$ & 0.9300 \\
\hline
\end{tabular}




\begin{tabular}{|c|c|}
\hline$C(211)-C(212)$ & $1.387(3)$ \\
\hline $\mathrm{C}(211)-\mathrm{H}(211)$ & 0.9300 \\
\hline$C(212)-C(213)$ & $1.400(3)$ \\
\hline $\mathrm{C}(212)-\mathrm{H}(212)$ & 0.9300 \\
\hline$C(213)-C(214)$ & $1.383(3)$ \\
\hline $\mathrm{C}(213)-\mathrm{H}(213)$ & 0.9300 \\
\hline $\mathrm{C}(214)-\mathrm{N}(21)$ & $1.397(3)$ \\
\hline$C(215)-C(219)$ & $1.394(3)$ \\
\hline$C(215)-C(216)$ & $1.396(3)$ \\
\hline$C(215)-P(2)$ & $1.878(3)$ \\
\hline$C(216)-F(21)$ & $1.349(3)$ \\
\hline$C(216)-C(217)$ & $1.370(4)$ \\
\hline$C(217)-N(24)$ & $1.318(3)$ \\
\hline $\mathrm{C}(217)-\mathrm{F}(22)$ & $1.347(3)$ \\
\hline $\mathrm{C}(218)-\mathrm{N}(24)$ & $1.316(3)$ \\
\hline$C(218)-F(23)$ & $1.347(3)$ \\
\hline$C(218)-C(219)$ & $1.370(4)$ \\
\hline$C(219)-F(24)$ & $1.356(3)$ \\
\hline$P(2)-N(23)$ & $1.688(2)$ \\
\hline$P(2)-N(22)$ & $1.691(2)$ \\
\hline$P(2)-N(21)$ & $1.7898(19)$ \\
\hline$P(2)-H(2)$ & $1.353(19)$ \\
\hline$N(13)-P(1)-N(12)$ & $139.41(10)$ \\
\hline$N(13)-P(1)-N(11)$ & $86.69(9)$ \\
\hline$N(12)-P(1)-N(11)$ & $87.01(9)$ \\
\hline$N(13)-P(1)-C(115)$ & $111.25(10)$ \\
\hline$N(12)-P(1)-C(115)$ & $109.19(10)$ \\
\hline$N(11)-P(1)-C(115)$ & $94.31(9)$ \\
\hline $\mathrm{N}(13)-\mathrm{P}(1)-\mathrm{H}(1)$ & $93.0(11)$ \\
\hline $\mathrm{N}(12)-\mathrm{P}(1)-\mathrm{H}(1)$ & $88.5(11)$ \\
\hline $\mathrm{N}(11)-\mathrm{P}(1)-\mathrm{H}(1)$ & $172.7(10)$ \\
\hline $\mathrm{C}(115)-\mathrm{P}(1)-\mathrm{H}(1)$ & $92.6(10)$ \\
\hline $\mathrm{N}(12)-\mathrm{C}(11)-\mathrm{H}(11 \mathrm{~A})$ & 109.5 \\
\hline $\mathrm{N}(12)-\mathrm{C}(11)-\mathrm{H}(11 \mathrm{~B})$ & 109.5 \\
\hline $\mathrm{H}(11 \mathrm{~A})-\mathrm{C}(11)-\mathrm{H}(11 \mathrm{~B})$ & 109.5 \\
\hline $\mathrm{N}(12)-\mathrm{C}(11)-\mathrm{H}(11 \mathrm{C})$ & 109.5 \\
\hline
\end{tabular}




\begin{tabular}{|c|c|}
\hline $\mathrm{H}(11 \mathrm{~A})-\mathrm{C}(11)-\mathrm{H}(11 \mathrm{C})$ & 109.5 \\
\hline $\mathrm{H}(11 \mathrm{~B})-\mathrm{C}(11)-\mathrm{H}(11 \mathrm{C})$ & 109.5 \\
\hline $\mathrm{C}(13)-\mathrm{N}(12)-\mathrm{C}(11)$ & $119.9(2)$ \\
\hline $\mathrm{C}(13)-\mathrm{N}(12)-\mathrm{P}(1)$ & $115.76(15)$ \\
\hline $\mathrm{C}(11)-\mathrm{N}(12)-\mathrm{P}(1)$ & $122.63(17)$ \\
\hline$C(14)-C(13)-C(18)$ & $120.8(2)$ \\
\hline$C(14)-C(13)-N(12)$ & $127.8(2)$ \\
\hline $\mathrm{C}(18)-\mathrm{C}(13)-\mathrm{N}(12)$ & $111.4(2)$ \\
\hline$C(13)-C(14)-C(15)$ & $118.8(2)$ \\
\hline $\mathrm{C}(13)-\mathrm{C}(14)-\mathrm{H}(14)$ & 120.6 \\
\hline $\mathrm{C}(15)-\mathrm{C}(14)-\mathrm{H}(14)$ & 120.6 \\
\hline$C(16)-C(15)-C(14)$ & $120.8(2)$ \\
\hline $\mathrm{C}(16)-\mathrm{C}(15)-\mathrm{H}(15)$ & 119.6 \\
\hline $\mathrm{C}(14)-\mathrm{C}(15)-\mathrm{H}(15)$ & 119.6 \\
\hline$C(15)-C(16)-C(17)$ & $120.7(2)$ \\
\hline $\mathrm{C}(15)-\mathrm{C}(16)-\mathrm{H}(16)$ & 119.6 \\
\hline$C(17)-C(16)-H(16)$ & 119.6 \\
\hline$C(18)-C(17)-C(16)$ & $118.6(2)$ \\
\hline $\mathrm{C}(18)-\mathrm{C}(17)-\mathrm{H}(17)$ & 120.7 \\
\hline $\mathrm{C}(16)-\mathrm{C}(17)-\mathrm{H}(17)$ & 120.7 \\
\hline$C(17)-C(18)-N(11)$ & $129.9(2)$ \\
\hline$C(17)-C(18)-C(13)$ & $120.3(2)$ \\
\hline$N(11)-C(18)-C(13)$ & $109.9(2)$ \\
\hline $\mathrm{C}(18)-\mathrm{N}(11)-\mathrm{C}(114)$ & $128.2(2)$ \\
\hline $\mathrm{C}(18)-\mathrm{N}(11)-\mathrm{P}(1)$ & $114.65(15)$ \\
\hline$C(114)-N(11)-P(1)$ & $114.52(16)$ \\
\hline$C(113)-C(114)-N(11)$ & $130.8(2)$ \\
\hline$C(113)-C(114)-C(19)$ & $120.4(2)$ \\
\hline $\mathrm{N}(11)-\mathrm{C}(114)-\mathrm{C}(19)$ & $108.8(2)$ \\
\hline $\mathrm{C}(114)-\mathrm{C}(113)-\mathrm{C}(112)$ & $118.9(2)$ \\
\hline $\mathrm{C}(114)-\mathrm{C}(113)-\mathrm{H}(113)$ & 120.5 \\
\hline $\mathrm{C}(112)-\mathrm{C}(113)-\mathrm{H}(113)$ & 120.5 \\
\hline$C(111)-C(112)-C(113)$ & $120.6(2)$ \\
\hline $\mathrm{C}(111)-\mathrm{C}(112)-\mathrm{H}(112)$ & 119.7 \\
\hline $\mathrm{C}(113)-\mathrm{C}(112)-\mathrm{H}(112)$ & 119.7 \\
\hline $\mathrm{C}(112)-\mathrm{C}(111)-\mathrm{C}(110)$ & $120.7(2)$ \\
\hline $\mathrm{C}(112)-\mathrm{C}(111)-\mathrm{H}(111)$ & 119.6 \\
\hline
\end{tabular}




\begin{tabular}{|c|c|}
\hline $\mathrm{C}(110)-\mathrm{C}(111)-\mathrm{H}(111)$ & 119.6 \\
\hline$C(19)-C(110)-C(111)$ & $118.7(2)$ \\
\hline $\mathrm{C}(19)-\mathrm{C}(110)-\mathrm{H}(110)$ & 120.6 \\
\hline $\mathrm{C}(111)-\mathrm{C}(110)-\mathrm{H}(110)$ & 120.6 \\
\hline$C(110)-C(19)-N(13)$ & $127.2(2)$ \\
\hline$C(110)-C(19)-C(114)$ & $120.6(2)$ \\
\hline$N(13)-C(19)-C(114)$ & $112.1(2)$ \\
\hline $\mathrm{C}(19)-\mathrm{N}(13)-\mathrm{C}(12)$ & $118.9(2)$ \\
\hline $\mathrm{C}(19)-\mathrm{N}(13)-\mathrm{P}(1)$ & $115.65(15)$ \\
\hline $\mathrm{C}(12)-\mathrm{N}(13)-\mathrm{P}(1)$ & $123.89(17)$ \\
\hline $\mathrm{N}(13)-\mathrm{C}(12)-\mathrm{H}(12 \mathrm{~A})$ & 109.5 \\
\hline $\mathrm{N}(13)-\mathrm{C}(12)-\mathrm{H}(12 \mathrm{~B})$ & 109.5 \\
\hline $\mathrm{H}(12 \mathrm{~A})-\mathrm{C}(12)-\mathrm{H}(12 \mathrm{~B})$ & 109.5 \\
\hline $\mathrm{N}(13)-\mathrm{C}(12)-\mathrm{H}(12 \mathrm{C})$ & 109.5 \\
\hline $\mathrm{H}(12 \mathrm{~A})-\mathrm{C}(12)-\mathrm{H}(12 \mathrm{C})$ & 109.5 \\
\hline $\mathrm{H}(12 \mathrm{~B})-\mathrm{C}(12)-\mathrm{H}(12 \mathrm{C})$ & 109.5 \\
\hline $\mathrm{C}(118)-\mathrm{N}(14)-\mathrm{C}(117)$ & $114.9(2)$ \\
\hline$C(116)-C(115)-C(119)$ & $113.1(2)$ \\
\hline$C(116)-C(115)-P(1)$ & $124.07(18)$ \\
\hline$C(119)-C(115)-P(1)$ & $122.67(18)$ \\
\hline $\mathrm{F}(11)-\mathrm{C}(116)-\mathrm{C}(117)$ & $117.4(2)$ \\
\hline$F(11)-C(116)-C(115)$ & $121.4(2)$ \\
\hline$C(117)-C(116)-C(115)$ & $121.2(2)$ \\
\hline$N(14)-C(117)-F(12)$ & $116.2(2)$ \\
\hline $\mathrm{N}(14)-\mathrm{C}(117)-\mathrm{C}(116)$ & $124.9(2)$ \\
\hline $\mathrm{F}(12)-\mathrm{C}(117)-\mathrm{C}(116)$ & $118.9(2)$ \\
\hline $\mathrm{N}(14)-\mathrm{C}(118)-\mathrm{F}(13)$ & $116.1(2)$ \\
\hline $\mathrm{N}(14)-\mathrm{C}(118)-\mathrm{C}(119)$ & $125.0(2)$ \\
\hline $\mathrm{F}(13)-\mathrm{C}(118)-\mathrm{C}(119)$ & $118.9(2)$ \\
\hline $\mathrm{F}(14)-\mathrm{C}(119)-\mathrm{C}(118)$ & $117.5(2)$ \\
\hline $\mathrm{F}(14)-\mathrm{C}(119)-\mathrm{C}(115)$ & $121.6(2)$ \\
\hline$C(118)-C(119)-C(115)$ & $120.9(2)$ \\
\hline $\mathrm{N}(22)-\mathrm{C}(21)-\mathrm{H}(21 \mathrm{~A})$ & 109.5 \\
\hline $\mathrm{N}(22)-\mathrm{C}(21)-\mathrm{H}(21 \mathrm{~B})$ & 109.5 \\
\hline $\mathrm{H}(21 \mathrm{~A})-\mathrm{C}(21)-\mathrm{H}(21 \mathrm{~B})$ & 109.5 \\
\hline $\mathrm{N}(22)-\mathrm{C}(21)-\mathrm{H}(21 \mathrm{C})$ & 109.5 \\
\hline $\mathrm{H}(21 \mathrm{~A})-\mathrm{C}(21)-\mathrm{H}(21 \mathrm{C})$ & 109.5 \\
\hline
\end{tabular}




\begin{tabular}{|c|c|}
\hline $\mathrm{H}(21 \mathrm{~B})-\mathrm{C}(21)-\mathrm{H}(21 \mathrm{C})$ & 109.5 \\
\hline $\mathrm{N}(23)-\mathrm{C}(22)-\mathrm{H}(22 \mathrm{~A})$ & 109.5 \\
\hline $\mathrm{N}(23)-\mathrm{C}(22)-\mathrm{H}(22 \mathrm{~B})$ & 109.5 \\
\hline $\mathrm{H}(22 \mathrm{~A})-\mathrm{C}(22)-\mathrm{H}(22 \mathrm{~B})$ & 109.5 \\
\hline $\mathrm{N}(23)-\mathrm{C}(22)-\mathrm{H}(22 \mathrm{C})$ & 109.5 \\
\hline $\mathrm{H}(22 \mathrm{~A})-\mathrm{C}(22)-\mathrm{H}(22 \mathrm{C})$ & 109.5 \\
\hline $\mathrm{H}(22 \mathrm{~B})-\mathrm{C}(22)-\mathrm{H}(22 \mathrm{C})$ & 109.5 \\
\hline$C(24)-C(23)-N(22)$ & $127.5(2)$ \\
\hline$C(24)-C(23)-C(28)$ & $121.0(2)$ \\
\hline $\mathrm{N}(22)-\mathrm{C}(23)-\mathrm{C}(28)$ & $111.5(2)$ \\
\hline$C(23)-C(24)-C(25)$ & $118.6(2)$ \\
\hline $\mathrm{C}(23)-\mathrm{C}(24)-\mathrm{H}(24)$ & 120.7 \\
\hline $\mathrm{C}(25)-\mathrm{C}(24)-\mathrm{H}(24)$ & 120.7 \\
\hline$C(26)-C(25)-C(24)$ & $120.7(2)$ \\
\hline $\mathrm{C}(26)-\mathrm{C}(25)-\mathrm{H}(25)$ & 119.6 \\
\hline $\mathrm{C}(24)-\mathrm{C}(25)-\mathrm{H}(25)$ & 119.6 \\
\hline$C(25)-C(26)-C(27)$ & $120.9(2)$ \\
\hline$C(25)-C(26)-H(26)$ & 119.5 \\
\hline $\mathrm{C}(27)-\mathrm{C}(26)-\mathrm{H}(26)$ & 119.5 \\
\hline$C(28)-C(27)-C(26)$ & $118.7(2)$ \\
\hline $\mathrm{C}(28)-\mathrm{C}(27)-\mathrm{H}(27)$ & 120.7 \\
\hline $\mathrm{C}(26)-\mathrm{C}(27)-\mathrm{H}(27)$ & 120.7 \\
\hline$C(27)-C(28)-N(21)$ & $130.6(2)$ \\
\hline$C(27)-C(28)-C(23)$ & $120.0(2)$ \\
\hline $\mathrm{N}(21)-\mathrm{C}(28)-\mathrm{C}(23)$ & $109.44(19)$ \\
\hline$C(210)-C(29)-N(23)$ & $127.7(2)$ \\
\hline$C(210)-C(29)-C(214)$ & $121.0(2)$ \\
\hline$N(23)-C(29)-C(214)$ & $111.4(2)$ \\
\hline$C(29)-C(210)-C(211)$ & $119.1(2)$ \\
\hline $\mathrm{C}(29)-\mathrm{C}(210)-\mathrm{H}(210)$ & 120.4 \\
\hline $\mathrm{C}(211)-\mathrm{C}(210)-\mathrm{H}(210)$ & 120.4 \\
\hline$C(212)-C(211)-C(210)$ & $120.1(2)$ \\
\hline $\mathrm{C}(212)-\mathrm{C}(211)-\mathrm{H}(211)$ & 119.9 \\
\hline$C(210)-C(211)-H(211)$ & 119.9 \\
\hline$C(211)-C(212)-C(213)$ & $121.1(2)$ \\
\hline$C(211)-C(212)-H(212)$ & 119.5 \\
\hline$C(213)-C(212)-H(212)$ & 119.5 \\
\hline
\end{tabular}




\begin{tabular}{|c|c|}
\hline$C(214)-C(213)-C(212)$ & $119.0(2)$ \\
\hline $\mathrm{C}(214)-\mathrm{C}(213)-\mathrm{H}(213)$ & 120.5 \\
\hline$C(212)-C(213)-H(213)$ & 120.5 \\
\hline $\mathrm{C}(213)-\mathrm{C}(214)-\mathrm{N}(21)$ & $131.1(2)$ \\
\hline$C(213)-C(214)-C(29)$ & $119.7(2)$ \\
\hline $\mathrm{N}(21)-\mathrm{C}(214)-\mathrm{C}(29)$ & $109.22(19)$ \\
\hline$C(219)-C(215)-C(216)$ & $113.0(2)$ \\
\hline$C(219)-C(215)-P(2)$ & $123.68(19)$ \\
\hline$C(216)-C(215)-P(2)$ & $123.29(18)$ \\
\hline$F(21)-C(216)-C(217)$ & $117.3(2)$ \\
\hline$F(21)-C(216)-C(215)$ & $121.6(2)$ \\
\hline$C(217)-C(216)-C(215)$ & $121.1(2)$ \\
\hline $\mathrm{N}(24)-\mathrm{C}(217)-\mathrm{F}(22)$ & $115.9(2)$ \\
\hline $\mathrm{N}(24)-\mathrm{C}(217)-\mathrm{C}(216)$ & $124.8(2)$ \\
\hline $\mathrm{F}(22)-\mathrm{C}(217)-\mathrm{C}(216)$ & $119.3(2)$ \\
\hline $\mathrm{N}(24)-\mathrm{C}(218)-\mathrm{F}(23)$ & $115.6(2)$ \\
\hline $\mathrm{N}(24)-\mathrm{C}(218)-\mathrm{C}(219)$ & $124.7(2)$ \\
\hline $\mathrm{F}(23)-\mathrm{C}(218)-\mathrm{C}(219)$ & $119.7(2)$ \\
\hline $\mathrm{F}(24)-\mathrm{C}(219)-\mathrm{C}(218)$ & $117.6(2)$ \\
\hline$F(24)-C(219)-C(215)$ & $121.0(2)$ \\
\hline$C(218)-C(219)-C(215)$ & $121.4(2)$ \\
\hline$N(23)-P(2)-N(22)$ & $141.92(11)$ \\
\hline$N(23)-P(2)-N(21)$ & $86.53(9)$ \\
\hline$N(22)-P(2)-N(21)$ & $86.70(9)$ \\
\hline$N(23)-P(2)-C(215)$ & $109.31(11)$ \\
\hline$N(22)-P(2)-C(215)$ & $108.65(11)$ \\
\hline$N(21)-P(2)-C(215)$ & $95.95(10)$ \\
\hline $\mathrm{N}(23)-\mathrm{P}(2)-\mathrm{H}(2)$ & $91.9(11)$ \\
\hline $\mathrm{N}(22)-\mathrm{P}(2)-\mathrm{H}(2)$ & $89.3(11)$ \\
\hline $\mathrm{N}(21)-\mathrm{P}(2)-\mathrm{H}(2)$ & $171.4(11)$ \\
\hline$C(215)-P(2)-H(2)$ & $92.6(11)$ \\
\hline$C(214)-N(21)-C(28)$ & $128.89(19)$ \\
\hline$C(214)-N(21)-P(2)$ & $114.24(15)$ \\
\hline $\mathrm{C}(28)-\mathrm{N}(21)-\mathrm{P}(2)$ & $114.64(14)$ \\
\hline$C(23)-N(22)-C(21)$ & $118.79(19)$ \\
\hline $\mathrm{C}(23)-\mathrm{N}(22)-\mathrm{P}(2)$ & $116.62(15)$ \\
\hline $\mathrm{C}(21)-\mathrm{N}(22)-\mathrm{P}(2)$ & $123.13(16)$ \\
\hline
\end{tabular}




$\begin{array}{ll}\mathrm{C}(29)-\mathrm{N}(23)-\mathrm{C}(22) & 119.01(19) \\ \mathrm{C}(29)-\mathrm{N}(23)-\mathrm{P}(2) & 116.16(15) \\ \mathrm{C}(22)-\mathrm{N}(23)-\mathrm{P}(2) & 124.19(15) \\ \mathrm{C}(218)-\mathrm{N}(24)-\mathrm{C}(217) & 114.9(2)\end{array}$

Symmetry transformations used to generate equivalent atoms: 
Table S19. Anisotropic displacement parameters $\left(\AA^{2} \times 10^{3}\right)$ for $\mathbf{1} \cdot[\mathrm{H}]\left[\mathrm{C}_{5} \mathrm{~F}_{4} \mathrm{~N}\right]$. The anisotropic displacement factor exponent takes the form: $-2 \mathrm{p}^{2}\left[\mathrm{~h}^{2} \mathrm{a}^{* 2} \mathrm{U}^{11}+\ldots+2 \mathrm{hka} \mathrm{b}^{*} \mathrm{U}^{12}\right]$

\begin{tabular}{|c|c|c|c|c|c|c|}
\hline & $\mathrm{U}^{11}$ & $\mathrm{U}^{22}$ & $\mathrm{U}^{33}$ & $\mathrm{U}^{23}$ & $\mathrm{U}^{13}$ & $\mathrm{U}^{12}$ \\
\hline$P(1)$ & $20(1)$ & $10(1)$ & $12(1)$ & $0(1)$ & $4(1)$ & $0(1)$ \\
\hline$C(11)$ & $26(2)$ & $16(1)$ & $16(1)$ & $0(1)$ & $9(1)$ & $2(1)$ \\
\hline$N(12)$ & $20(1)$ & $10(1)$ & $16(1)$ & $-1(1)$ & $7(1)$ & $0(1)$ \\
\hline$C(13)$ & $20(1)$ & $14(1)$ & $10(1)$ & $1(1)$ & $4(1)$ & $-2(1)$ \\
\hline$C(14)$ & $24(2)$ & $12(1)$ & $16(1)$ & $0(1)$ & $6(1)$ & $3(1)$ \\
\hline$C(15)$ & $24(2)$ & $10(1)$ & $19(1)$ & $2(1)$ & $3(1)$ & $0(1)$ \\
\hline$C(16)$ & $20(1)$ & $14(1)$ & $16(1)$ & $2(1)$ & $4(1)$ & $-3(1)$ \\
\hline $\mathrm{C}(17)$ & $22(2)$ & $12(1)$ & $12(1)$ & $0(1)$ & $4(1)$ & $1(1)$ \\
\hline$C(18)$ & $20(1)$ & $11(1)$ & $10(1)$ & $1(1)$ & $1(1)$ & $1(1)$ \\
\hline $\mathrm{N}(11)$ & $21(1)$ & $6(1)$ & $14(1)$ & $0(1)$ & $5(1)$ & $1(1)$ \\
\hline $\mathrm{C}(114)$ & $22(1)$ & $9(1)$ & $11(1)$ & $0(1)$ & $2(1)$ & $2(1)$ \\
\hline$C(113)$ & $24(2)$ & $13(1)$ & $11(1)$ & $0(1)$ & $4(1)$ & $-1(1)$ \\
\hline $\mathrm{C}(112)$ & $20(1)$ & $16(1)$ & $17(1)$ & $-4(1)$ & $5(1)$ & $1(1)$ \\
\hline $\mathrm{C}(111)$ & $26(2)$ & $12(1)$ & $20(1)$ & $-2(1)$ & $4(1)$ & $3(1)$ \\
\hline $\mathrm{C}(110)$ & $26(2)$ & $10(1)$ & $17(1)$ & $0(1)$ & $3(1)$ & $-2(1)$ \\
\hline$C(19)$ & $20(1)$ & $11(1)$ & $12(1)$ & $-1(1)$ & $0(1)$ & $0(1)$ \\
\hline $\mathrm{N}(13)$ & $18(1)$ & $11(1)$ & $14(1)$ & $2(1)$ & $5(1)$ & $-2(1)$ \\
\hline$C(12)$ & $26(2)$ & $11(1)$ & $19(1)$ & $4(1)$ & $7(1)$ & $-2(1)$ \\
\hline $\mathrm{N}(14)$ & $20(1)$ & $18(1)$ & $15(1)$ & $1(1)$ & $6(1)$ & $-1(1)$ \\
\hline $\mathrm{F}(11)$ & $35(1)$ & $9(1)$ & $18(1)$ & $0(1)$ & $6(1)$ & $3(1)$ \\
\hline$F(12)$ & $30(1)$ & $17(1)$ & $17(1)$ & $6(1)$ & $4(1)$ & $5(1)$ \\
\hline$F(13)$ & $29(1)$ & $16(1)$ & $17(1)$ & $-6(1)$ & $6(1)$ & $-3(1)$ \\
\hline $\mathrm{F}(14)$ & $28(1)$ & $10(1)$ & $18(1)$ & $-1(1)$ & $4(1)$ & $2(1)$ \\
\hline$C(115)$ & $15(1)$ & $11(1)$ & $15(1)$ & $1(1)$ & $6(1)$ & $-2(1)$ \\
\hline$C(116)$ & $22(2)$ & $12(1)$ & $18(1)$ & $-3(1)$ & $6(1)$ & $-2(1)$ \\
\hline $\mathrm{C}(117)$ & $18(1)$ & $15(1)$ & $17(1)$ & $5(1)$ & $7(1)$ & $3(1)$ \\
\hline $\mathrm{C}(118)$ & $22(2)$ & $14(1)$ & $18(1)$ & $-4(1)$ & $9(1)$ & $-3(1)$ \\
\hline $\mathrm{C}(119)$ & $17(1)$ & $12(1)$ & $17(1)$ & $2(1)$ & $6(1)$ & $1(1)$ \\
\hline$C(21)$ & $27(2)$ & $15(1)$ & $14(1)$ & $-1(1)$ & $7(1)$ & $0(1)$ \\
\hline$C(22)$ & $25(2)$ & $13(1)$ & $16(1)$ & $2(1)$ & $5(1)$ & $0(1)$ \\
\hline$C(23)$ & $16(1)$ & $16(1)$ & $15(1)$ & $2(1)$ & $5(1)$ & $0(1)$ \\
\hline \multirow[t]{2}{*}{$C(24)$} & $20(1)$ & $14(1)$ & $17(1)$ & $-3(1)$ & $7(1)$ & $-2(1)$ \\
\hline & & & & S57 & & \\
\hline
\end{tabular}




$\begin{array}{lcccccc}\mathrm{C}(25) & 21(2) & 11(1) & 25(1) & 2(1) & 9(1) & 2(1) \\ \mathrm{C}(26) & 21(2) & 15(1) & 18(1) & 4(1) & 5(1) & 1(1) \\ \mathrm{C}(27) & 21(2) & 13(1) & 16(1) & 1(1) & 6(1) & 2(1) \\ \mathrm{C}(28) & 19(1) & 10(1) & 18(1) & 0(1) & 7(1) & -1(1) \\ \mathrm{C}(29) & 19(1) & 16(1) & 15(1) & 1(1) & 6(1) & -1(1) \\ \mathrm{C}(210) & 19(1) & 10(1) & 21(1) & 3(1) & 5(1) & 1(1) \\ \mathrm{C}(211) & 21(2) & 11(1) & 21(1) & -3(1) & 7(1) & -2(1) \\ \mathrm{C}(212) & 21(1) & 16(1) & 14(1) & 0(1) & 4(1) & -1(1) \\ \mathrm{C}(213) & 21(1) & 13(1) & 16(1) & 2(1) & 6(1) & 1(1) \\ \mathrm{C}(214) & 17(1) & 9(1) & 18(1) & -1(1) & 6(1) & 0(1) \\ \mathrm{C}(215) & 23(1) & 11(1) & 10(1) & 2(1) & 3(1) & 1(1) \\ \mathrm{C}(216) & 22(2) & 9(1) & 15(1) & 0(1) & 5(1) & 3(1) \\ \mathrm{C}(217) & 23(2) & 11(1) & 14(1) & 0(1) & 4(1) & -3(1) \\ \mathrm{C}(218) & 23(2) & 12(1) & 14(1) & -2(1) & 5(1) & 4(1) \\ \mathrm{C}(219) & 24(2) & 8(1) & 16(1) & -2(1) & 4(1) & -1(1) \\ \mathrm{P}(2) & 20(1) & 12(1) & 12(1) & 1(1) & 4(1) & 1(1) \\ \mathrm{N}(21) & 20(1) & 10(1) & 13(1) & 1(1) & 4(1) & 1(1) \\ \mathrm{N}(22) & 20(1) & 13(1) & 13(1) & 1(1) & 4(1) & 2(1) \\ \mathrm{N}(23) & 21(1) & 12(1) & 12(1) & 3(1) & 4(1) & 0(1) \\ \mathrm{N}(24) & 21(1) & 15(1) & 14(1) & -1(1) & 5(1) & 0(1) \\ \mathrm{F}(21) & 26(1) & 9(1) & 28(1) & 0(1) & 11(1) & 2(1) \\ \mathrm{F}(22) & 26(1) & 11(1) & 25(1) & -1(1) & 9(1) & -4(1) \\ \mathrm{F}(23) & 25(1) & 15(1) & 26(1) & -5(1) & 9(1) & 2(1) \\ \mathrm{F}(24) & 26(1) & 8(1) & 27(1) & -2(1) & 9(1) & -1(1) \\ & & & & & & \\ \end{array}$


Table S20. Hydrogen coordinates $\left(\times 10^{4}\right)$ and isotropic displacement parameters $\left(\AA^{2} \times 10^{3}\right)$ for $\mathbf{1} \cdot[\mathrm{H}]\left[\mathrm{C}_{5} \mathrm{~F}_{4} \mathrm{~N}\right]$.

\begin{tabular}{|c|c|c|c|c|}
\hline & $\mathrm{x}$ & $\mathrm{y}$ & $\mathrm{z}$ & $\mathrm{U}(\mathrm{eq})$ \\
\hline $\mathrm{H}(1)$ & $7970(20)$ & $6492(7)$ & $2870(30)$ & 17 \\
\hline $\mathrm{H}(11 \mathrm{~A})$ & 8331 & 5715 & 3039 & 29 \\
\hline $\mathrm{H}(11 \mathrm{~B})$ & 8222 & 6027 & 1715 & 29 \\
\hline $\mathrm{H}(11 \mathrm{C})$ & 7401 & 5685 & 1184 & 29 \\
\hline $\mathrm{H}(14)$ & 6272 & 5306 & 2350 & 21 \\
\hline $\mathrm{H}(15)$ & 4706 & 5044 & 3064 & 22 \\
\hline $\mathrm{H}(16)$ & 3471 & 5393 & 4091 & 20 \\
\hline $\mathrm{H}(17)$ & 3820 & 6011 & 4538 & 19 \\
\hline $\mathrm{H}(113)$ & 3737 & 6571 & 4961 & 20 \\
\hline $\mathrm{H}(112)$ & 2878 & 7146 & 4658 & 22 \\
\hline $\mathrm{H}(111)$ & 3635 & 7608 & 3430 & 25 \\
\hline $\mathrm{H}(110)$ & 5299 & 7512 & 2548 & 22 \\
\hline $\mathrm{H}(12 \mathrm{~A})$ & 6607 & 7263 & 1154 & 28 \\
\hline $\mathrm{H}(12 \mathrm{~B})$ & 7767 & 7025 & 1799 & 28 \\
\hline $\mathrm{H}(12 \mathrm{C})$ & 7505 & 7315 & 3018 & 28 \\
\hline $\mathrm{H}(21 \mathrm{~A})$ & 2811 & 6778 & 10745 & 28 \\
\hline $\mathrm{H}(21 \mathrm{~B})$ & 2583 & 6400 & 11441 & 28 \\
\hline $\mathrm{H}(21 \mathrm{C})$ & 1526 & 6677 & 10775 & 28 \\
\hline $\mathrm{H}(22 \mathrm{~A})$ & 1469 & 5175 & 9021 & 28 \\
\hline $\mathrm{H}(22 \mathrm{~B})$ & 2426 & 5411 & 10375 & 28 \\
\hline $\mathrm{H}(22 \mathrm{C})$ & 2827 & 5149 & 9166 & 28 \\
\hline $\mathrm{H}(24)$ & 1242 & 7139 & 8525 & 20 \\
\hline $\mathrm{H}(25)$ & 358 & 7436 & 5918 & 23 \\
\hline $\mathrm{H}(26)$ & 7 & 7135 & 3367 & 22 \\
\hline $\mathrm{H}(27)$ & 613 & 6535 & 3328 & 20 \\
\hline $\mathrm{H}(210)$ & 1592 & 4964 & 6201 & 20 \\
\hline $\mathrm{H}(211)$ & 904 & 4909 & 3221 & 21 \\
\hline $\mathrm{H}(212)$ & 536 & 5419 & 1515 & 21 \\
\hline $\mathrm{H}(213)$ & 908 & 5993 & 2733 & 20 \\
\hline $\mathrm{H}(2)$ & $2840(20)$ & $5957(7)$ & $10180(20)$ & 18 \\
\hline
\end{tabular}




\section{Computational Details}

\section{a) General Computational Information}

Geometries were optimized in Orca 4.2.014 using the $\omega$ B97X-D3 density functional ${ }^{15}$ with the def2TZVP basis set. ${ }^{16}$ The choice of functional was based on a survey of several functionals and comparing optimized geometries with experimental crystallographic structures. Calculations were first carried out in the gas phase without symmetry constraint, and solvent correction was applied afterwards if necessary. Stationary points were characterized by frequency calculations to confirm their identity as local minima (ground states) or first-order saddle points (transition states). Natural bond order (NBO) analysis was performed with the NBO 6.0 program $^{17-19}$ through Orca. Molecular orbitals were visualized using the Jmol software. ${ }^{20}$ Intrinsic bond orbital (IBO) analysis was performed with the IboView software. ${ }^{21,22}$ Kinetic isotope effects (KIE) were computed by the KINISOT software. ${ }^{23}$

\section{b) Reaction Mechanism for Reductive Elimination}

Based on the ground state geometry of $\mathbf{1} \cdot[\mathrm{H}]\left[\mathrm{C}_{5} \mathrm{~F}_{4} \mathrm{E}\right]$, axial-equatorial coupling through the interaction between the $\mathrm{P}-\mathrm{H} \sigma$ and the aromatic $\pi^{*}$ of the fluoroaryl group was first postulated. The corresponding optimized saddle point (TS1) when $\mathrm{E}=\mathrm{N}$ has a single imaginary vibrational frequency $\left(-834.45 \mathrm{~cm}^{-1}\right)$ and was validated by the Intrinsic Reaction Coordinate method (IRC).24

(a) Optimized geometry of TS1

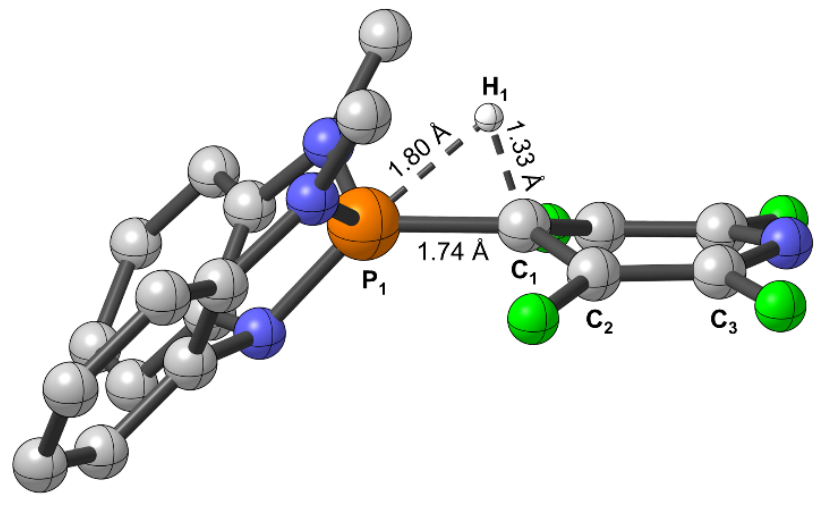

(b) HOMO of TS1

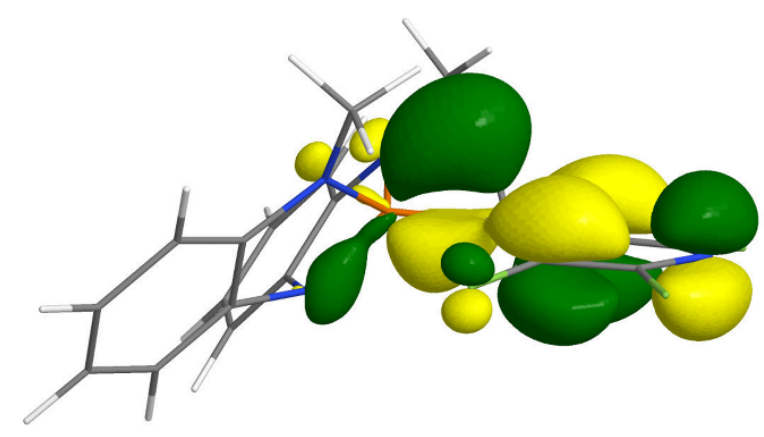

Figure S13. (a) The structure of TS1 with computed metrics ( $\omega B 97 X-D 3 / D e f 2-T Z V P)$. Hydrogen atoms except $\mathrm{H}_{1}$ are omitted for clarity. (b) The HOMO of the TS1.

In TS1, the hydride that was bonded to the phosphorus migrates to the fluoroaryl group with concomitant dearomatization (Figure S13a) with the predicted KIE value of 1.18. The HOMO of TS1 exhibits evident involvement of the aromatic $\pi^{*}$ orbital as well as conversion of the $\mathrm{H}_{1}-\mathrm{P}_{1}-\mathrm{N}_{1}$ 3center-4-electron bond into a $\mathrm{C}_{1}-\mathrm{H}_{1}-\mathrm{P}_{1} 3$-center-2-electron bond (Figure $\mathrm{S} 13 \mathrm{~b}$ ). Reflecting these 
orbital interactions, second-order perturbation theory in NBO basis predicts considerable interaction between $\mathrm{C}_{1}-\mathrm{H}_{1} \sigma$ and $\mathrm{P}_{1}-\mathrm{N}_{1} \sigma^{*}(31.0 \mathrm{kcal} / \mathrm{mol})$ and between $\mathrm{C}_{2}-\mathrm{C}_{3} \pi$ and $\mathrm{C}_{1}-\mathrm{H}_{1} \sigma^{*}(12.8$ $\mathrm{kcal} / \mathrm{mol})$.

Along the reaction coordinate (Figure 5 in the main manuscript), $\mathrm{P}_{1}-\mathrm{H}_{1}$ bond cleavage and $\mathrm{C}_{1}-\mathrm{H}_{1}$ bond formation occur simultaneously as the $\mathrm{H}_{1}$ atom moves from the phosphorus center to the fluoroaryl group, with the Wiberg bond order in TS1 being 0.538 for $\mathrm{C}_{1}-\mathrm{H}_{1}$ and 0.211 for $\mathrm{P}_{1}-\mathrm{H}_{1}$. Natural population analysis reveals the accumulation of partial negative charge on $\mathrm{H}_{1}(-0.139$ in 1•[H] $\left[\mathrm{C}_{5} \mathrm{~F}_{4} \mathrm{~N}\right]$ to -0.163 ) during the early phase of the reaction. Counterintuitively, the $\mathrm{P}_{1}-\mathrm{C}_{1}$ bond order increases near the saddle point as the $\mathrm{P}_{1}$ atom is rehybridized from $s p^{3.06}\left(\mathbf{1} \cdot[\mathrm{H}]\left[\mathrm{C}_{5} \mathrm{~F}_{4} \mathrm{~N}\right]\right)$ to $s p^{2.45}$ (TS1), although no stationary point corresponding to a stable phosphonium intermediate is obtained and geometry optimization of structures onward of TS1 along the intrinsic reaction coordinate invariably relax to products $\mathbf{1}$ and 3a. Electron flow near the saddle point in IBO basis (attached as a separate SI file) depicts the transfer of $\mathrm{H}_{1}$ from $\mathrm{P}_{1}$ to the fluoroaryl group entailing dearomatization.

Since the computed activation barrier for TS1 $\left(\Delta G^{\ddagger}=+49.2 \mathrm{kcal} / \mathrm{mol}\right)$ is too high even considering the reaction temperature above $140^{\circ} \mathrm{C}$, we sought after an alternative and wound up with the second pathway in which the fluoroaryl group becomes partially dissociated and subsequently abstracts the proton on the phosphorus. Again, the optimized transition state structure (TS2) when E = N has only one imaginary vibrational mode $\left(-50.0 \mathrm{~cm}^{-1}\right)$ and was validated by the IRC. The activation barrier is $+37.7 \mathrm{kcal} / \mathrm{mol}$, consistent with the reaction temperature.

In TS2, the fluoroaryl group is separated from the phosphorus with its planarity retained in contrast to the TS1, while the proton is still tightly bonded to the phosphorus (Figure S14a). The $\mathrm{P}_{1}-\mathrm{H}_{1}$ bond length remains same with the starting material (Table S21). Accordingly, the computed KIE $k_{\mathrm{H}} / k_{\mathrm{D}}=$ 1.02 was smaller than in TS1. The HOMO suggests high lone pair electron density on the $\mathrm{C}_{1}$, rendering a partial anionic character to the $\mathrm{C}_{5} \mathrm{~F}_{4} \mathrm{~N}$ moiety (Figure S14b). Indeed, a second-order perturbation analysis on the TS2 in NBO basis predicts significant donor-acceptor interaction $(17.3 \mathrm{kcal} / \mathrm{mol})$ between the $\mathrm{C}_{1}$ lone pair and the $\mathrm{P}_{1}-\mathrm{H}_{1} \sigma^{*}$. Nonetheless, the complete dissociation into an ion pair is energetically inaccessible in benzene $(\Delta G=+46.0 \mathrm{kcal} / \mathrm{mol})$, preventing intermolecular reactivity. 
(a) Optimized geometry of TS2

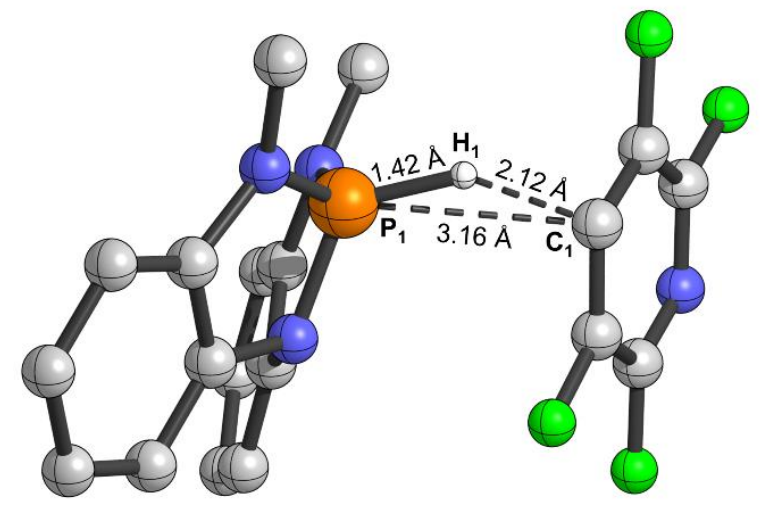

(b) HOMO of TS2

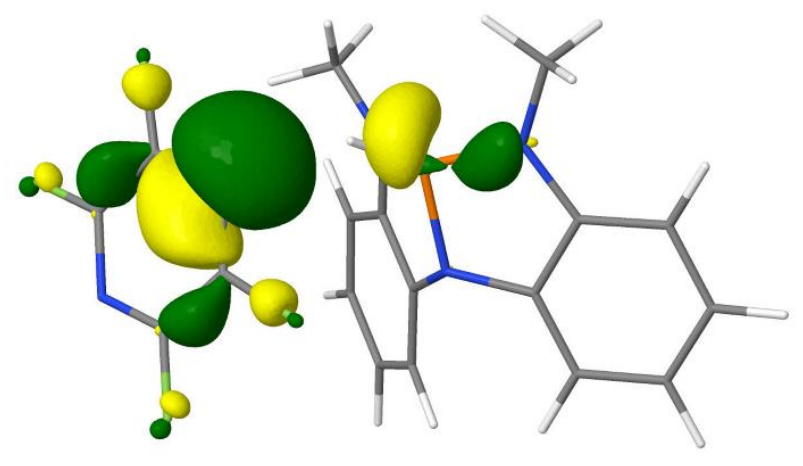

Figure S14. (a) The structure of TS2 with computed metrics ( $\omega$ B97X-D3/Def2-TZVP). Hydrogen atoms except $\mathrm{H}_{1}$ are omitted for clarity. (b) The HOMO of the TS2.

Table S21. Computed Distances $(\AA)$ for 1•[H] $\left[\mathrm{C}_{5} \mathrm{~F}_{4} \mathrm{~N}\right]$, TS2, 1, and 3a. Refer to Figure S14 for the atom numbering scheme.

\begin{tabular}{|c|cccc|}
\hline Metric & $\mathbf{1 \cdot}[\mathbf{H}]\left[\mathbf{C}_{5} \mathbf{F}_{4} \mathbf{N}\right]$ & TS2 & $\mathbf{1}$ & 3a \\
\hline$d\left(\mathrm{P}_{1}-\mathrm{H}_{1}\right)$ & 1.423 & 1.423 & - & - \\
$d\left(\mathrm{P}_{1}-\mathrm{C}_{1}\right)$ & 1.871 & 3.163 & - & - \\
$d\left(\mathrm{C}_{1}-\mathrm{H}_{1}\right)$ & 2.408 & 2.118 & - & 1.082 \\
\hline
\end{tabular}

Details of the full IRC path including natural charges and bond orders from the NBO analysis are plotted in Figure S15. It is found that while the $\mathrm{C}_{5} \mathrm{~F}_{4} \mathrm{~N}$ is dissociating from the phosphorus, there is no weakening of the $\mathrm{P}_{1}-\mathrm{H}_{1}$ bond until passing the saddle point. In the same vein, the hydrogen remains hydridic before reaching the saddle point, and turns protic thence. The charge plot also reveals that the negative charge accumulated on the $\mathrm{C}_{5} \mathrm{~F}_{4} \mathrm{~N}$ group with the heterolytic dissociation is not concentrated on the $\mathrm{C}_{1}$ but rather spread owing to its high electronegativity. Electron flow in IBO basis (attached as a separate SI file) clearly visualizes the highly asynchronous manner of this mechanism. Unlike in the first path, no noticeable changes in the aromatic $\pi^{*}$ orbitals of the fluoroaryl group is observed. 


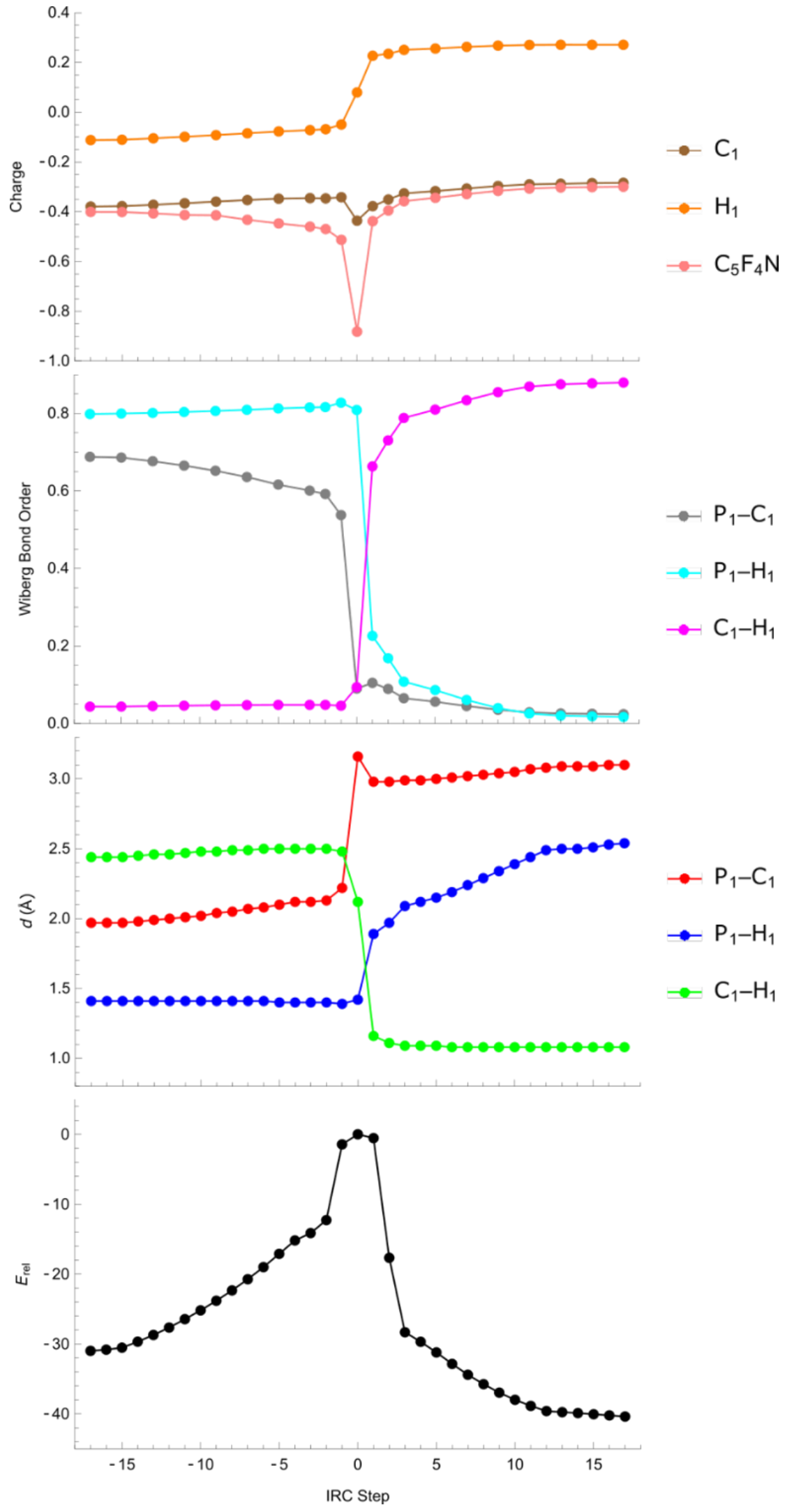

Figure S15. Computed relative energies, distances, Wiberg bond orders, and charges along the reaction coordinate. Step 0 indicates TS2. Negative steps are toward the reactant side $\left(\mathbf{1} \bullet[\mathrm{H}]\left[\mathrm{C}_{5} \mathrm{~F}_{4} \mathrm{~N}\right]\right)$ and positive steps are toward the product side $(\mathbf{1}+\mathbf{3 a})$. Refer to Figure S14 for the atom numbering scheme. 


\section{c) Energies and Cartesian Coordinates of Stationary Points}

The absolute thermochemical data with the unit of Hartrees and cartesian coordinates with the unit of $\AA$ for the $\omega$ B97X-D3/def2-TZVP stationary points in the gas phase at $298.15 \mathrm{~K}$ at $1.00 \mathrm{~atm}$.

1

Electronic Energy $=-1047.61168537$

Total Thermal Energy $=-1047.33840555$

Enthalpy $=-1047.33746134$

Free Energy $=-1047.39397023$

\begin{tabular}{llcc}
\hline C & 1.89702494255859 & 7.65687596065294 & 5.16539411750732 \\
C & 1.09530822399776 & 8.49163461988777 & 5.91303656179796 \\
H & 0.18367598731029 & 8.89836707572979 & 5.49864320873066 \\
C & 1.47168760653898 & 8.80370324895447 & 7.21957014058988 \\
H & 0.84656899356078 & 9.46192752120328 & 7.80991141778851 \\
C & 2.62644066358847 & 8.27324377770633 & 7.76319649699135 \\
H & 2.90707598015483 & 8.51660633877217 & 8.78075830928478 \\
C & 3.43628274310849 & 7.42031797577298 & 7.01551338505089 \\
H & 4.34387452910744 & 7.00969318400764 & 7.44046404735115 \\
C & 3.06154797833685 & 7.11464759491909 & 5.72052473907704 \\
C & 1.58201384623818 & 8.27331801971072 & 2.83351087968210 \\
C & 2.63260661011527 & 8.23932750234514 & 1.90719543485584 \\
C & 2.71086982667402 & 9.18603716945897 & 0.90201711584487 \\
H & 3.52808788460948 & 9.17366072941206 & 0.19151701662399 \\
C & 1.71805410185288 & 10.16188357532189 & 0.82167291344812 \\
H & 1.76889158244782 & 10.90529302350572 & 0.03523585253861 \\
C & 0.67018183757577 & 10.18227281191170 & 1.72201821512730 \\
H & -0.10198512070409 & 10.93686041493947 & 1.64073345564135 \\
C & 0.59310202469989 & 9.22534316784881 & 2.73524341680193 \\
H & -0.24241740034292 & 9.22291668974535 & 3.42164027775624 \\
C & 4.39611242380076 & 6.72096938443513 & 1.08879500959972 \\
H & 3.92963250866473 & 6.73198798566141 & 0.09838312465828 \\
H & 4.71218915371565 & 5.69835745850267 & 1.30621282039813 \\
H & 5.28963032965484 & 7.35111141856240 & 1.06021366129389 \\
C & 4.72253051400367 & 5.33614709802126 & 5.28456714978637 \\
H & 5.67400840617252 & 5.84869965526188 & 5.45477672746684 \\
H & 4.87917313625656 & 4.57732391456145 & 4.51545177869922 \\
H & 4.42816813254258 & 4.83203494747509 & 6.21011941150763 \\
N & 1.70630059405088 & 7.25436873188991 & 3.81697261424949 \\
N & 3.69474337217835 & 6.24469180936016 & 4.84059637271186 \\
N & 3.47170806414237 & 7.15048614099397 & 2.10902448967889 \\
P & 2.91366252338828 & 6.07781205346824 & 3.33290083745972 \\
\hline \hline & & &
\end{tabular}

$\mathbf{1 \cdot}[\mathrm{F}]\left[\mathrm{C}_{5} \mathrm{~F}_{4} \mathrm{~N}\right]$

Electronic Energy $=-1792.26689883$

Total Thermal Energy $=-1791.93098996$

Enthalpy $=-1791.93004575$

Free Energy $=-1792.00381517$

$\begin{array}{cccc}\text { P } & 8.15350780196051 & 5.80826932796428 & 21.42666020852046 \\ \text { F } & 7.28686580895328 & 6.93192626685017 & 22.23826924829437 \\ & & & \text { S64 }\end{array}$


F 6.61109246470547

F 6.42253228463042

F 4.45980597300993

F 4.30385594153782

N 9.18054055153045

N 9.05745857919611

N 8.33122765047832

N 4.39916869295603

C 6.64030807507582

C 9.87495990455178

C 9.81302925081769

C 6.09221497895702

C 9.63026729831127

C 4.97725940085772

C 9.12500380289035

C 6.00022160634405

C 10.53842786230810

H 10.54903665863543

C 4.89691067391128

C 8.98611396548910

H 8.43263101279324

H 8.50201328599731

H 10.00114397620187

C 10.43320039145963

H 10.38556260456251

C 11.17248880196244

H 11.69661331706357

C 11.12244615551459

H 11.61238233238821

C 10.46254585283118

H 10.87154689146506

C 9.42541099063075

H 9.03378507781810

C 7.69110763531862

H 8.44774076970205

H 7.06854931019582

H 7.07025720188028

C 10.77327885288045

H 11.42245420385533

C 10.25700055787813

H 10.49687555049258
4.74517551152693

4.56858657017962

3.16402409940463

2.96988535345161

4.66624544492388

5.49772274357156

6.86222781014800

3.07654327925010

4.74114651916565

3.82018563420654

4.32125874112818

4.34572298996846

5.11294208588138

3.52409034714350

6.38881792283166

4.25879441014226

2.63956402080082

2.22082597136787

3.43065720938716

6.16368856850489

5.56458433184855

7.12684360899605

6.32135815916579

3.65716806587096

4.03509642947477

1.97398489698399

1.04772104729232

2.47821685748490

1.94884790637174

4.49811862524282

3.51642148475128

7.04982448646235

8.03752890181002

8.15326799538082

8.93139809007427

8.40915582314048

8.12131786097942

5.16839612503605

4.69069687549892

6.42353455283096

6.93061704750423
18.96516563149255

23.69197736163904

18.94053586494672

23.42813390266966

20.56822625833105

22.81538613396727

20.13280970426818

21.18545677424733

21.33543574593696

21.43227324301422

22.73548681400642

20.12376077333319

19.33049633513850

20.10510824443638

19.07746038489472

22.46716829744317

21.16497697264456

20.16884956651114

22.33717202523795

24.10454263544102

24.83137795295530

24.00662265144017

24.47486744684871

23.77596959847107

24.78852444337569

22.21214491124023

22.01288389681662

23.49857650328943

24.30634199689105

18.41668642189230

18.60346767014234

17.90458551098973

17.70182371346129

19.93488053271076

19.80857306794254

20.78094090757349

19.03592181441565

17.23472182761041

16.51144900641938

16.97702790571106

16.05091709338739

1- $[\mathrm{H}]\left[\mathrm{C}_{5} \mathrm{~F}_{4} \mathrm{~N}\right]$

Electronic Energy = -1692.95071632

Total Thermal Energy = -1692.60910378

Enthalpy $=-1692.60815957$

Free Energy $=-1692.68056675$

\begin{tabular}{llll}
\hline P & 8.21467649178512 & 5.81194895743264 & 21.44629017876001 \\
H & 7.54125918004689 & 6.84230187460637 & 22.16551563522761 \\
F & 6.89800239905504 & 4.30469911946017 & 19.01281494427306 \\
F & 6.16943975478982 & 5.08734047903847 & 23.62198914101811
\end{tabular}


F 4.67600915784190

F 3.98412527248634

N 9.31810828757169

N 9.04349278393589

N 8.30631653712495

N 4.35026788636616

C 6.67815532698892

C 9.99401986092196

C 9.84179401627194

C 6.23989572779159

C 9.69230769988203

C 5.07993554961164

C 9.07916321372193

C 5.87188689824020

C 10.71800428133210

H 10.80176129246715

C 4.73363191772080

C 8.88593484555858

H 8.39880181398788

H 8.26898180573381

H 9.86094011099683

C 10.43781766207812

H 10.32529192751985

C 11.32024723715032

H 11.88923867750288

C 11.18811986657505

H 11.66004359879739

C 10.54616165793926

H 11.04648712326827

C 9.28802768568439

H 8.80471176038631

C 7.61312021991009

H 8.31110590113439

H 7.12600830583903

H 6.84573768607460

C 10.76395988391591

H 11.42835152061761

C 10.13917955621642

H 10.30809761715827
2.83402333216373

3.56428742072889

4.73001755531312

5.39511422425988

6.83284171610484

3.20310649472289

4.75572174428799

3.85339108351119

4.25877316730860

4.15723146538819

5.15430481511801

3.39695604310615

6.37932362492790

4.54296884869657

2.71529530876174

2.35704490084381

3.76179689350478

6.04429438802728

5.39095190660587

6.93378408048845

6.34656022507125

3.55491297770475

3.87220287067978

2.00566461303641

1.11349215633241

2.42235853132243

1.86145906725925

4.57555535082747

3.64232749073381

7.01015504147593

7.95195510665815

8.09338127644710

8.90193224648070

8.32382154807593

8.05753588308083

5.21740449761897

4.76127066040388

6.41337458991474

6.89505642246831
19.03179824097302

23.41268666057639

20.59207331749736

22.87201751522279

20.09659178927083

21.22364615468015

21.33058930606659

21.43488732465660

22.76863308917276

20.15823670017118

19.32290405484217

20.16010682922045

19.02928606740501

22.43950433829664

21.13595223255342

20.12067288456757

22.33815342861431

24.15120549891567

24.87914581993617

24.03430564189594

24.54232849111563

23.79633009526277

24.82521460066410

22.17385729530715

21.94380828920291

23.48519018934903

24.28236628625141

18.40497265627011

18.61298672687474

17.82117015464711

17.59439791187767

19.99056428961547

19.75739873644135

20.93728145406557

19.21202873214510

17.18565442215345

16.46216386375256

16.89137397062165

15.93640504056661

\section{TS1}

Electronic Energy = -1692.86054889

Total Thermal Energy $=-1692.52342239$

Enthalpy $=-1692.52247818$

Free Energy $=-1692.59540058$

$\begin{array}{clcc}\text { P } & -0.07670969200994 & 0.00030341337562 & 0.54593031586610 \\ \text { F } & 1.68080745143824 & 2.38284385063991 & -0.27315536503079 \\ \text { F } & 4.24298963016778 & 2.24555310834771 & -1.04789785162279 \\ \text { F } & 4.24070658795624 & -2.24822652862749 & -1.04881618522569 \\ \text { F } & 1.67836327438153 & -2.38320027869709 & -0.27416440581792 \\ \text { N } & -1.35002092871316 & 0.00064002401436 & -0.55726337797197\end{array}$


N -0.48897201269299

$\mathrm{N}-0.48980476897768$

N 4.31266020465413

C -1.50259067353342

C -1.98815595193852

H -1.59315871874416

C -3.00313348920508

H -3.39416931604751

C -3.50407683777037

H -4.28636126126327

C -2.99691197206927

H -3.36814846746577

C -2.00436423707768

C -2.00391757896534

C -2.99538919324309

H -3.36593362642825

C -3.50216679031325

H -4.28354478735394

C -3.00187079441222

H -3.39257771138242

C -1.98786427544640

C -1.50266489998046

C 0.16811319341823

H 0.73531165841632

H 0.85582252677904

C 0.16935385311568

H -0.57690007355626

H 0.73545384643738

H 0.85820252574764

C 1.62989407606410

C 2.32310748411263

C 3.61615578380417

C 3.61726448007060

C 2.32427376985616

H -0.57855528758400

$\mathrm{H}-1.59323884393508$

H 1.54978184368963
1.44229403355800

$-1.44152596024364$

$-0.00139590959636$

2.07096361885456

3.34848260839631

3.99012310094219

3.80021462703261

4.80082453015395

2.99430711856730

3.36316390902594

1.71197075995770

1.09426597985473

1.25811518890850

$-1.25710458677288$

$-1.71106895256691$

$-1.09327580636791$

$-2.99371343123407$

$-3.36271378343573$

$-3.79979974370265$

$-4.80064949081466$

$-3.34801431693434$

$-2.07020810040560$

$-2.15096062197957$

$-3.00206832714645$

$-1.47983656888890$

2.15145370212948

2.49440970834715

3.00331466842933

1.48057802926788

$-0.00024920341836$

$-1.18700384897152$

$-1.10874846634344$

1.10659292486924

1.18599088753872

$-2.49509425789032$

$-3.98986689161673$

$-0.00043071655650$
1.23625326281968

1.23620349812891

$-0.90584728981042$

0.48387545497702

0.66915315813859

1.44560163822739

$-0.17148826032187$

$-0.03664426158450$

$-1.17712870817540$

$-1.82798803407754$

$-1.37618429178981$

$-2.18179821706742$

$-0.53788162904311$

$-0.53899271384708$

$-1.37851792885861$

$-2.18438943313103$

$-1.18050857483291$

$-1.83238133696955$

$-0.17467963520071$

$-0.04066294999865$

0.66709145702983

0.48281518215736

2.31686743578286

1.93716810900775

2.82773388057063

2.31685614927901

3.03636758582348

1.93721839030930

2.82650386306624

0.15807374555335

$-0.32618531243395$

$-0.74156696049207$

$-0.74114227212916$

$-0.32576326837583$

3.03537957632869

1.44355192521917

1.45009363552343

\section{TS2}

Electronic Energy = -1692.88294884

Total Thermal Energy $=-1692.54590272$

Enthalpy $=-1692.54495852$

Free Energy $=-1692.62052189$

$\begin{array}{llcl}\mathrm{P} & -0.07248107892117 & 0.47970226333680 & 1.03536402120699 \\ \mathrm{~N} & 0.67522287644114 & -0.82749497191715 & 0.31200565797535 \\ \mathrm{~N} & 0.99054013417152 & 0.58718634550595 & 2.28386127668799 \\ \mathrm{~N} & -0.14019235579312 & 1.39162824064617 & -0.33168518745529 \\ \mathrm{C} & 2.00523897902997 & -0.38106419044908 & 2.17587626652668 \\ \mathrm{C} & 3.06454029545709 & -0.57086123689527 & 3.03848034143003 \\ \mathrm{H} & 3.21481005454193 & 0.08106837973594 & 3.88940665990557 \\ \mathrm{C} & 3.93878106980704 & -1.62426734112931 & 2.78248867335705\end{array}$




\begin{tabular}{llll} 
H & 4.77580448238568 & -1.79149767270088 & 3.44859623679484 \\
C & 3.74677315125989 & -2.45700285837884 & 1.69290605454607 \\
H & 4.43379345817294 & -3.27302728540186 & 1.50883730267606 \\
C & 2.67074858485356 & -2.26644605519065 & 0.82854618350695 \\
H & 2.51722694125897 & -2.93033087412118 & -0.01001579086816 \\
C & 1.80967785531517 & -1.22025636072897 & 1.07538550808227 \\
C & 0.71768584999870 & -0.63328038608906 & -1.10032978369667 \\
C & 1.09533800643343 & -1.53898025721576 & -2.06466529267838 \\
H & 1.37479543999585 & -2.54946855209592 & -1.80236738097900 \\
C & 1.07817379473153 & -1.13134056607547 & -3.39672964805739 \\
H & 1.37049107707347 & -1.83123056121272 & -4.16886228875394 \\
C & 0.67228447197158 & 0.14717412336572 & -3.73966270627216 \\
H & 0.65091122699338 & 0.44429396091742 & -4.78061797625082 \\
C & 0.27143891364081 & 1.05819738449049 & -2.76558923061960 \\
H & -0.05990809179322 & 2.05163570942779 & -3.03836833241924 \\
C & 0.29710513687139 & 0.65294019592052 & -1.44760330469267 \\
C & -0.91970258940688 & 2.61061637811192 & -0.46369350248287 \\
H & -0.27667678145411 & 3.45554797657117 & -0.71244851826529 \\
H & -1.42695912452842 & 2.81928392602650 & 0.47876617244089 \\
H & -1.68207454022708 & 2.48536252179929 & -1.23319811801628 \\
C & 0.81026481297158 & 1.41129216819798 & 3.46512624475720 \\
H & 0.82523867096407 & 0.78923448798996 & 4.36101066168725 \\
H & -0.15590021676555 & 1.91301904186571 & 3.40761044832419 \\
H & 1.59127126306247 & 2.16958697399898 & 3.53416084370990 \\
F & -2.24871032348205 & -2.06930125517708 & -0.28817902291188 \\
F & -3.62368714254917 & -2.07540154171020 & -2.59419321297887 \\
F & -5.42813573996402 & 1.96586018524500 & -1.76139518876420 \\
F & -4.12915603204168 & 2.19743781652202 & 0.57719893777893 \\
N & -4.52592371969921 & -0.05884764665412 & -2.15907981435037 \\
C & -3.12064897871174 & 0.06152388214989 & 0.30346681582577 \\
C & -3.95477952097128 & 1.05345496582956 & -0.14977197304593 \\
C & -4.63002525805263 & 0.96905999576482 & -1.35382953476210 \\
C & -3.73910831921646 & -1.02886483282109 & -1.76599815547792 \\
C & -3.04600710454141 & -0.99630616566724 & -0.56908965749193 \\
H & -1.41807962928405 & 0.46016368821226 & 1.49827931407101 \\
\hline & & & \\
\hline
\end{tabular}




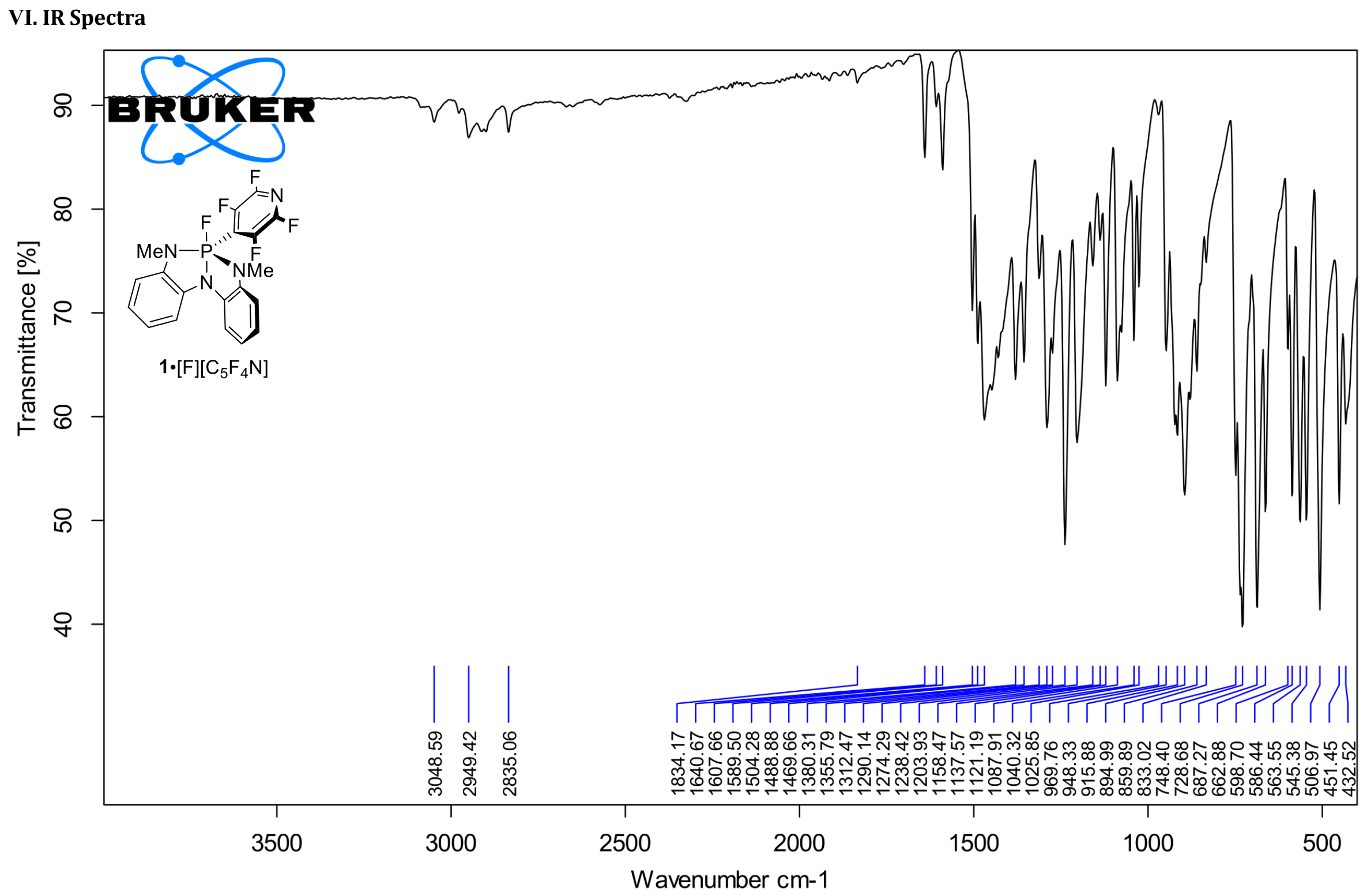




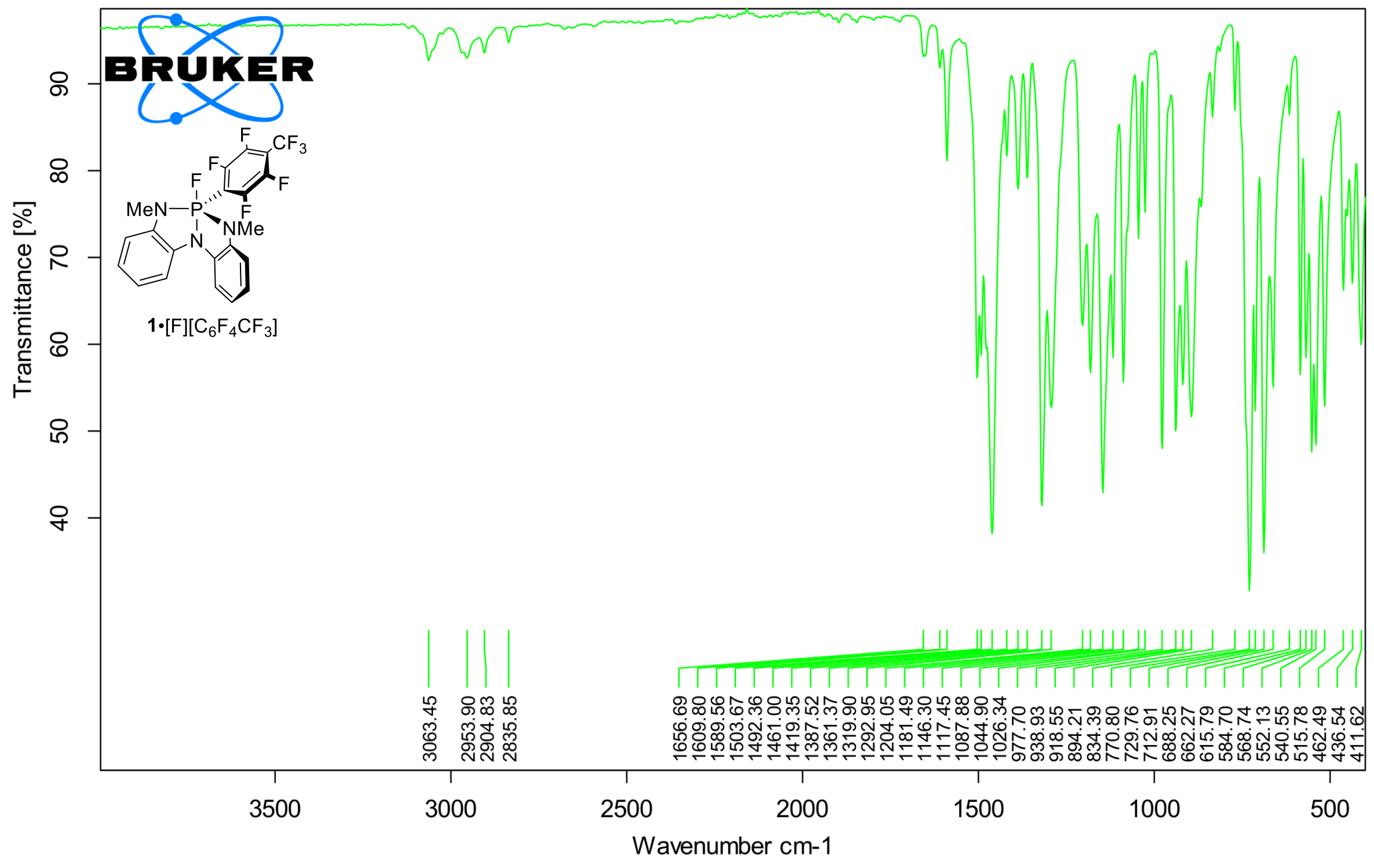




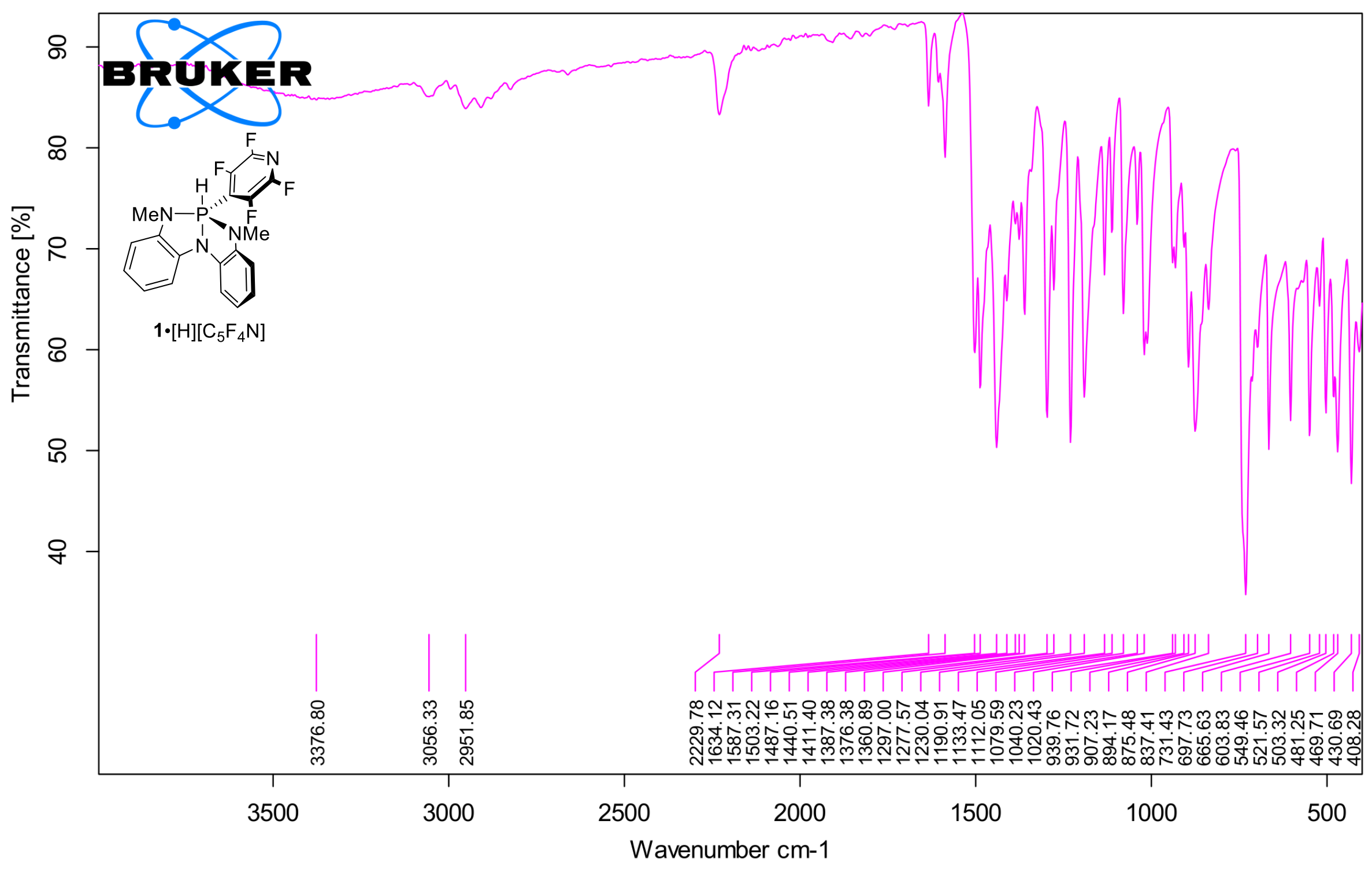




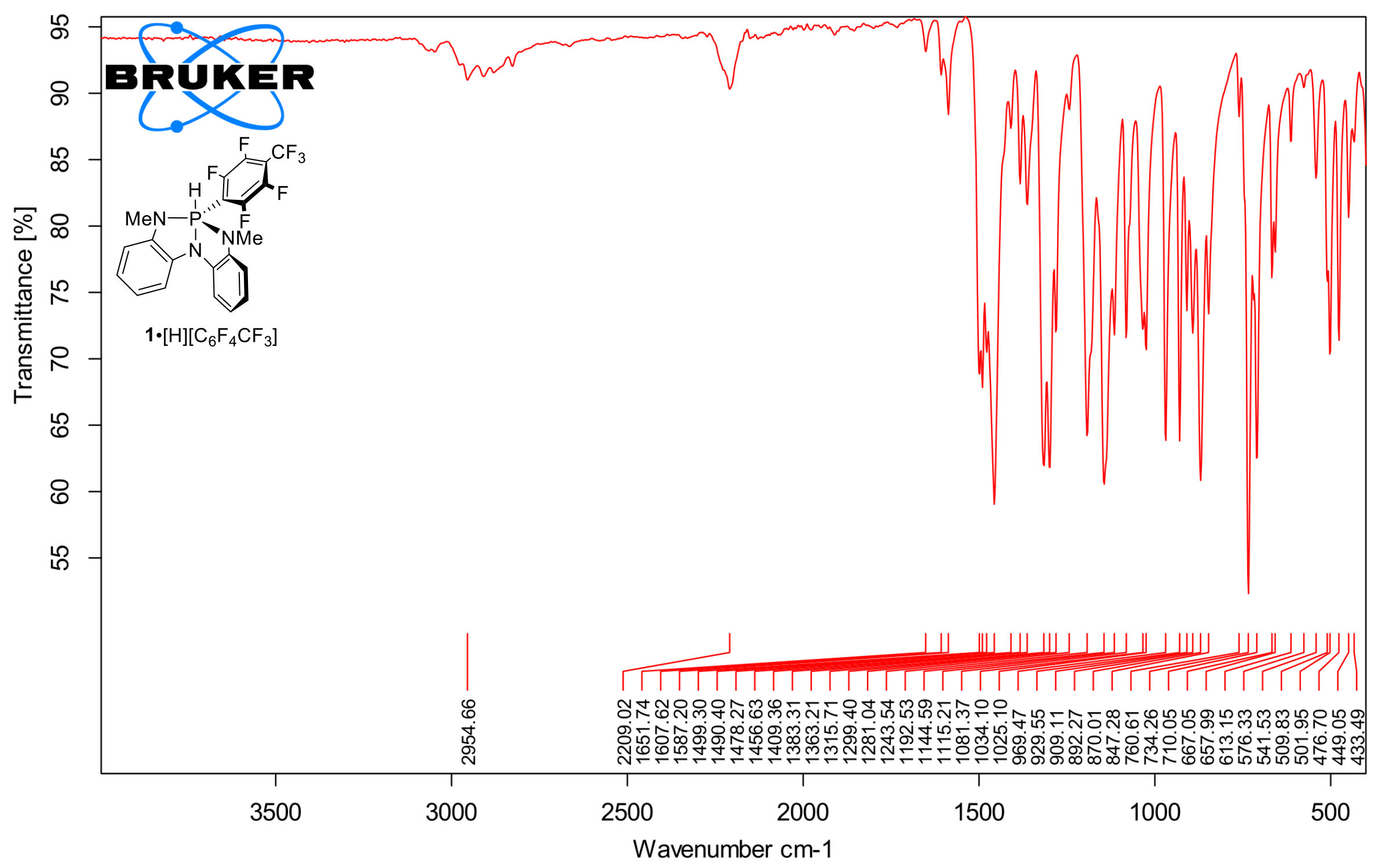


VII. Multinuclear NMR Spectra

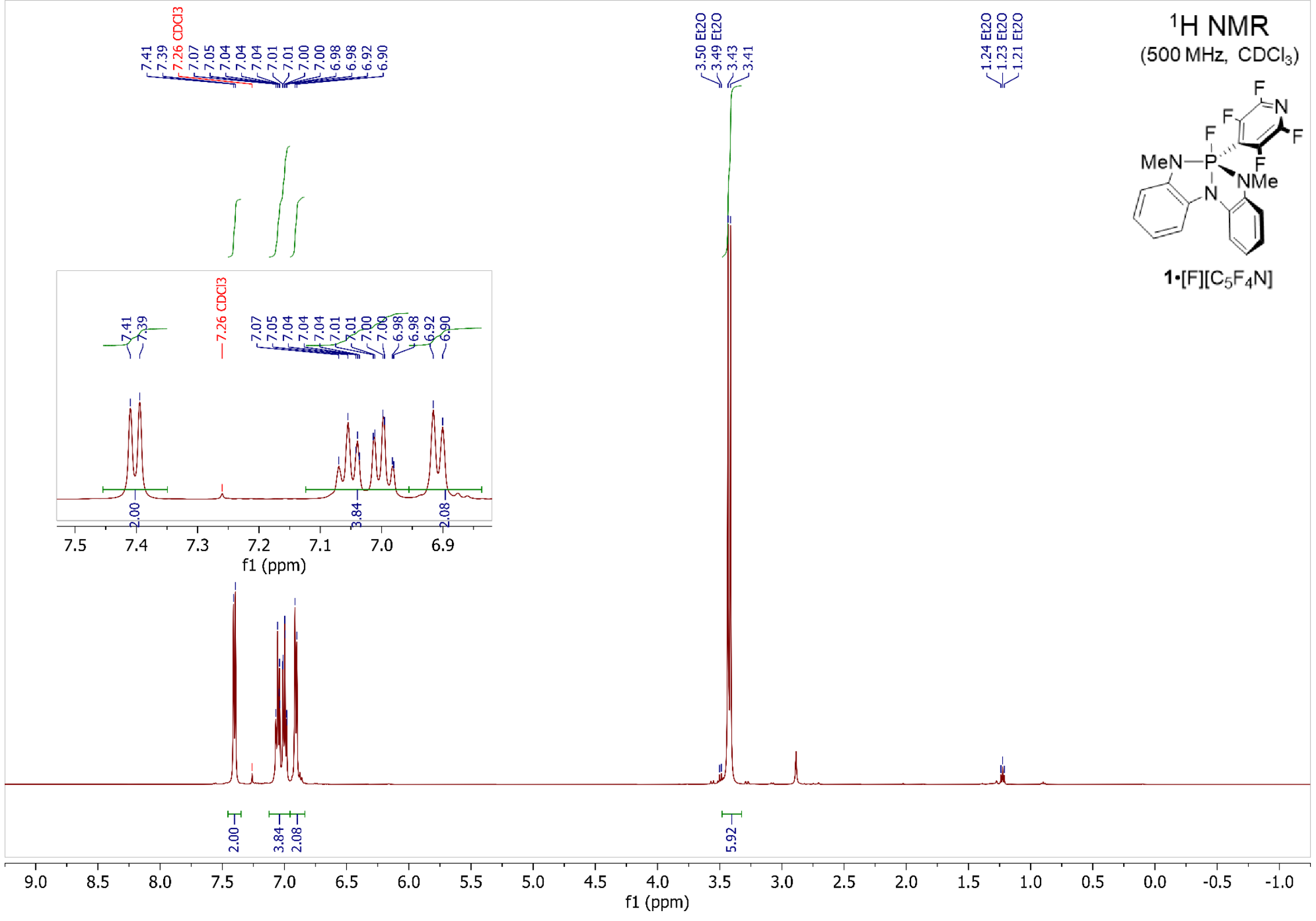




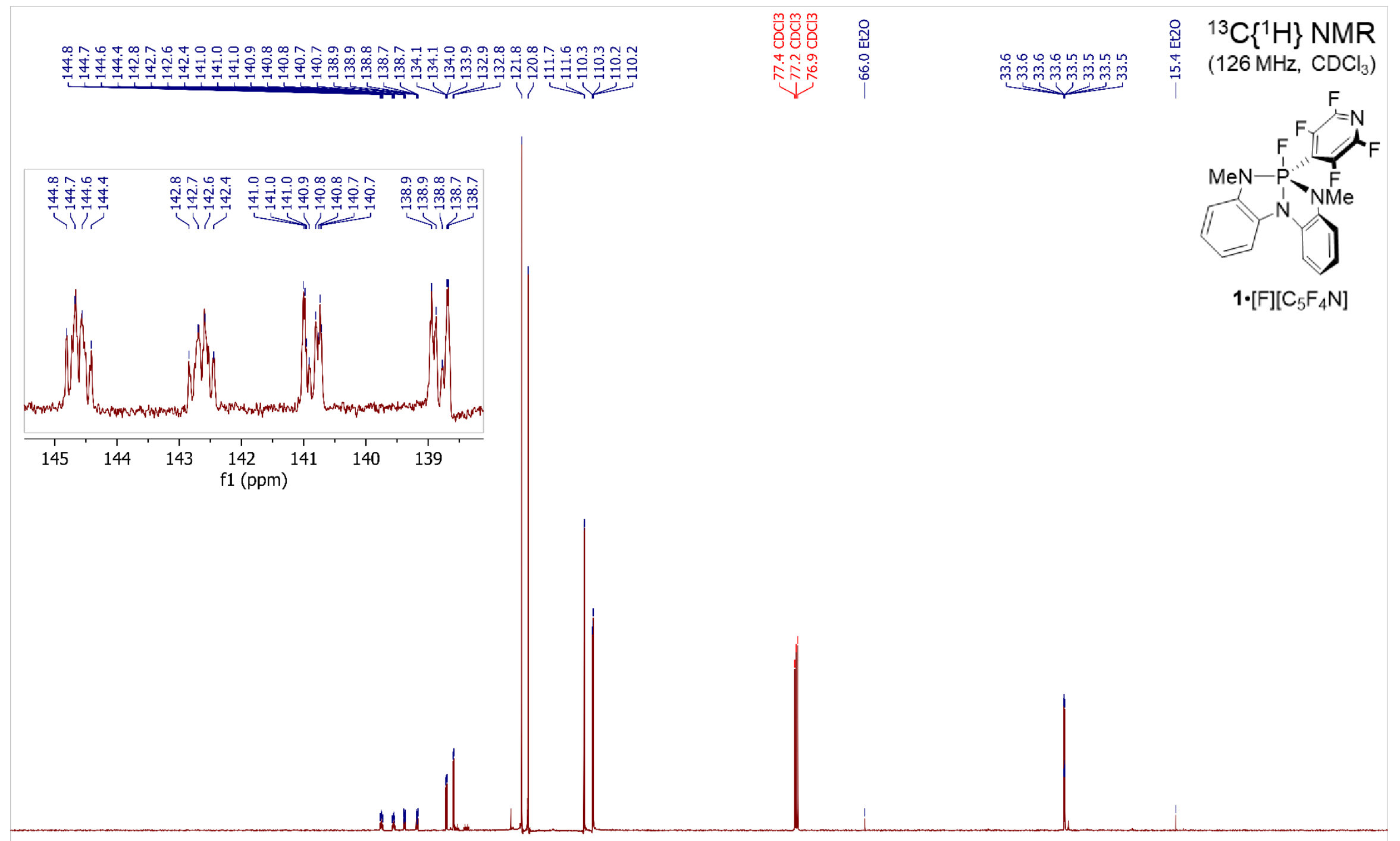

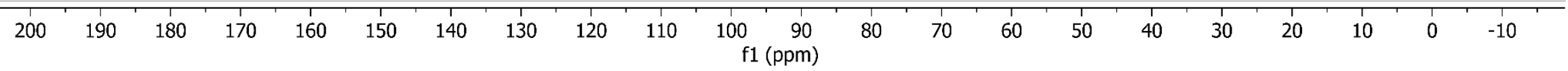




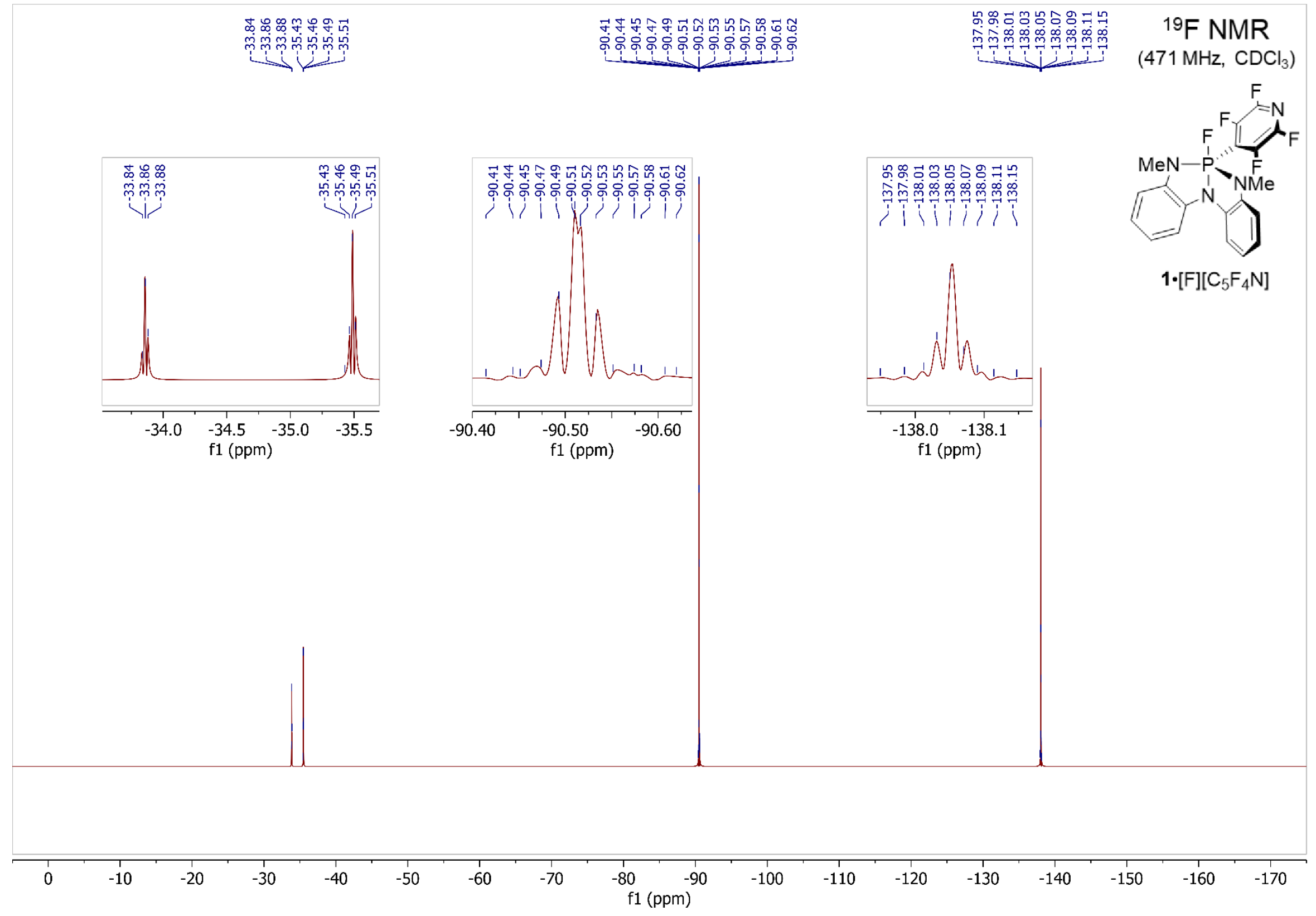




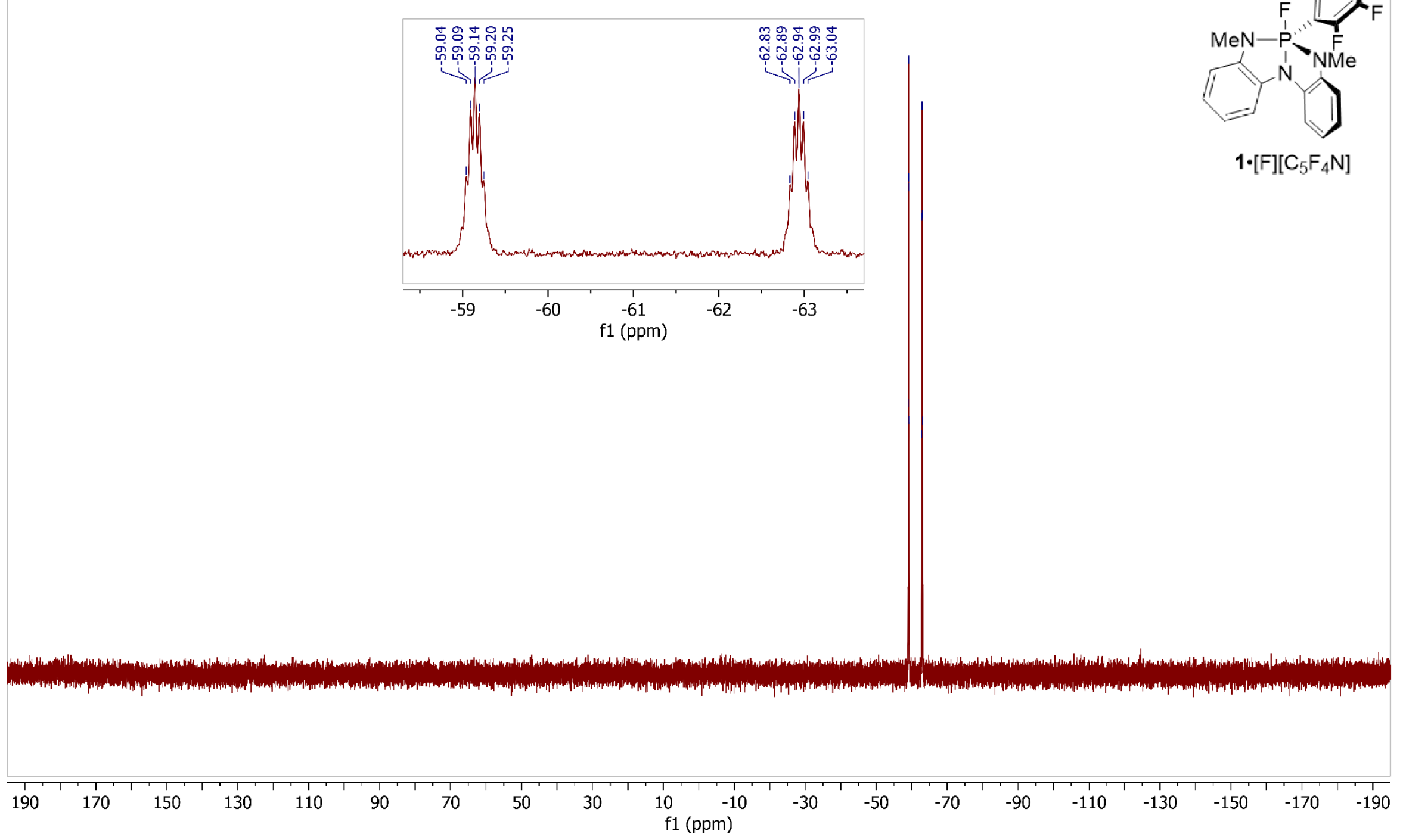

(203 MHz, $\mathrm{CDCl}_{3}$ )

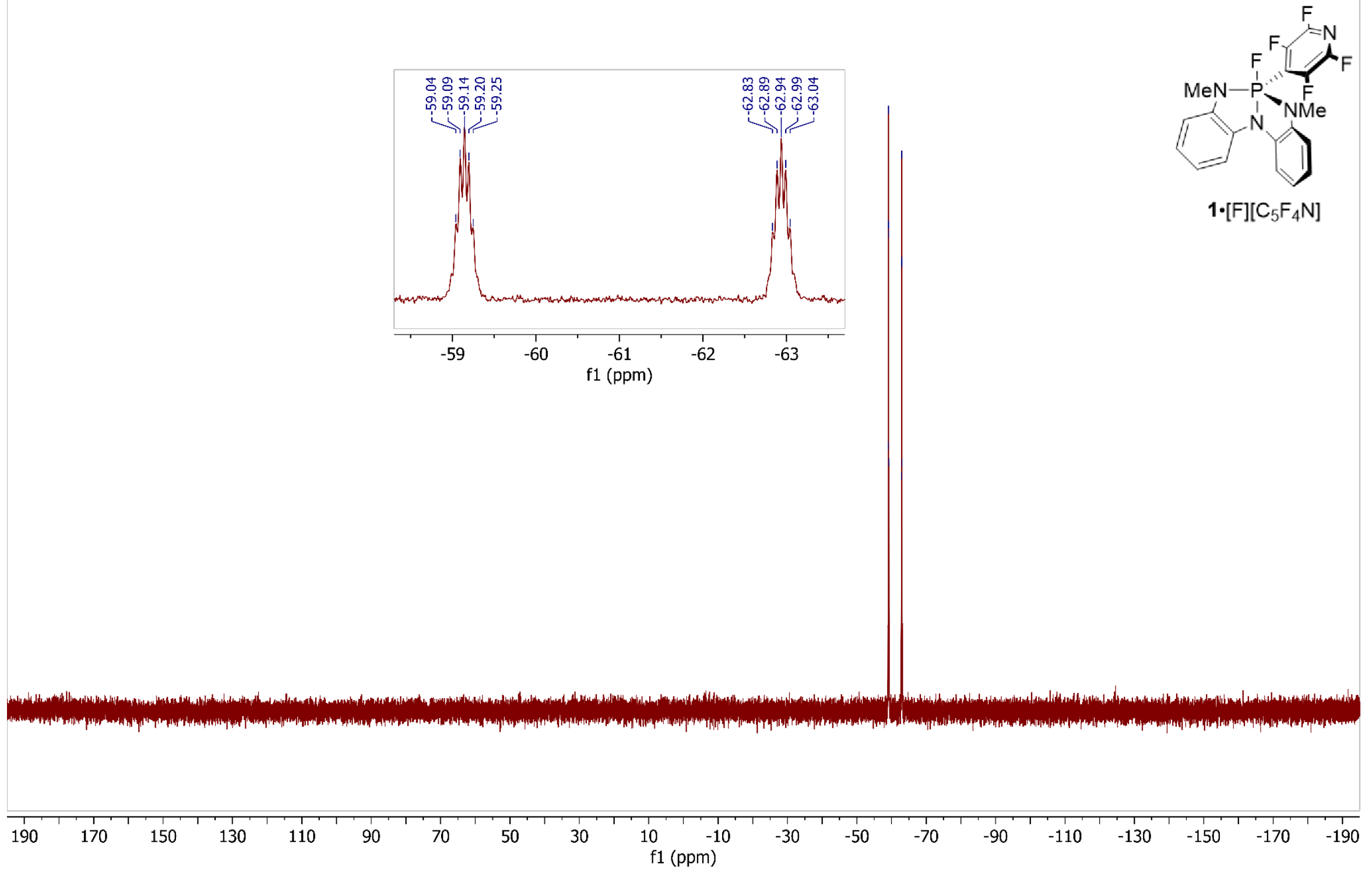

$1 \cdot[\mathrm{F}]\left[\mathrm{C}_{5} \mathrm{~F}_{4} \mathrm{~N}\right]$ 


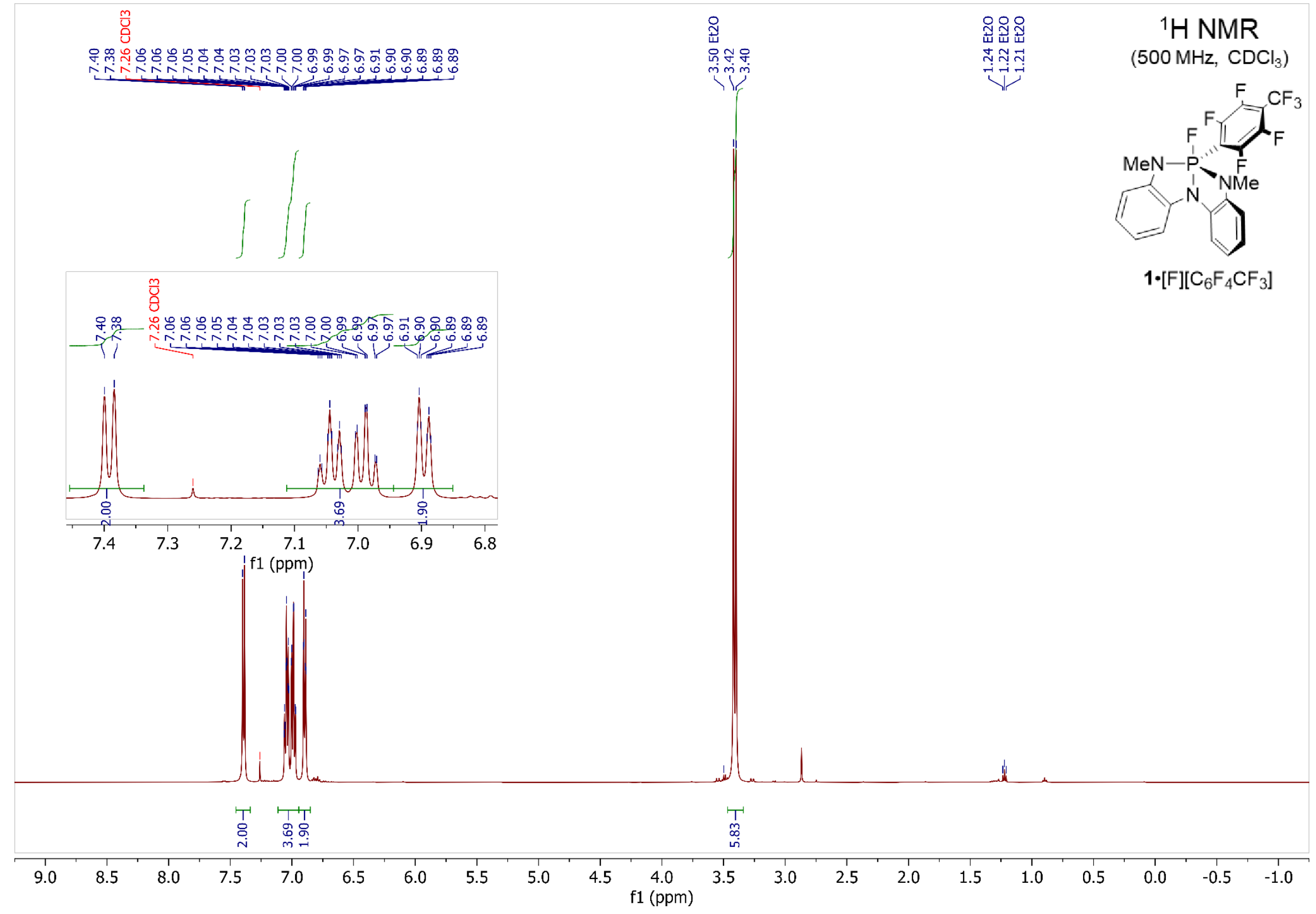




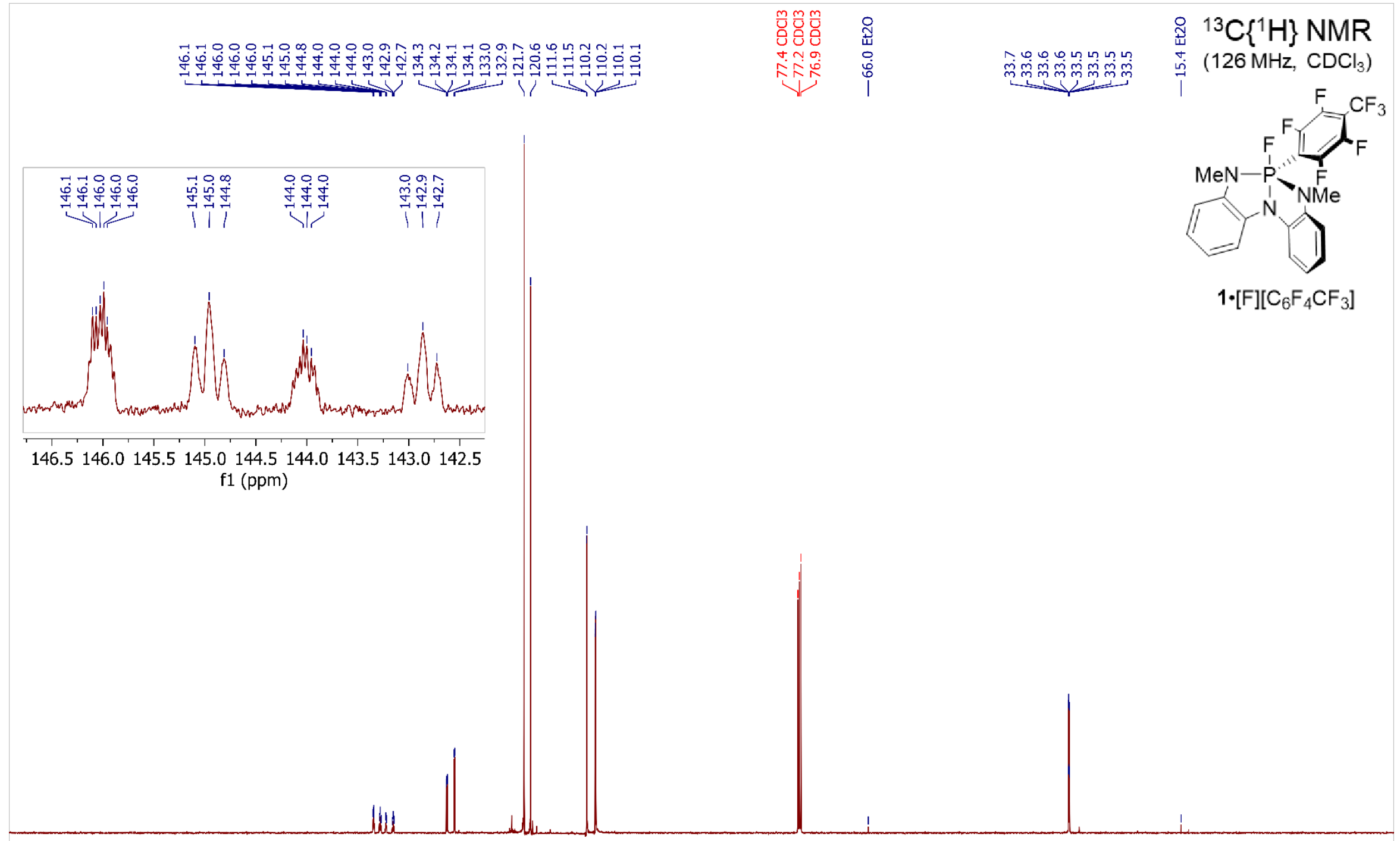

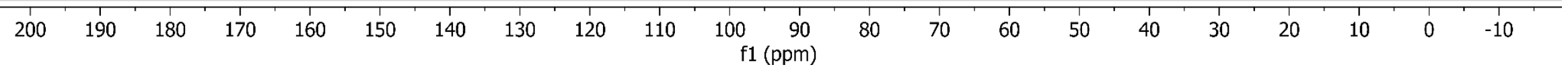




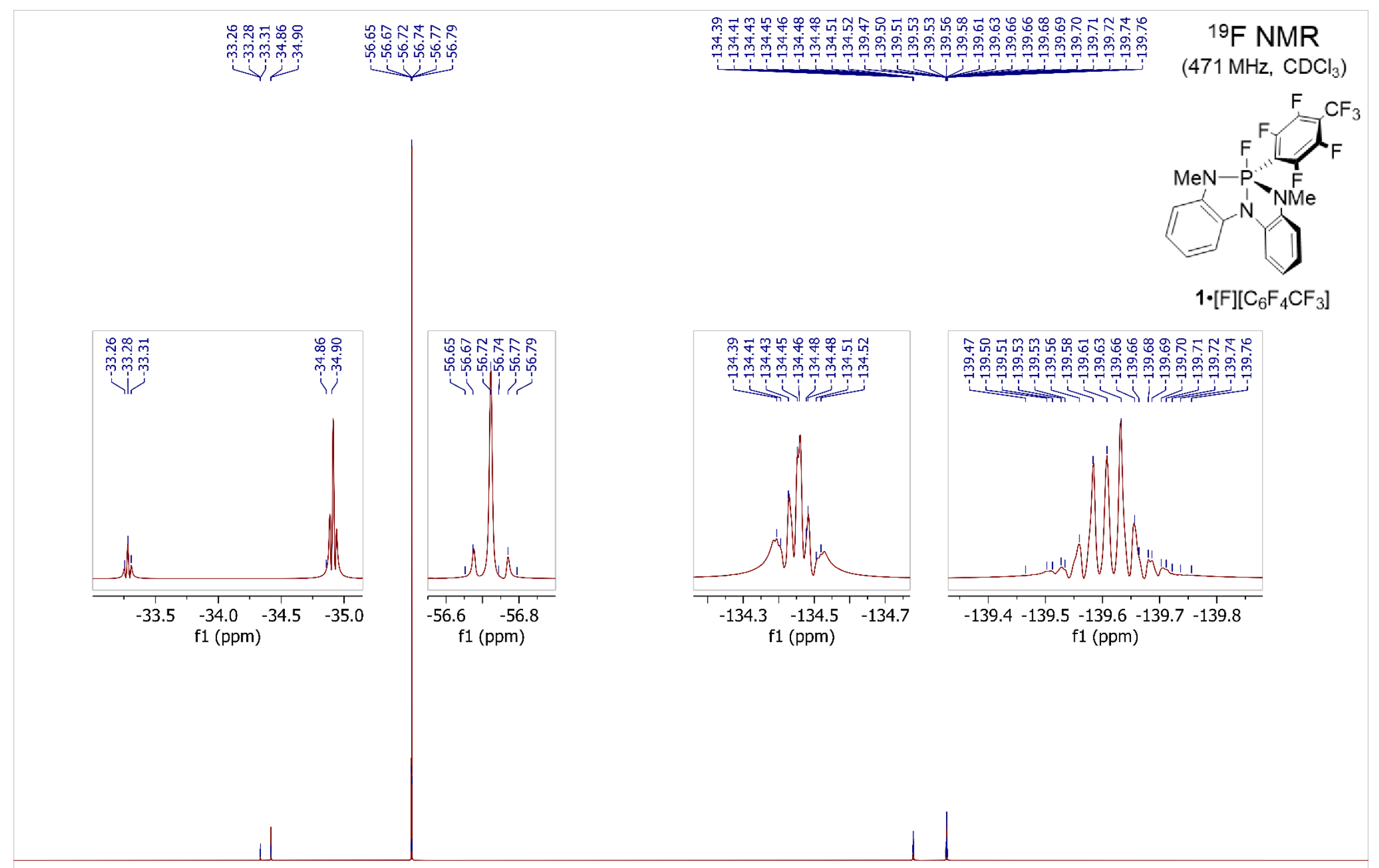

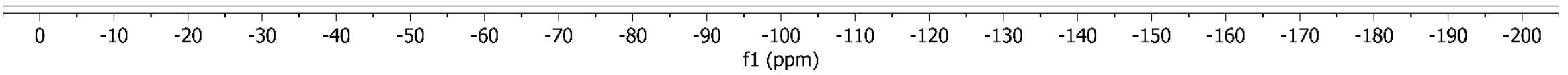




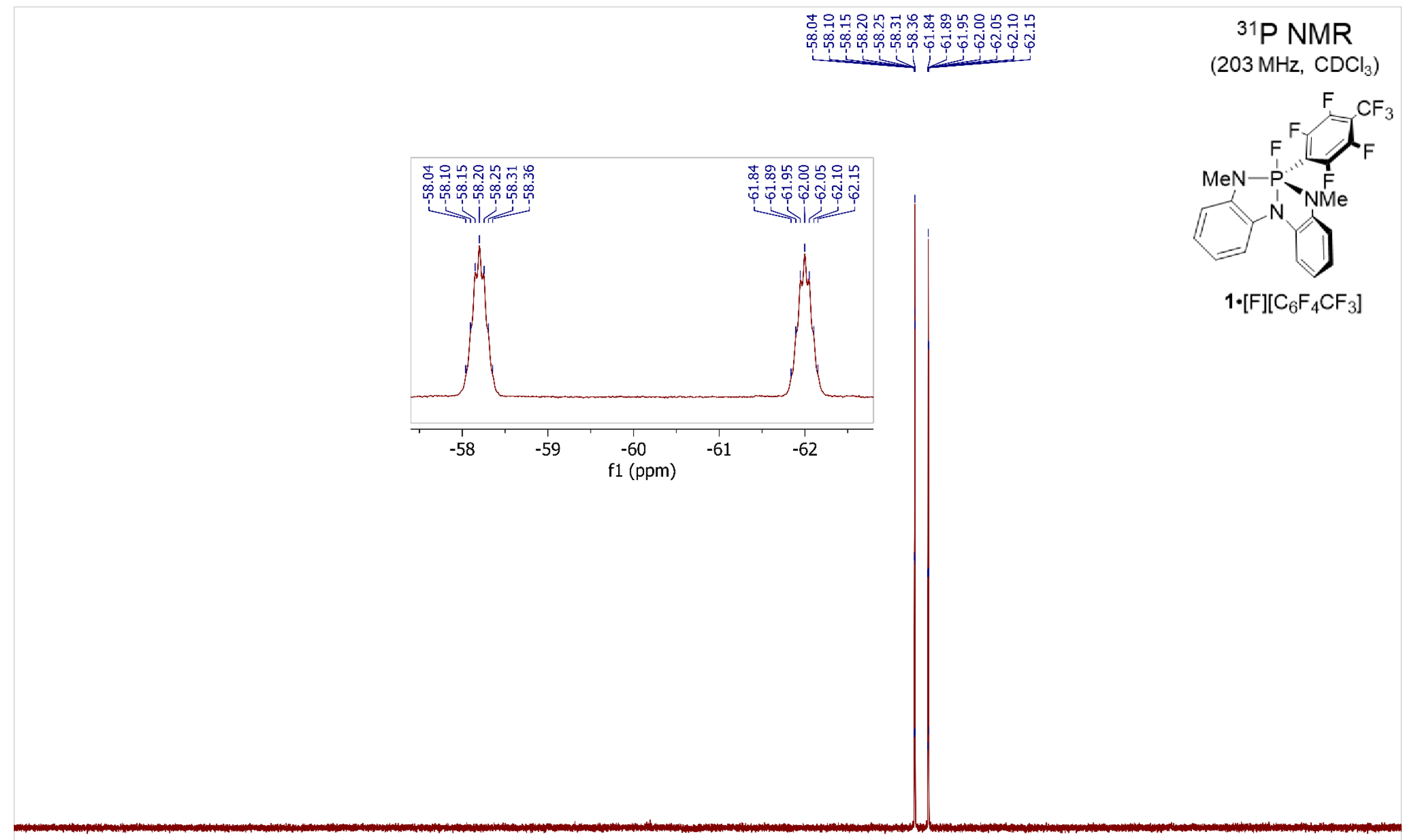

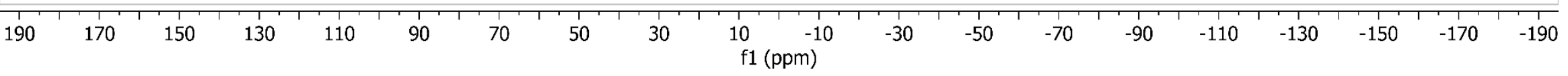




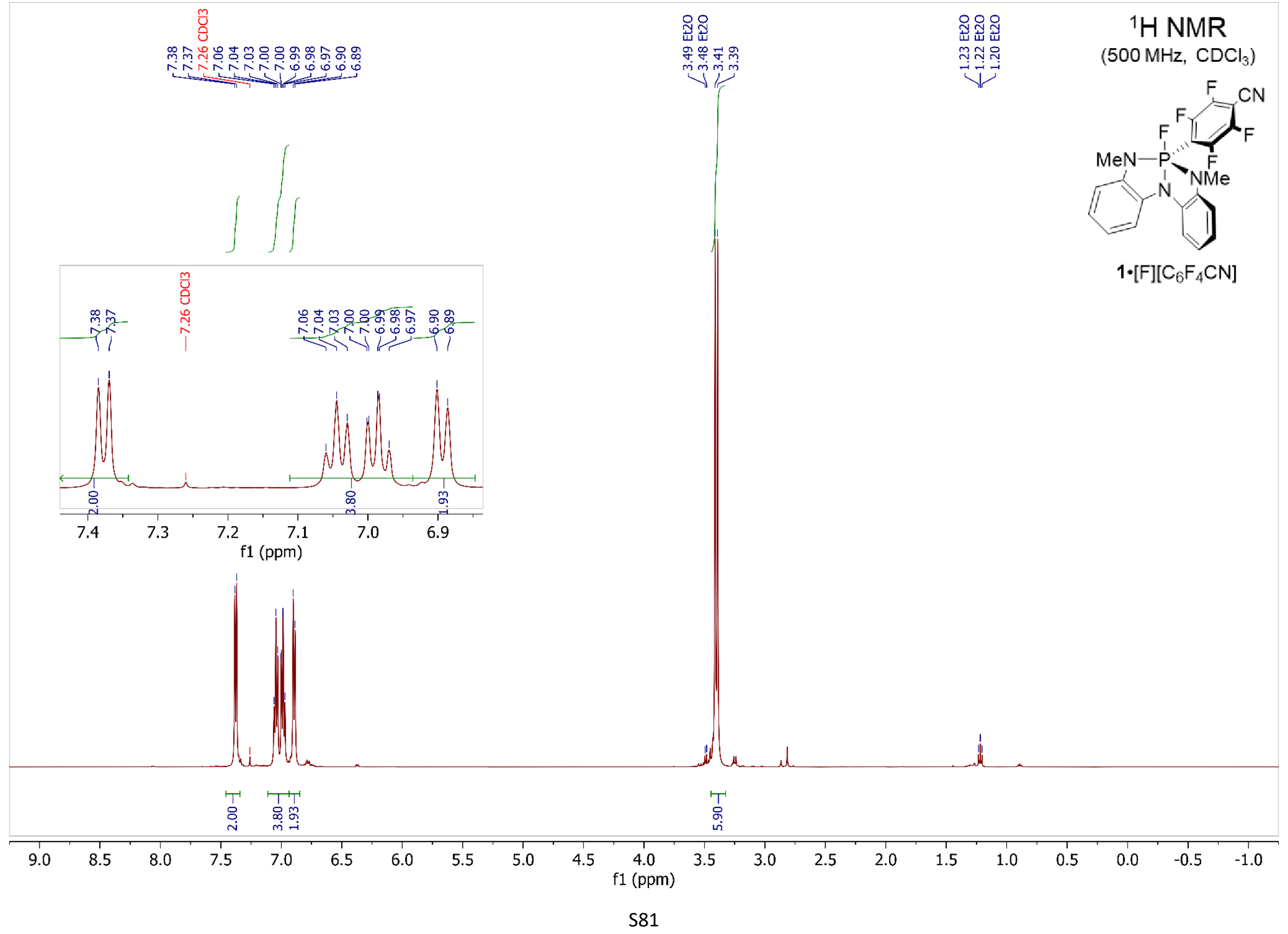




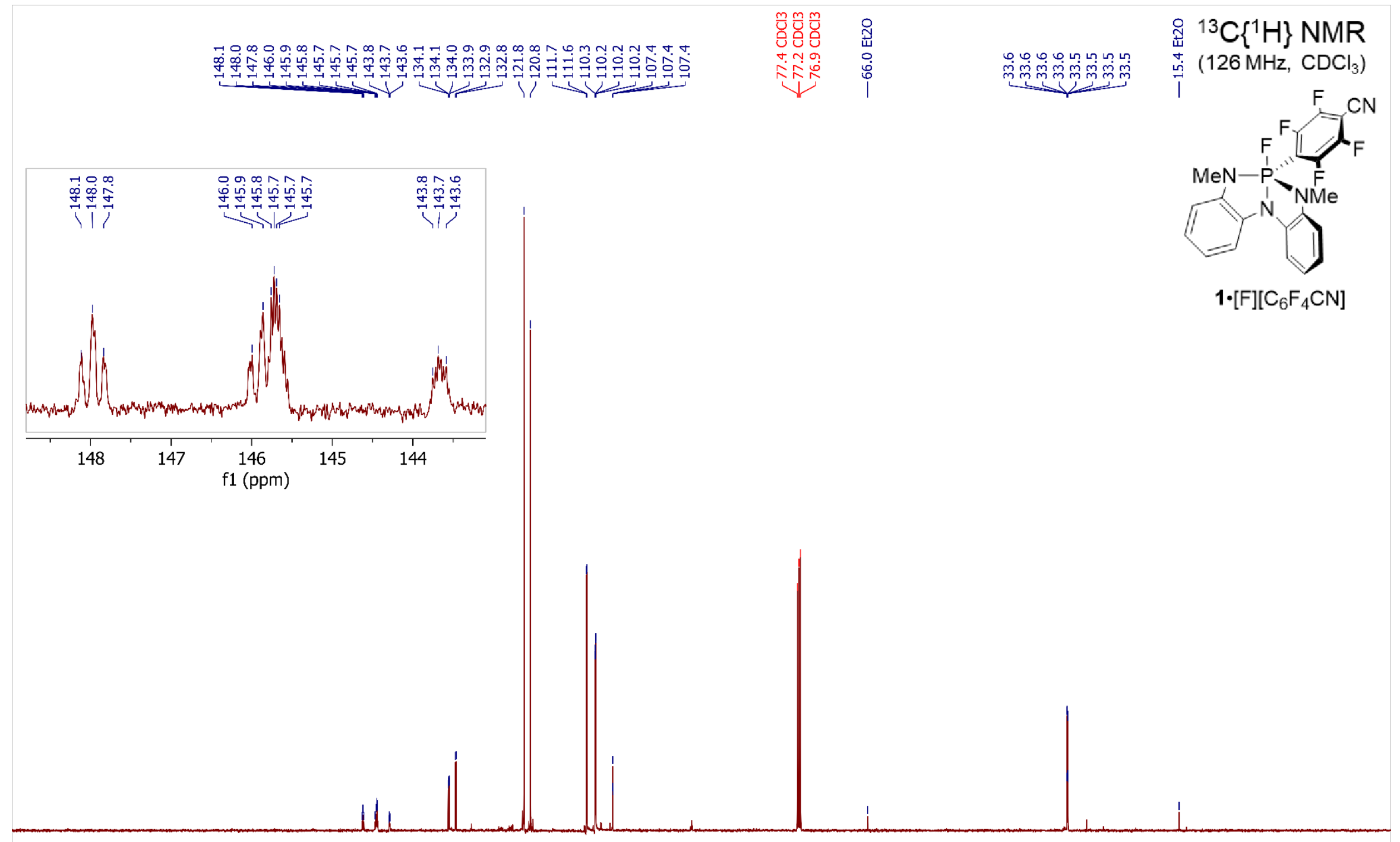

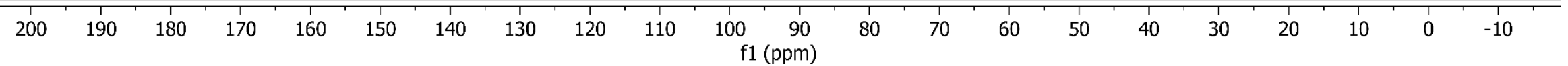




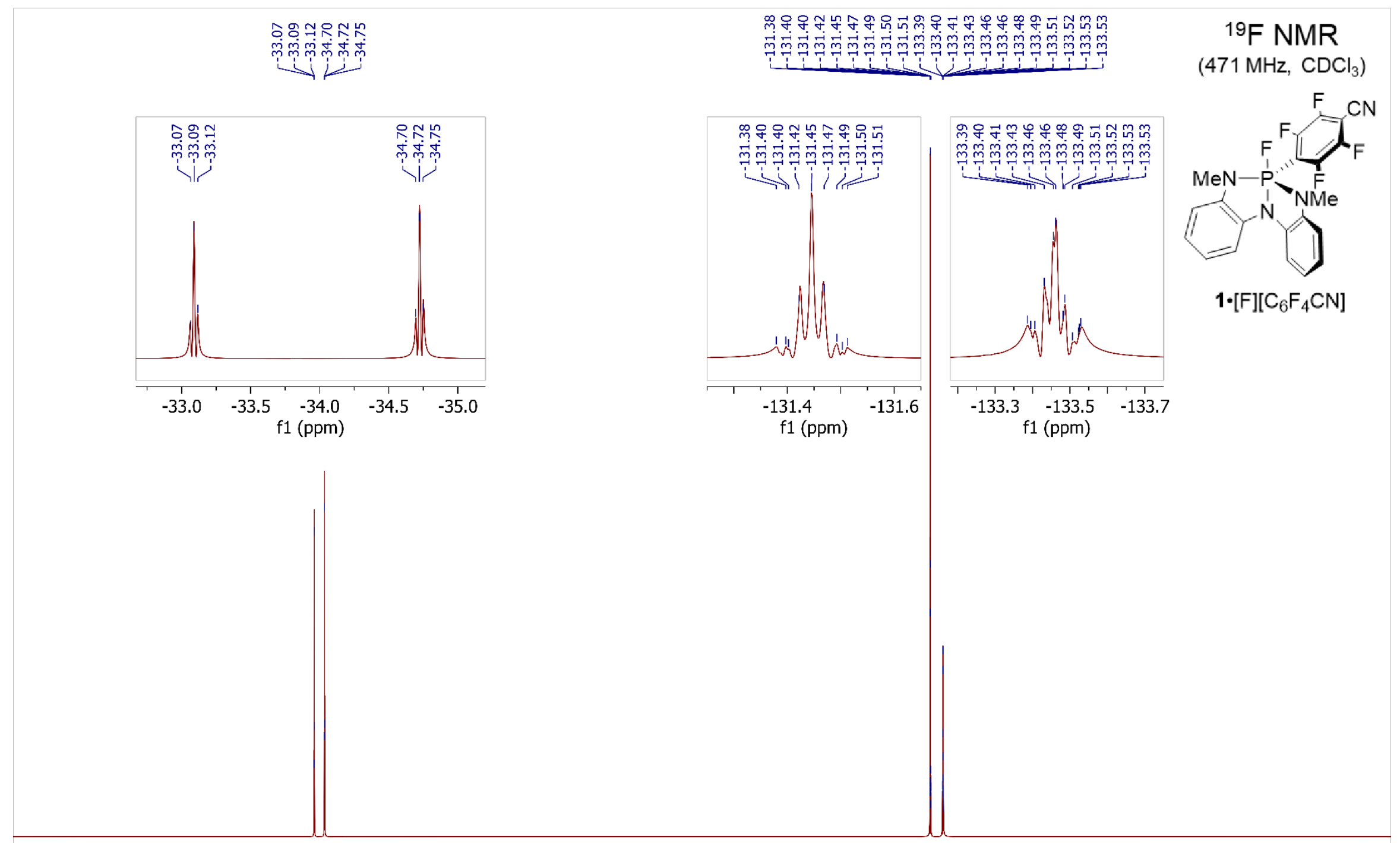

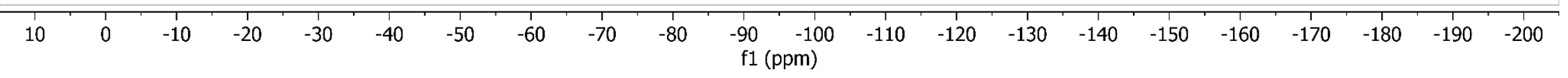




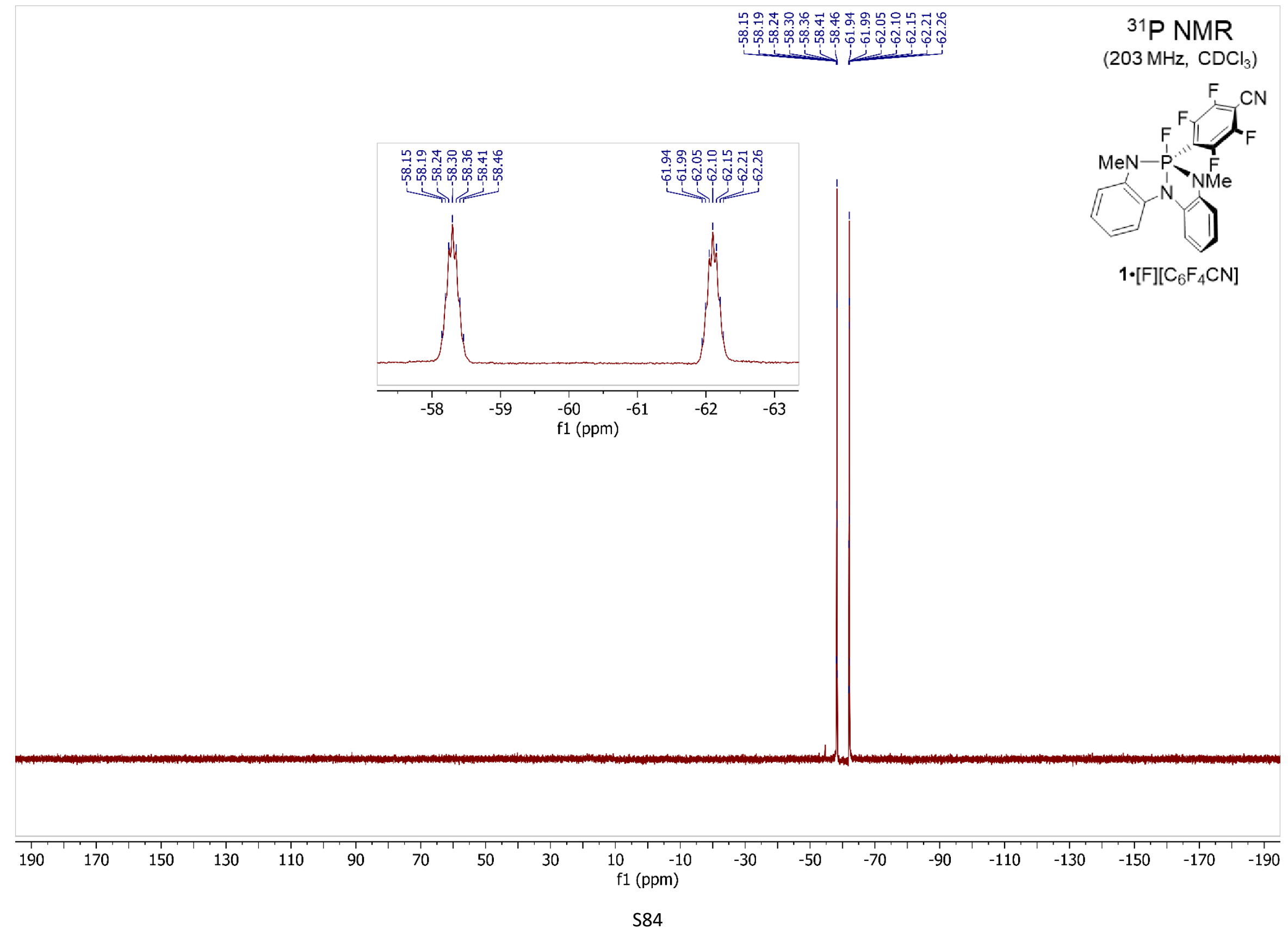




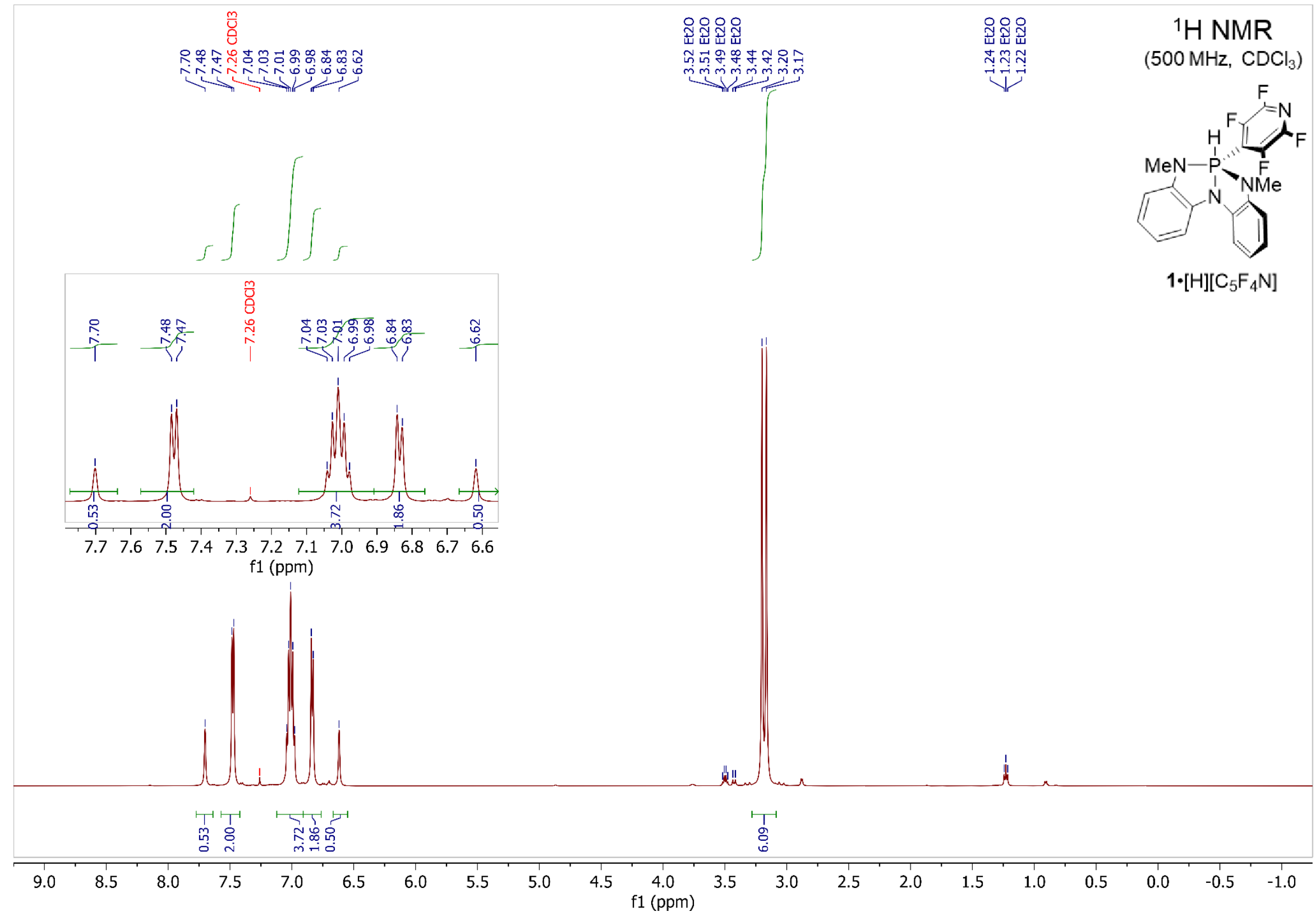



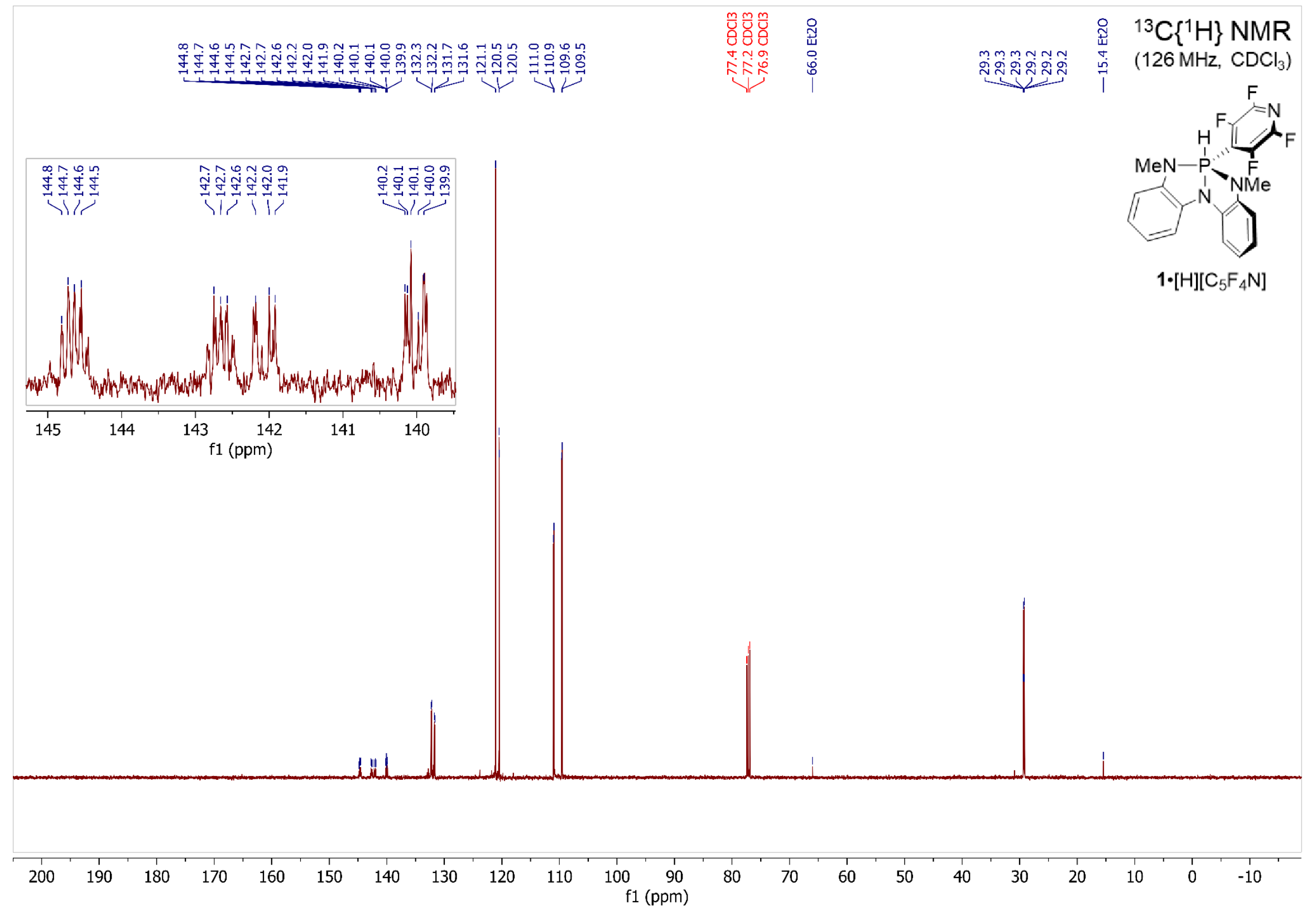


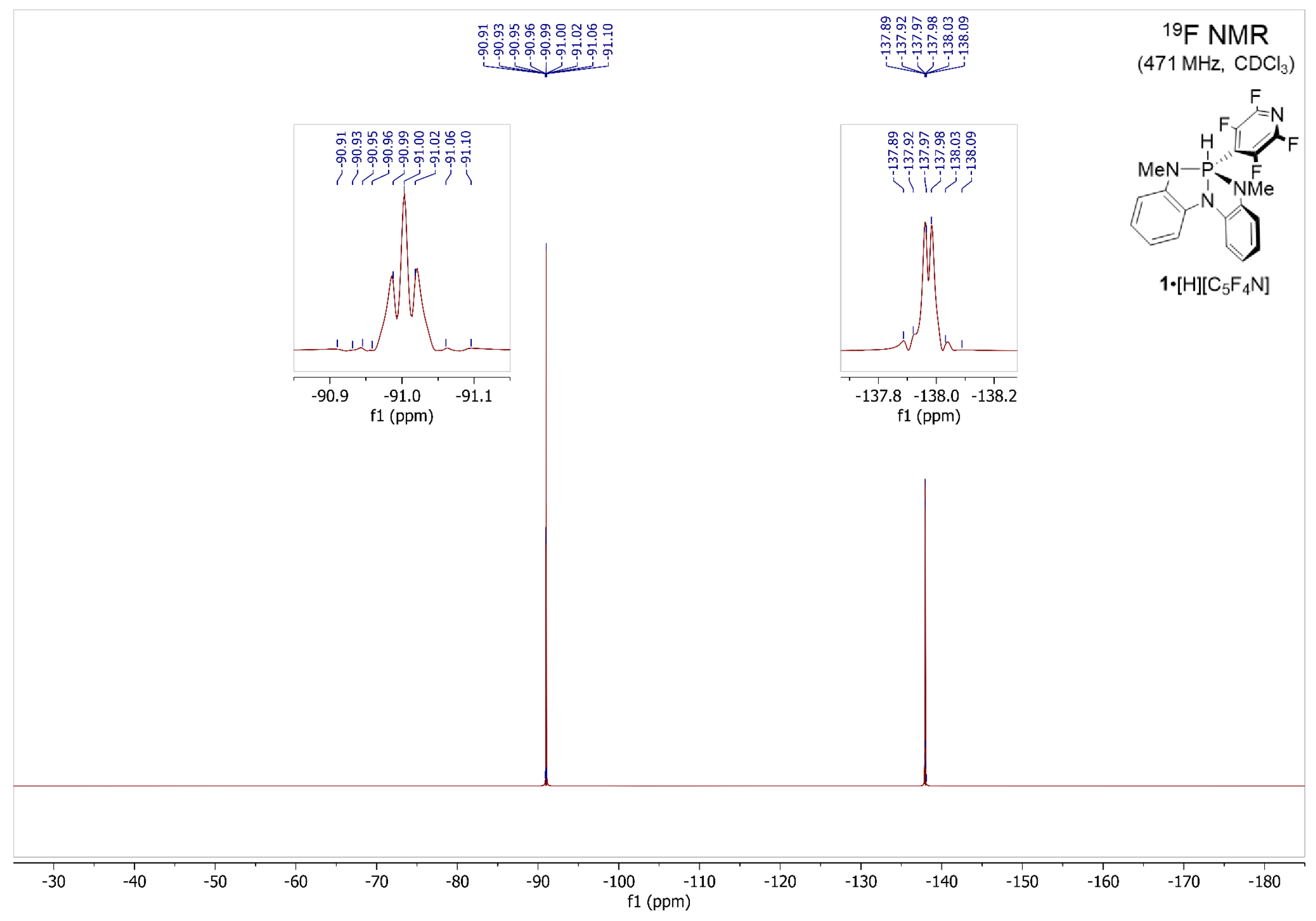



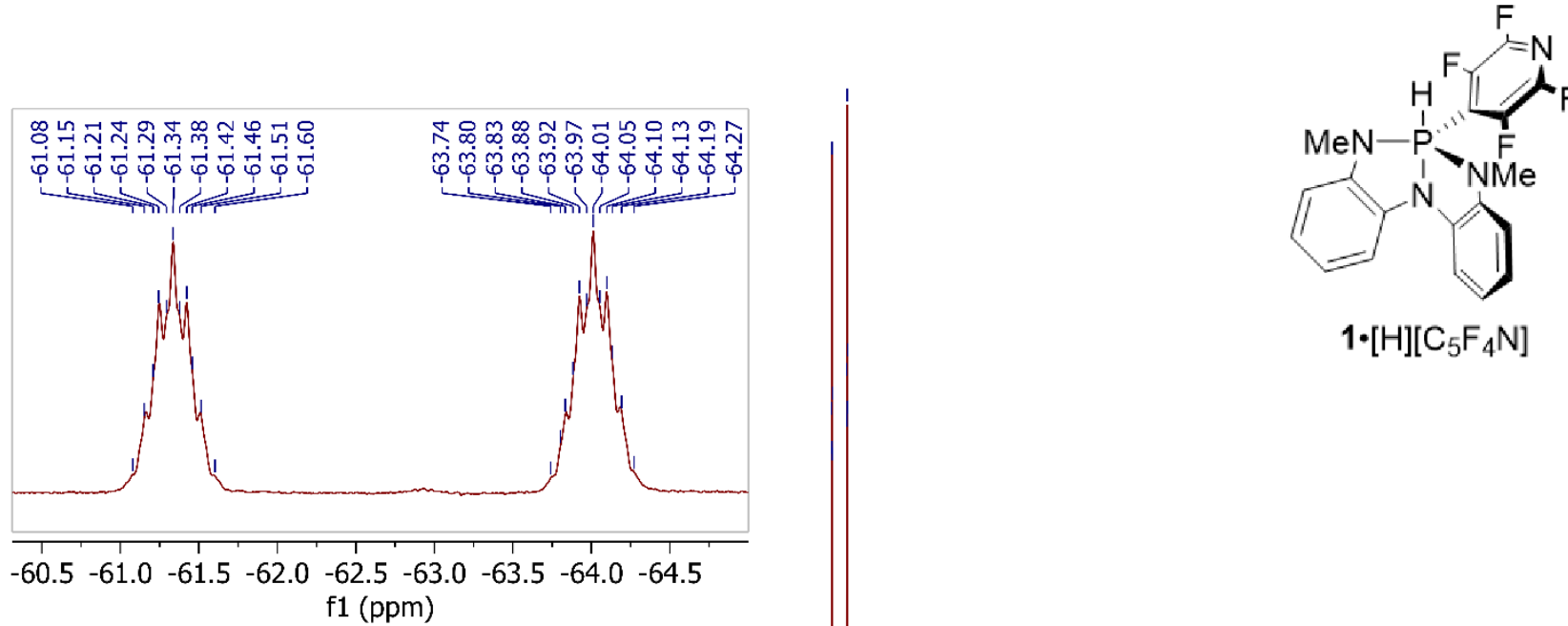

1•[H][ $\left.\mathrm{C}_{5} \mathrm{~F}_{4} \mathrm{~N}\right]$ 


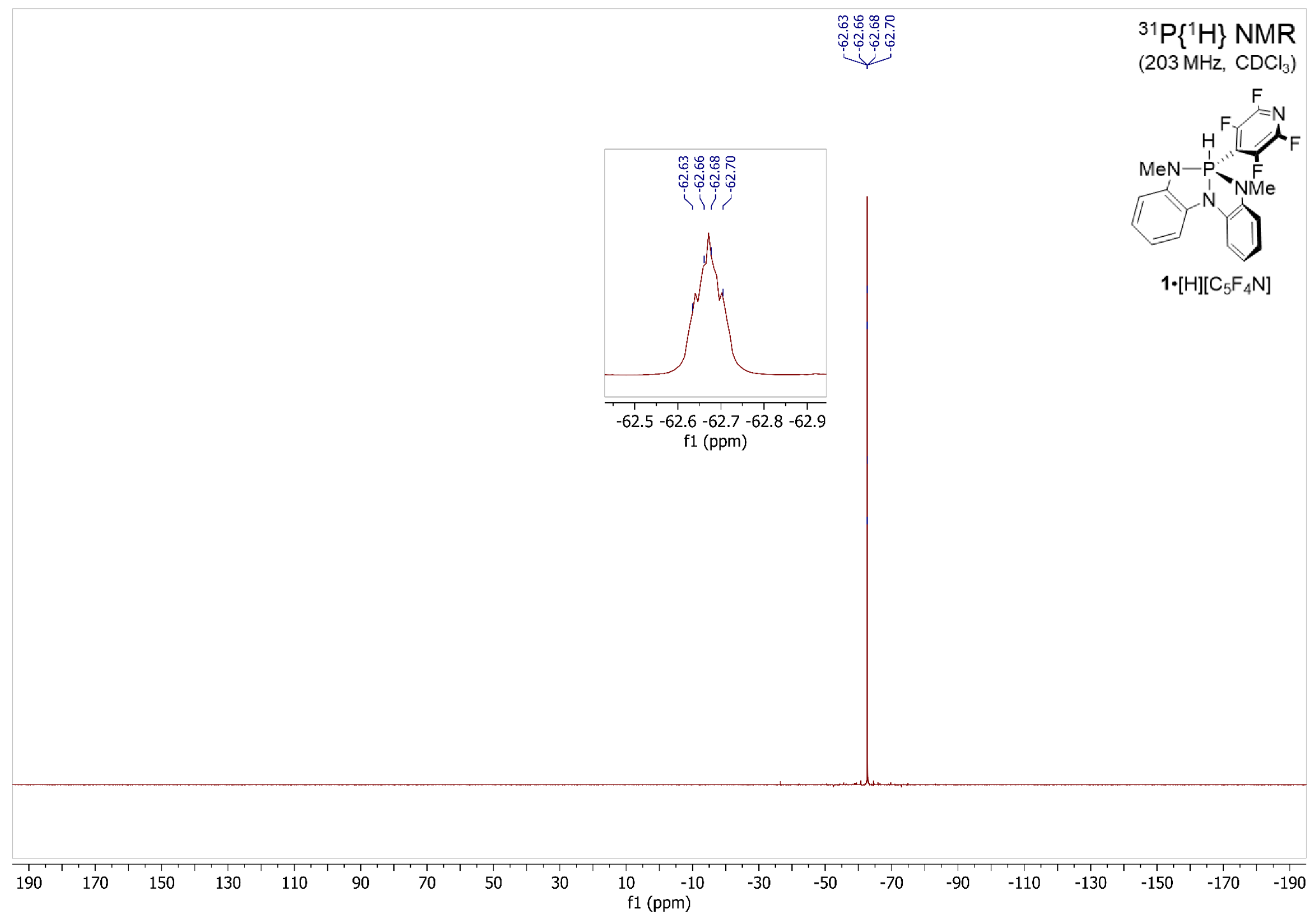




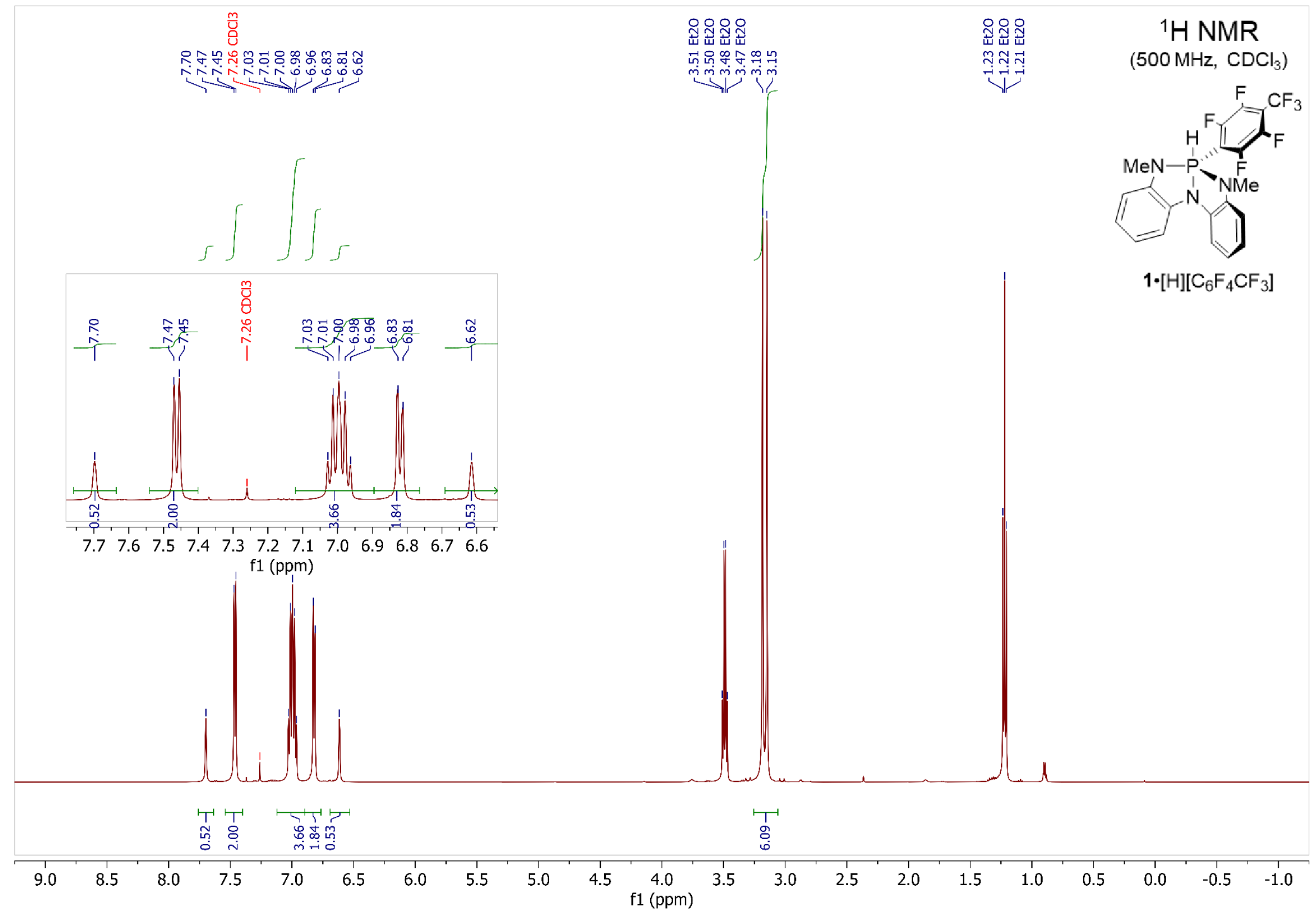




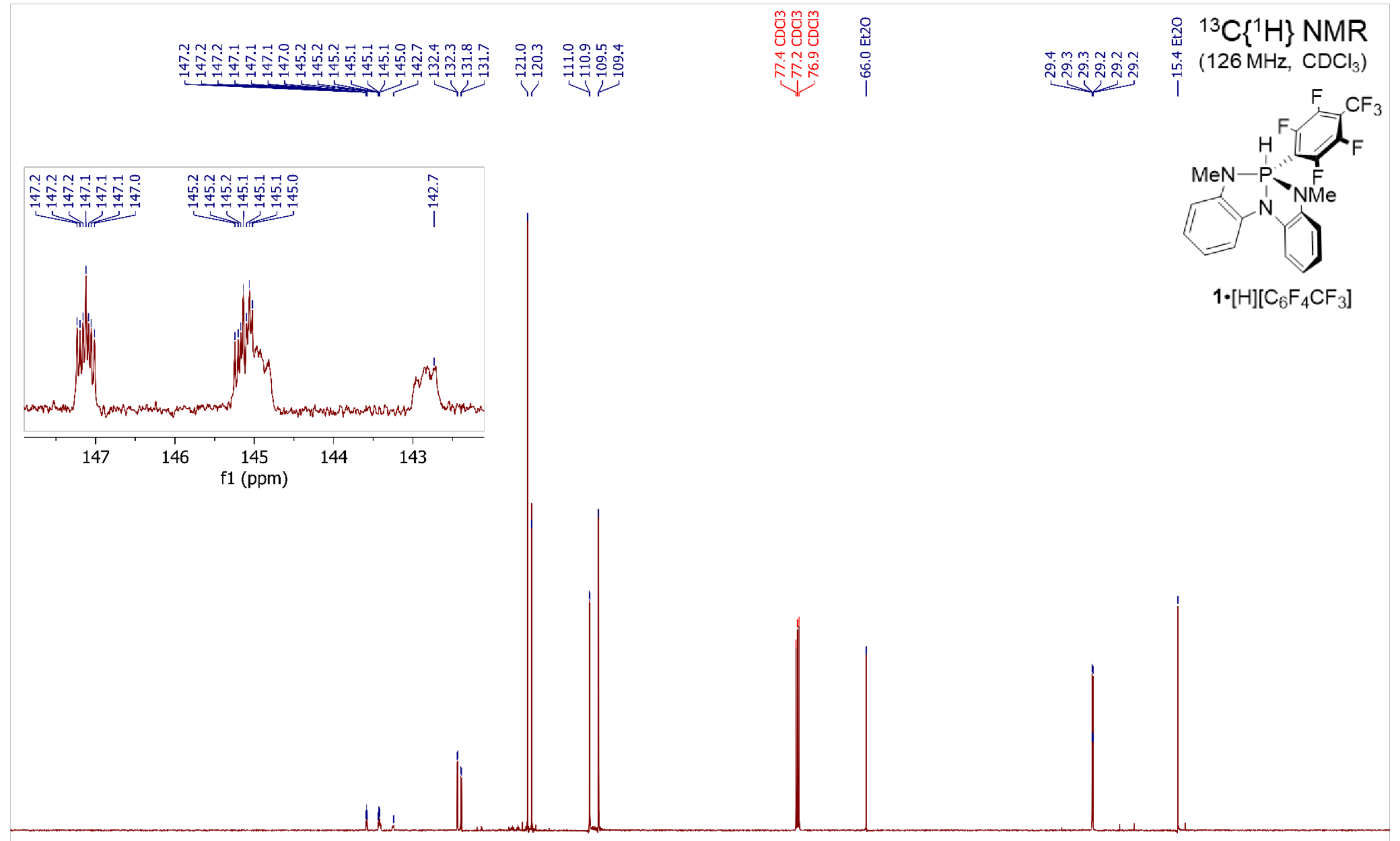

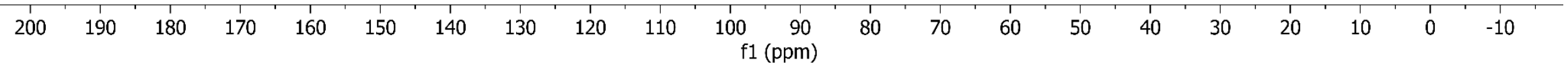




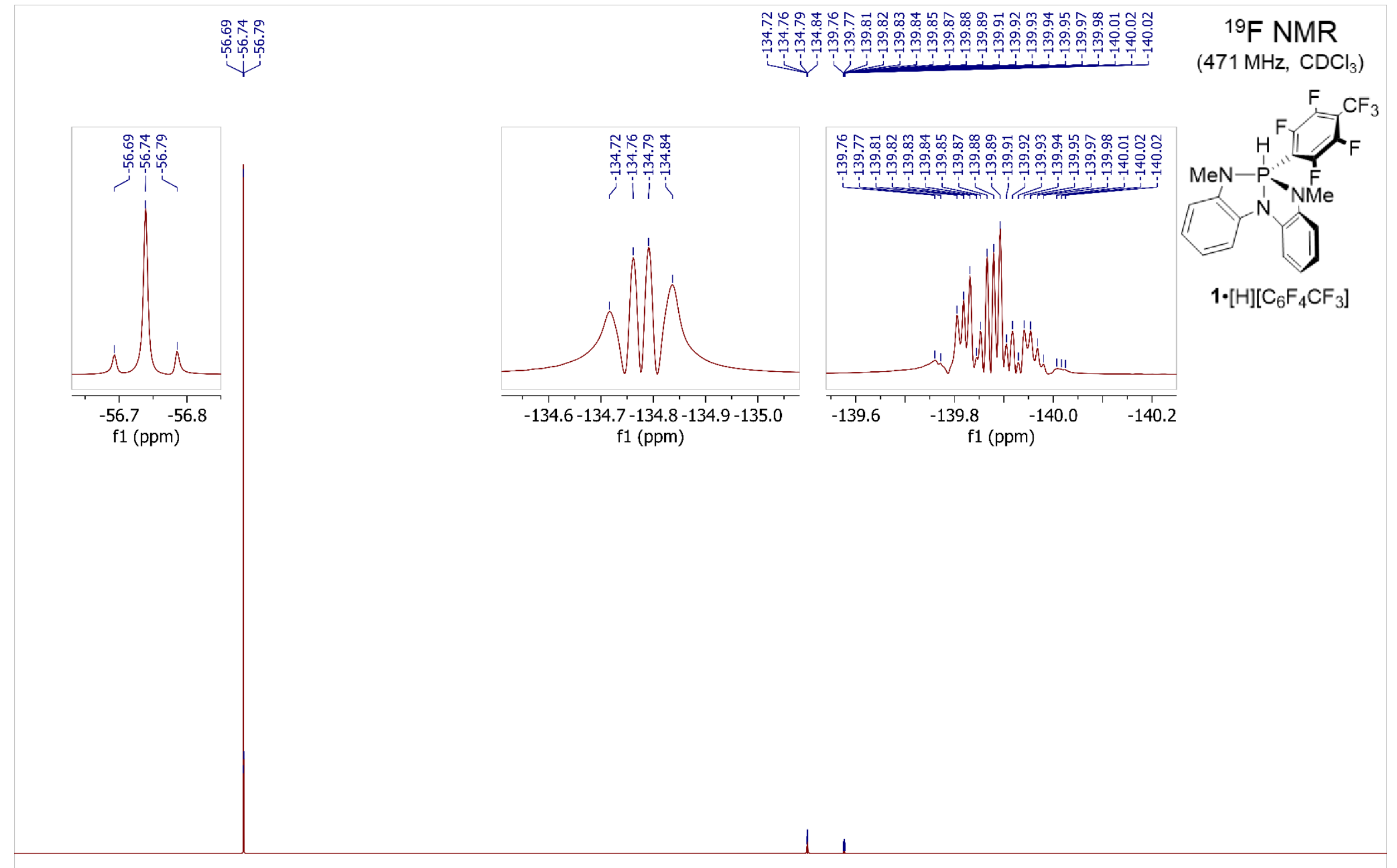

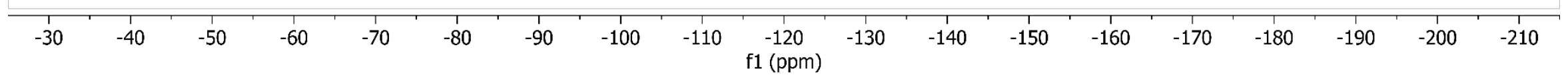




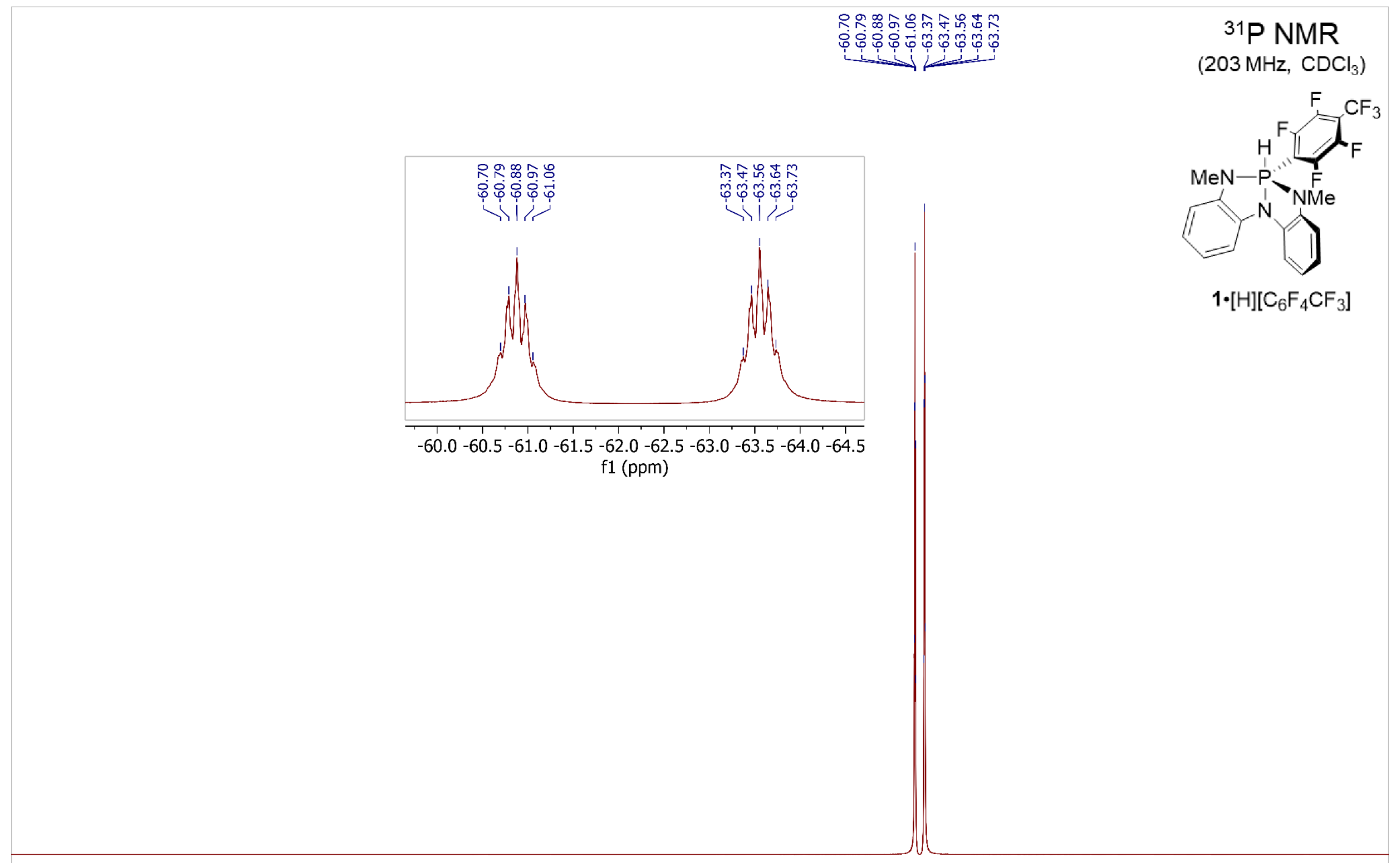

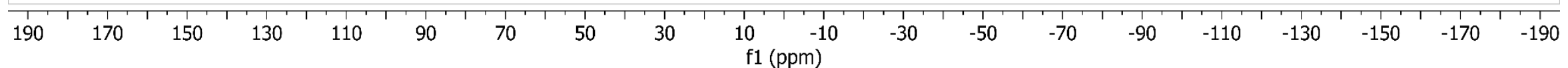




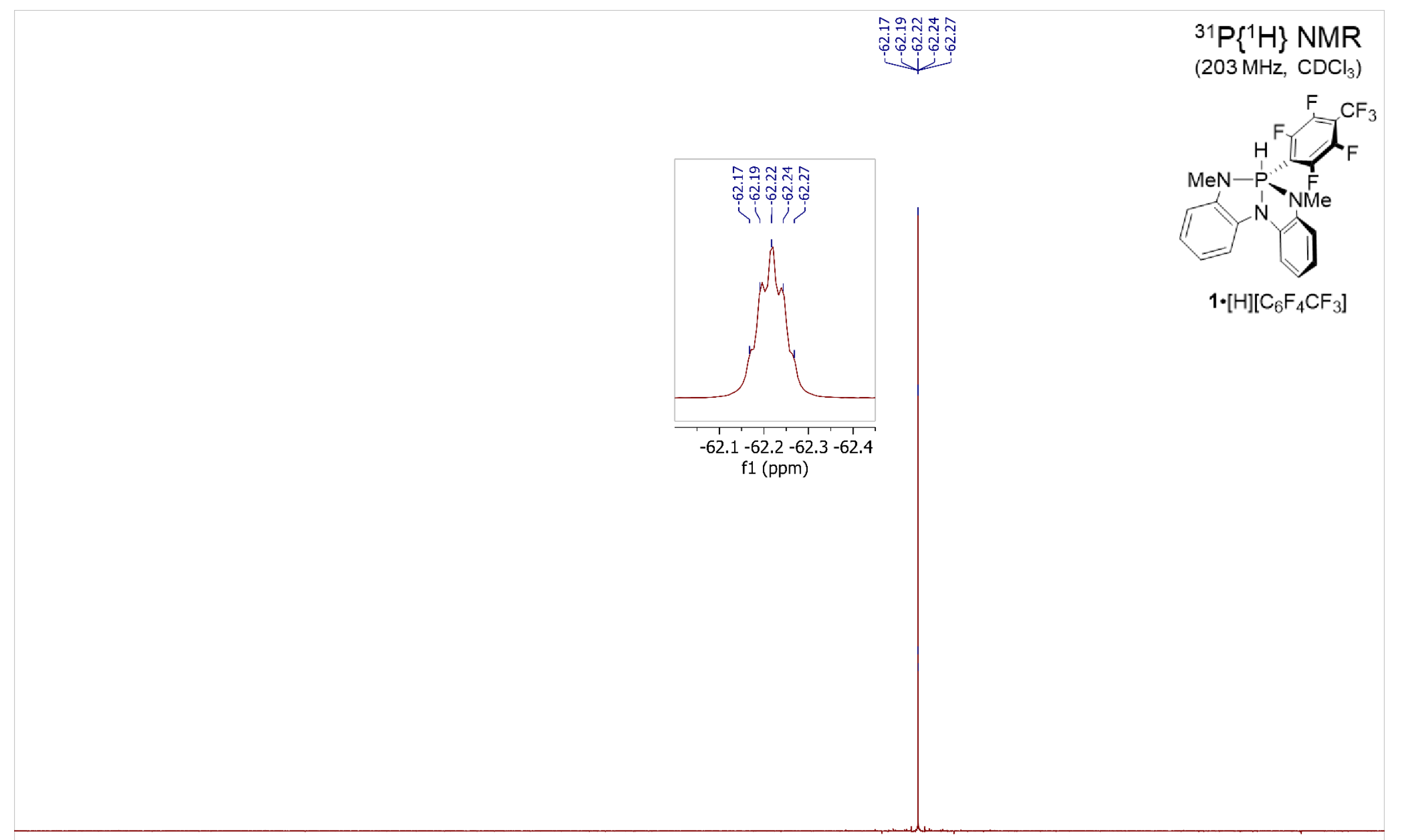

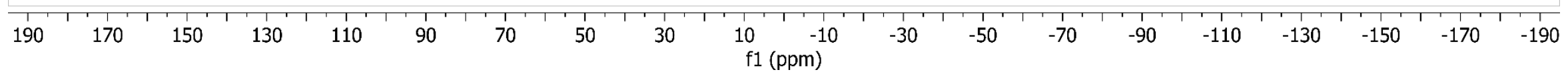




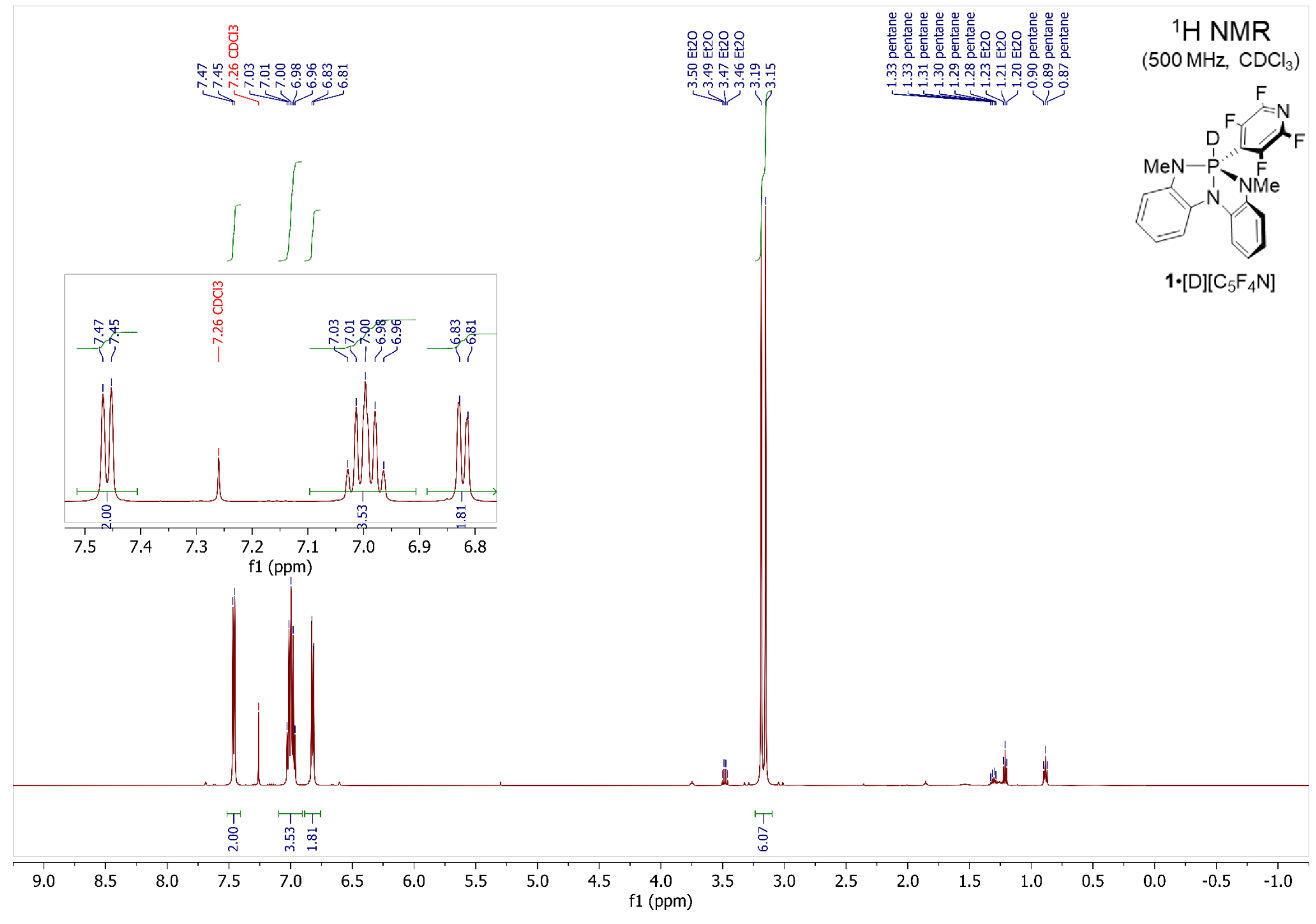



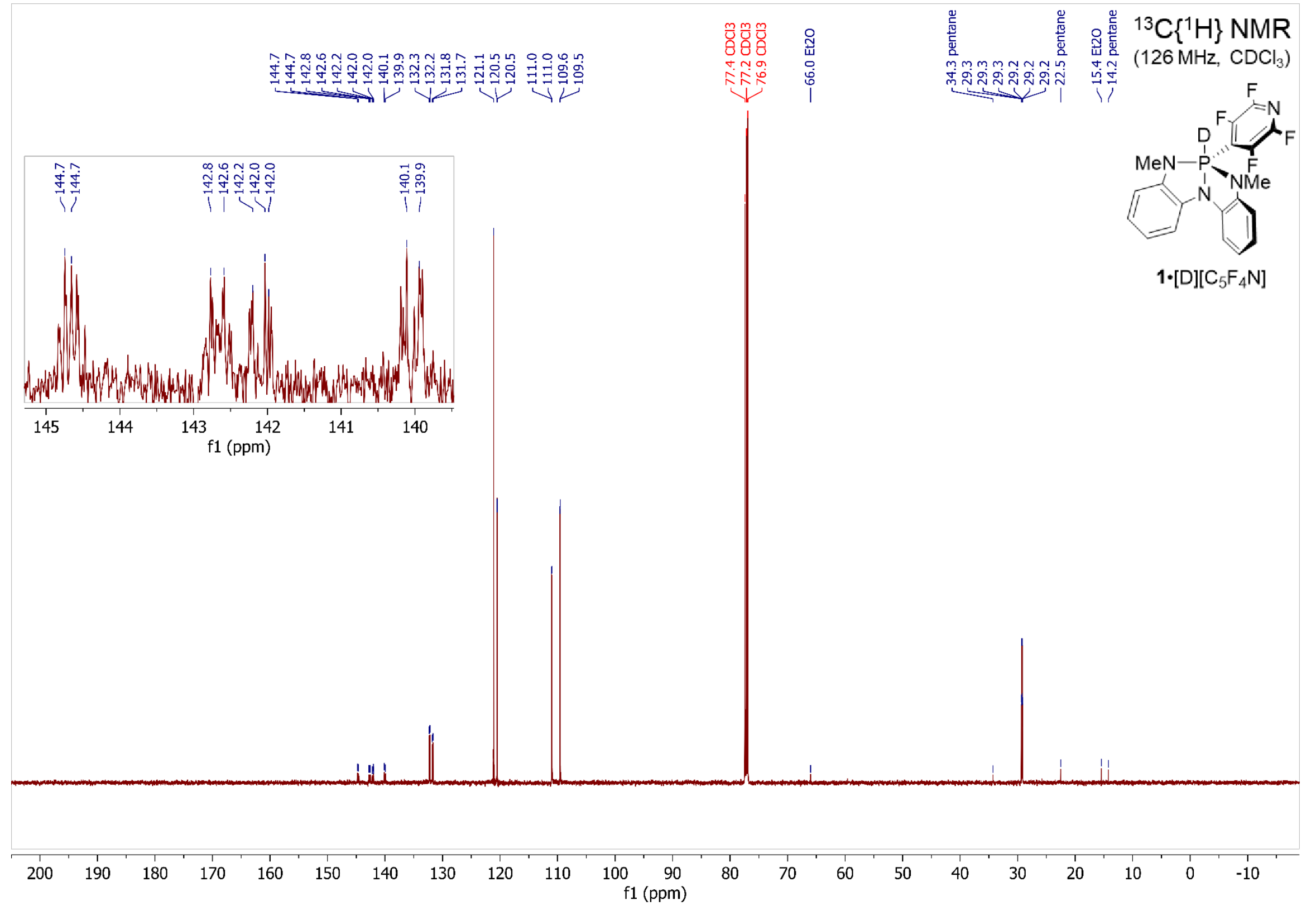


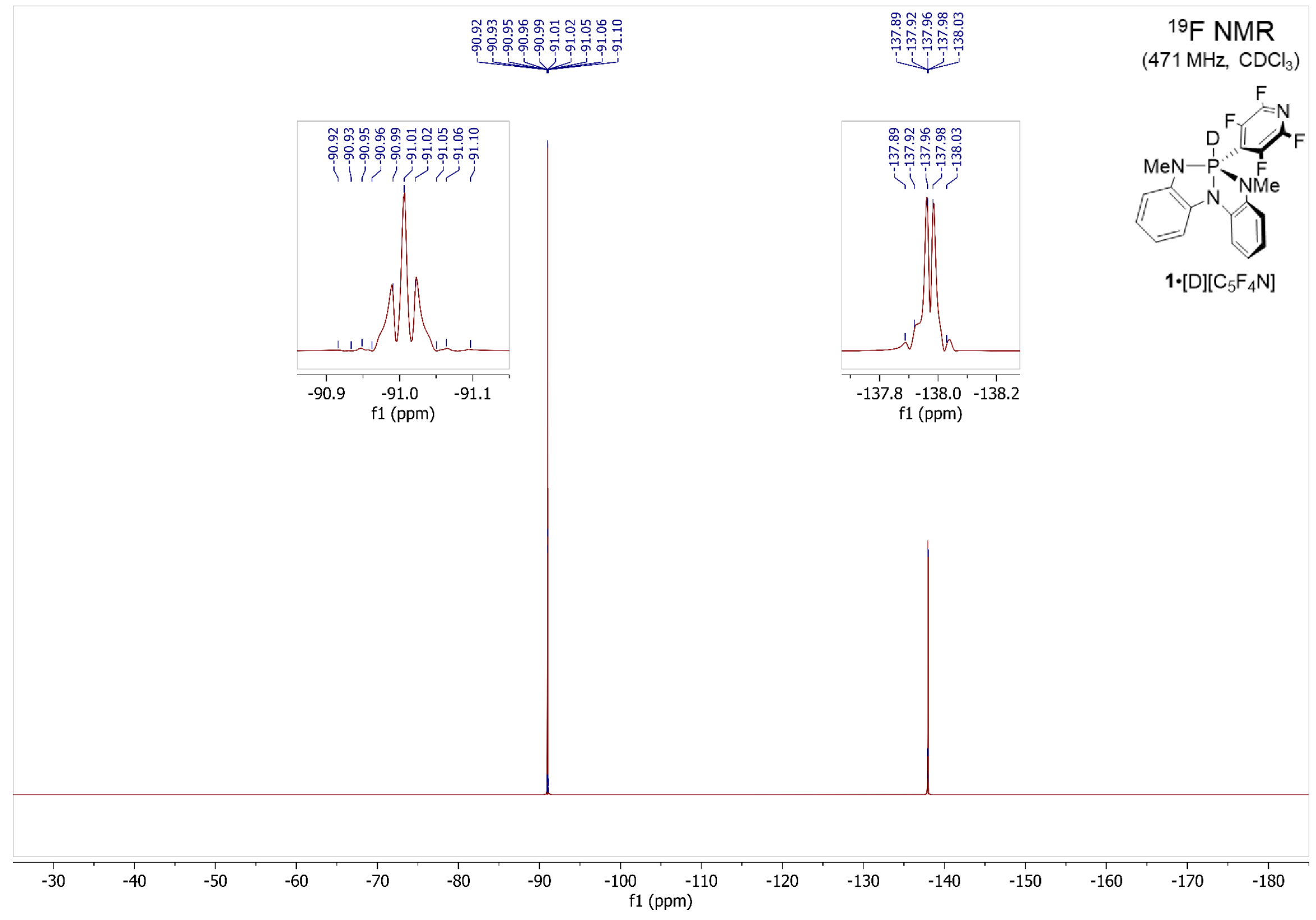




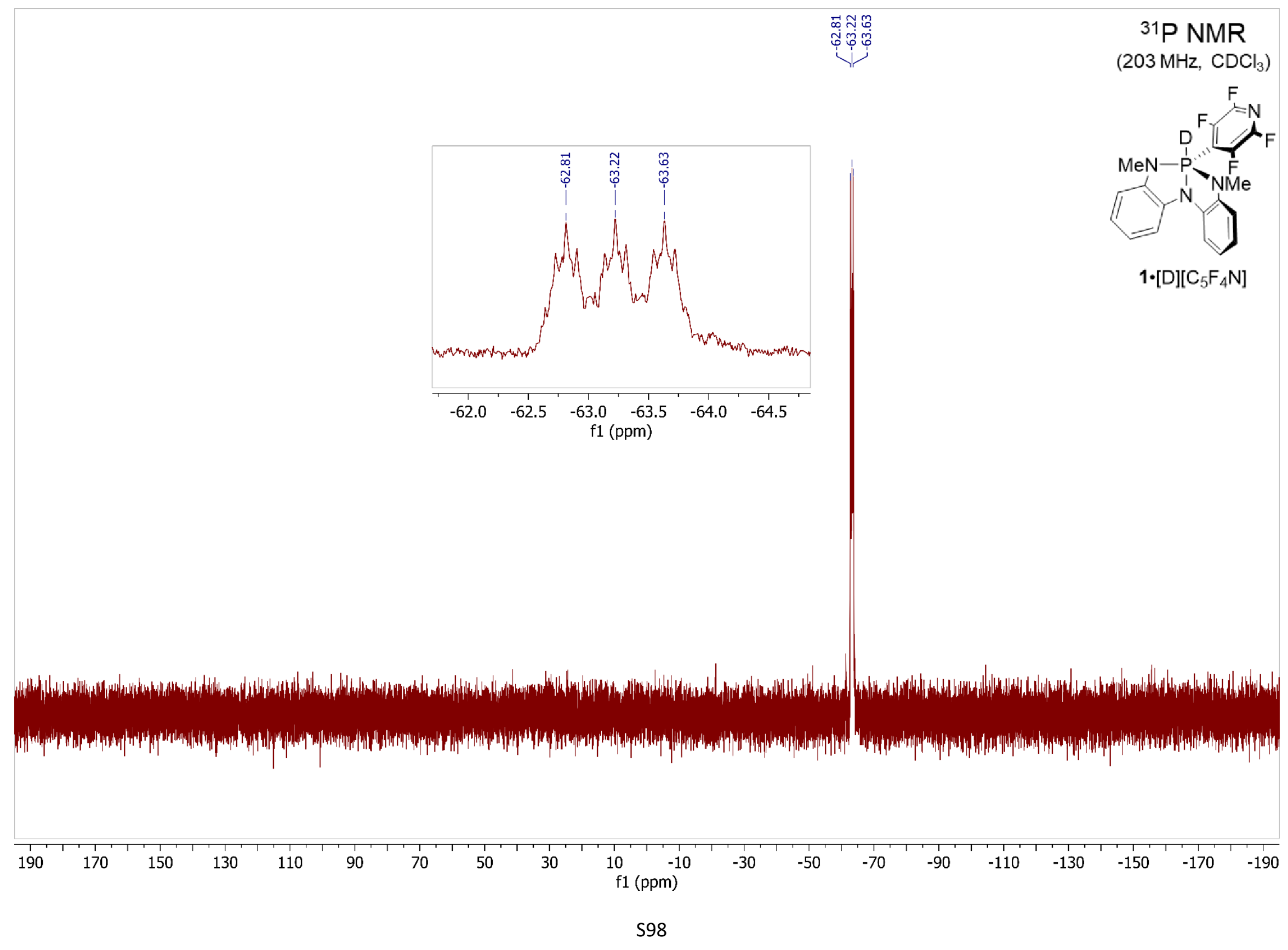




\section{References}

(1) Willcott, M. R. MestRe Nova. J. Am. Chem. Soc. 2009, 131, 13180.

(2) Bruker, SAINT, 2012, Bruker AXS Inc., Madison, Wisconsin, USA.

(3) Bruker, SADABS, 2001, Bruker AXS Inc., Madison, Wisconsin, USA.

(4) Sheldrick, G. M. SHELXT - Integrated space-group and crystal-structure determination. Acta. Cryst. 2015, A71, 3-8.

(5) Zhao, W.; McCarthy, S. M.; Lai, T. Y.; Yennawar, H. P.; Radosevich, A. T. Reversible Intermolecular E-H Oxidative Addition to a Geometrically Deformed and Structurally Dynamic Phosphorous Triamide. J. Am. Chem. Soc. 2014, 136, 17634-17644.

(6) Schrock, R. R.; Lee, J.; Liang, L.-C.; Davis, W. M. The synthesis and structures of tantalum complexes that contain a triamido or a diamidoamine ligand. Inorganica Chim. Acta 1998, 270, 353-362.

(7) Cleveland, G. T.; Radosevich, A. T. A Nontrigonal Tricoordinate Phosphorus Ligand Exhibiting Reversible “Nonspectator” L/X-Switching. Angew. Chem. Int. Ed. 2019, 58, 15005-15009.

(8) Eisch, J. J.; Rhee, S.-G. Kinetic, Stereochemical, and Molecular Association Factors in the Hydralumination of Alkynes. An Inquiry into the Intermediacy of Complexes. J. Am. Chem. Soc. 1974, 96, 7276-7284.

(9) Bochmann, M.; Sarsfield, M. J. Reaction of $\mathrm{AlR}_{3}$ with $\left[\mathrm{CPh}_{3}\right]\left[\mathrm{B}\left(\mathrm{C}_{6} \mathrm{~F}_{5}\right)_{4}\right]$ : Facile Degradation of $\left[\mathrm{B}\left(\mathrm{C}_{6} \mathrm{~F}_{5}\right)_{4}\right]^{-}$by Transient “[ $\left.\mathrm{AlR}_{2}\right]^{+}$”. Organometallics 1998, 17, 5908-5912.

(10) Chen, J.; Chen, E. Y.-X. Unsolvated $\mathrm{Al}\left(\mathrm{C}_{6} \mathrm{~F}_{5}\right)_{3}$ : structural features and electronic interaction with ferrocene. Dalton Trans. 2016, 45, 6105-6110.

(11) Taydakov, I. V. Serious Explosion during Large-Scale Preparation of an Amine by Alane $\left(\mathrm{AlH}_{3}\right)$ Reduction of a Nitrile Bearing a $\mathrm{CF}_{3}$ Group. ACS Chem. Health Saf. 2020, 27 (4), 235-239.

(12) Emsley, J. W.; Lindon, J. C.; Salman, S. R. ${ }^{19} \mathrm{~F}$ Nuclear Magnetic Resonance Spectrum of Pentafluoropyridine Partially Oriented in a Nematic Phase. J. Chem. Soc., Faraday Trans. 2 1972, 68, 1343-1351.

(13) Yow, S.; Gates, S. J.; White, A. J. P.; Crimmin, M. R. Zirconocene Dichloride Catalyzed Hydrodefluorination of $\mathrm{C}_{\mathrm{sp} 2}-\mathrm{F}$ Bonds. Angew. Chem. Int. Ed. 2012, 51 (50), 12559-12563.

(14) Neese, F. The ORCA Program System. WIREs Comput. Mol. Sci. 2012, 2, 73-78.

(15) Chai, J.-D.; Head-Gordon, M. Systematic optimization of long-range corrected hybrid density functionals. J. Chem. Phys. 2008, 128, 084106.

(16) Weigend, F.; Ahlrichs, R. Balanced basis sets of split valence, triple zeta valence and quadruple zeta valence quality for H to Rn: Design and assessment of accuracy. Phys. Chem. Chem. Phys. 2005, 7, 3297-3305. 
(17) NBO 6.0. Glendening, E. D.; Badenhoop, J. K.; Reed, A. E.; Carpenter, J. E.; Bohmann, J. A.; Morales, C. M.; Landis, C. R.; Weinhold, F. Theoretical Chemistry Institute, University of Wisconsin, Madison, 2013.

(18) Glendening, E. D.; Landis, C. R.; Weinhold, F. Natural bond orbital methods. WIREs Comput. Mol. Sci. 2012, 2, 1-42.

(19) Glendening, E. D.; Landis, C. R.; Weinhold, F. NBO 6.0: Natural bond orbital analysis program. J. Comput. Chem. 2013, 34, 1429-1437.

(20) Jmol: an open-source Java viewer for chemical structures in 3D. http://www.jmol.org/

(21) Knizia, G. Intrinsic Atomic Orbitals: An Unbiased Bridge between Quantum Theory and Chemical Concepts. J. Chem. Theory Comput. 2013, 9, 4834-4843.

(22) Knizia, G.; Klein, J. E. M. N. Electron Flow in Reaction Mechanisms-Revealed from First Principles. Angew. Chem. Int. Ed. 2015, 54, 5518-5522.

(23) Rzepa, H. S. KINISOT. A basic program to calculate kinetic isotope effects using normal coordinate analysis of transition state and reactants; http://doi.org/10.5281/zenodo.19272, 2015.

(24) Maeda, S.; Harabuchi Y.; Ono, Y.; Taketsugu, T.; Morokuma, K. Intrinsic reaction coordinate: Calculation, bifurcation, and automated search. Int. J. Quantum Chem. 2015, 115, 258-269. 